\title{
Hadron Structure in Electroweak Precision Measurements
}

\author{
Nathan L. Hall
}

Supervisors: Prof A. W. Thomas and Dr R. D. Young

ARC Centre of Excellence for Particle Physics

at the Terascale and Centre

for the Subatomic Structure of Matter,

Department of Physics,

University of Adelaide

August 1, 2014 



\begin{abstract}
Precision measurements offer important, low-energy tests of the Standard Model. The $Q_{\text {weak }}$ and (proposed) MOLLER experiments at Jefferson Lab are two such measurements. Since the interpretation of the experimental results depends on the precision of the theory prediction, radiative corrections need to be properly accounted for. In this thesis we examine the $\gamma Z$ box correction to the weak charge of the proton. Previously poorly understood, by using phenomenological information to constrain the input structure functions, we determine this important correction at $Q_{\text {weak }}$ kinematics to a precision more than twice that of the previous best estimate. The $\gamma Z$ box is also evaluated at energies relevant to the MOLLER experiment for the first time.

The constructed Adelaide-Jefferson Lab-Manitoba model structure functions may also be used to study other low-energy phenomena. The electromagnetic parametrisations of the cross sections are utilised in the context of the generalised Baldin sum rule to investigate the momentum transfer dependence of the electric and magnetic polarisabilities. Additionally, both the electromagnetic and interference structure functions' moments were calculated in order to determine the higher-twist contributions to the structure functions. These results serve to increase our understanding of the internal structure of the nucleon.
\end{abstract}





\section{Statement of Originality}

I certify that this work contains no material which has been accepted for the award of any other degree or diploma in my name, in any university or other tertiary institution and, to the best of my knowledge and belief, contains no material previously published or written by another person, except where due reference has been made in the text. In addition, I certify that no part of this work will, in the future, be used in a submission in my name, for any other degree or diploma in any university or other tertiary institution without the prior approval of the University of Adelaide and where applicable, any partner institution responsible for the joint-award of this degree.

I give consent to this copy of my thesis, when deposited in the University Library, being made available for loan and photocopying, subject to the provisions of the Copyright Act 1968.

I also give permission for the digital version of my thesis to be made available on the web, via the University's digital research repository, the Library Search and also through web search engines, unless permission has been granted

Nathan L. Hall 



\section{Acknowledgements}

Firstly, thank you to Tony Thomas and Ross Young. If the title of, 'World's Best Supervisors' had been up for grabs, I'm not sure that it would be any longer. Your encouraging feedback, confidence and timely help have made the completion of this thesis not only possible, but rewarding. Thank you to Wally Melnitchouk who has acted like a third, de facto supervisor and whose ideas drove much of this work. Your friendliness and hospitality made visits to Jefferson Lab as comfortable as if I had actually been living there. I would also like to thank Peter Blunden for your efforts in the work presented here and for offering a next step. Thank you also to Fred Myhrer and Will Detmold for allowing me to come and visit.

To my officemates Manuel, Phiala and (more recently) Ryan, thank you for your friendship, for hang-out times and for being willing to help and offer advice even when you had plenty of your own work to do. Phi, your drive and energy are simply amazing. Manuel, your hard work and perseverance are inspiring. Ryan, one day I may actually be able to answer some of your questions. To Ben, if there had been another desk in the office you would have easily fitted in. Thanks for the chats, lunches and help with the formatting and write-up of this thesis. Daniel, Adrian and Alex what am I going to do in Manitoba without the excuse of a football tournament to have gelati?

Dad and Mum, thank you for your belief, unwavering support and for allowing me complete freedom in pursuing this path. Jason, Josiah and Becky, thank you for your company outside of university life and for providing an outlet to take my mind off things. To everyone at Westbourne Park Uniting Church, and especially to members of my (past and present) small group, thanks for hanging in there with me-I'm finally finished! John Blanksby, thank you for your prayers and for providing a listening ear when I needed it.

Finally, God, thank you. Your mercies have been new every morning and your faithfulness has indeed been great. 



\section{Contents}

1 Introduction 1

2 The Standard Model and beyond 5

2.1 The Standard Model . . . . . . . . . . . . . . . 5

2.2 Electroweak theory . . . . . . . . . . . . . . . . . . 6

2.3 Beyond the SM . . . . . . . . . . . . . . 9

3 Precision tests of the SM 17

3.1 Atomic parity violation . . . . . . . . . . . . . 17

3.2 Parity-violating electron scattering . . . . . . . . . . . . . . . . . . . . . . .

3.3 Radiative corrections to $Q_{W}^{p} \ldots \ldots \ldots 20$

3.4 The $\square_{\gamma Z}$ correction . . . . . . . . . . . . . . . . 22

$\begin{array}{llr}4 & \text { Structure functions } & 27\end{array}$

4.1 Deep inelastic scattering . . . . . . . . . . . . . . . . . 29

4.2 Quark-parton model . . . . . . . . . . . . . . . . 32

4.3 Modelling structure functions . . . . . . . . . . . . . . . 33

$4.4 \gamma Z$ interference structure functions . . . . . . . . . . . 38

5 Adelaide-Jefferson Lab-Manitoba model 43

5.1 Electromagnetic parametrisation . . . . . . . . . . . . . 43

5.2 AJM $\gamma Z$ interference structure functions . . . . . . . . . . . . 48

5.3 Phenomenological constraints . . . . . . . . . . . 50

$6 \quad$ The $\square_{\gamma Z}^{V}$ correction $\quad 65$

$6.1 \gamma Z$ box corrections for $Q_{\text {weak }} \ldots \ldots$. . . . . . . 66

6.2 Predictions for parity-violating asymmetries . . . . . . . . . 69

$6.3 Q_{W}^{p}$ at $11 \mathrm{GeV} \ldots \ldots \ldots . \ldots . \ldots 72$

$6.4 A_{\text {PVDIS }}$ for the proton at $11 \mathrm{GeV} \ldots \ldots . \ldots . \ldots 8$

7 Electric and magnetic polarisabilities of the proton $\quad 85$

7.1 The generalised Baldin sum rule . . . . . . . . . . . . . 85

$7.2 Q^{2}$ dependence of $\alpha$ and $\beta \ldots \ldots . \ldots . \ldots 8$ 
8 Quark-hadron duality $\quad 95$

8.1 Duality in the SM . . . . . . . . . . . . . . . . 95

8.2 Moments of structure functions . . . . . . . . . . . . . . 97

8.3 Implications for the $\square_{\gamma Z}^{V}$ correction . . . . . . . . . . . . 103

9 Summary and conclusion $\quad 105$

$\begin{array}{ll}\text { A Further determinations of } \Re e \square_{\gamma Z}^{V} & 109\end{array}$

$\begin{array}{ll}\text { B List of publications } & 111\end{array}$

$\begin{array}{lr}\text { Bibliography } & 113\end{array}$ 


\section{Introduction}

The Standard Model (SM) of particle physics currently provides the best account of the properties and interactions of the elementary particles which make up matter. It combines quantum chromodynamics (QCD), which governs the strong force between quarks and gluons inside the nucleon, with electroweak theory - the unified theory of weak and electromagnetic interactions. Since its inception, the SM has enjoyed an exceptional degree of success, accurately explaining and predicting a wide range of experimental phenomena. Nevertheless, a growing body of evidence suggests that it is in fact, a low-energy, effective theory of a deeper, underlying description.

Because of the fundamental importance of the SM, new experiments are continually being designed which extend current empirical limits - with respect to either the energy scale, the precision level, or both-in order to test further the agreement between theory and nature. Experiments such as those being performed at the Large Hadron Collider (LHC) in CERN study particle collisions at energies never previously achieved, whereas parity-violating (PV) measurements like $Q_{\text {weak }}$ provide precision checks of SM observables. In this thesis we are specifically interested in low-energy precision tests of the SM.

In 2012, the $Q_{\text {weak }}$ collaboration completed the data collection stage of their PV experiment measuring the weak charge of the proton - the weak force's analogue of the electric charge. An early analysis that included four percent of the total data revealed good agreement with the SM prediction. Results for the full data set are expected later this year. The aim, ultimately, is to determine the proton's weak charge, $Q_{W}^{p}$, to a precision of $4 \%$. This is equivalent to measuring the weak mixing angle, $\sin ^{2} \theta_{W}$, to $0.3 \%$ and would give the most precise determination of $\sin ^{2} \theta_{W}$ away from the $Z$-pole. To achieve such a goal, radiative corrections to the theory value of $Q_{W}^{p}$ must be reliably estimated and their uncertainties fully understood.

Although most of the radiative corrections to the proton's weak charge are indeed well understood, the $\gamma Z$ 'box' diagram, arising from the interference between the photon and $Z$-boson exchanges, remains subject to considerable debate. Much of this controversy stems from a lack of empirical data on the interference structure functions - used as inputs in the dispersion calculation. An accurate experimental knowledge of the interference structure functions 
would remove any significant doubt in the $\gamma Z$ box determination. However, as it currently stands, various models of the structure functions are employed. It is therefore necessary to quantify and minimise of the uncertainties resulting from these models. In constructing the Adelaide-Jefferson Lab-Manitoba (AJM) model - presented in this work - we make use of the excellent understanding of parton distribution functions (PDFs) to constrain the input $\gamma Z$ structure functions. This is the first time this has been done in the literature, all previous work having relied primarily on the accuracy of the models themselves to obtain a good estimate of the uncertainty.

Since this work is done in the context of the SM, in Chapter 2 we begin with a brief overview of the theory. An introduction to electroweak theory and a more detailed discussion of PV in the SM will also be included in this chapter. As one of the principal aims of the $Q_{\text {weak }}$ experiment is to search for physics beyond the Standard Model (BSM), in Section 2.3 we present some of the main reasons why many physicists expect that there may be a more fundamental theory. This is followed by a listing of some of the leading candidates for new physics (NP).

Most of the results based on the AJM model apply directly to the $Q_{\text {weak }}$ experiment. There are, however, a number of other relevant low-energy measurements. The E08-011 experiment - another PV experiment at Jefferson Lab-measured the asymmetry which arises in electron-deuteron scattering and its results will be used as an important check of the AJM model methodology. Additionally, the proposed MOLLER experiment contains backgrounds which we may estimate using the AJM structure functions. The details of these experiments are included in Section 3.2, where we have also included a discussion on past measurements and planned, future experiments. Following this, Section 3.3 examines the radiative corrections to $Q_{W}^{p}$, while the final section of Chapter 3 looks specifically at the $\square_{\gamma Z}$ correction. The dispersion formalism needed for the calculation will be introduced here.

It will become evident that the most important inputs in the $\square_{\gamma Z}$ calculation are the interference structure functions and as a result, the entire Chapter 4 is devoted to these objects. Here we investigate the models of the electromagnetic and $\gamma Z$ structure functions that have been used in the literature. Since we are not only interested in the central values of the $\gamma Z$ box obtained by the different groups but would also like to know how the uncertainties arise, we will include brief error analyses of the various models. Additional attention will be given to the model which has the largest errors.

Having completed the review of earlier work, the next chapter presents the Adelaide-Jefferson Lab-Manitoba model. The construction of the interference structure functions begins with the electromagnetic parametrisations, which we introduce in Section 5.1. A description of the $\gamma Z$ structure function follows, along with a detail explanation of the method used to constrain the structure functions. Although the constraints provided by the parton distributions should suffice, it would be useful to check our approach empirically. As mentioned previously, this is possible using the data from the E08-011 ex- 
periment and therefore, in the last part of Chapter 5, we test the AJM model asymmetry predictions against these experimental results.

The determination of the vector hadron part of the $\square_{\gamma Z}$ correction using the AJM model is laid out in Chapter 6. Since the hadronic axial-vector contribution has been calculated to sufficient precision previously, we concentrate largely on the $\square_{\gamma Z}^{V}$ term. A small exception to this occurs in the results on the MOLLER backgrounds where the $\square_{\gamma Z}^{A}$ value must be included in the total $\gamma Z$ box correction. Section 6.1 gives the results for the $\square_{\gamma Z}^{V}$ term relevant for $Q_{\text {weak }}$ energies, while Section 6.3 examines the contribution at the higher energies associated with the MOLLER experiment. In between, we make further comparisons of the AJM model asymmetry predictions with those values recently released by the E08-011 experiment. These new results differ from the previous ones in that the kinematics they examine are no longer in the nucleon resonance region, but the deep inelastic scattering (DIS) region. An evaluation of the inelastic proton asymmetry - one of the significant backgrounds in the MOLLER experiment-concludes this chapter.

Although the primary motivation for the AJM model comes from the need to accurately determine the $\square_{\gamma Z}^{V}$ contribution, the uses for these structure functions are much more wide-reaching. In the rest of this thesis we address some of these additional applications.

In Chapter 7 we utilise the $F_{1}$ electromagnetic structure function to calculate the momentum transfer $\left(Q^{2}\right)$ dependence of the proton's electric and magnetic polarisabilities. Following a brief discussion of earlier work, the generalised Baldin sum rule is used to show the $Q^{2}$-evolution of the polarisabilites down to much lower momentum than previously attained. Several experimental efforts have been involved in studying these observables and a comparison (Section 7.2) between the data and the AJM parametrisation shows good agreement between the two, re-emphasising the reliability of our construction.

Quark-hadron duality is the observation that the averaged resonance region structure functions closely resemble those given by partonic description. Although intuitive to some degree, the level of agreement and the kinematic range over which it occurs is surprising. Furthermore, the theoretical framework used to describe this phenomena, the operator product expansion (OPE), fails to explain physically how the transition from resonances to scaling takes place. One of the principal ways of studying duality is through the structure function moments. In Chapter 8 we include a summary of the OPE and the necessary formalism before considering the moments of the electromagnetic and interference $F_{2}$ structure functions.

Our motivation for studying duality is twofold: firstly, a comparison of the total structure function moments with the leading-twist moments allows one to extract the higher-twist contributions to the structure functions. Secondly, should we observe duality in the $\gamma Z$ structure functions moments, it will help confirm the estimates of the $\square_{\gamma Z}^{V}$ correction obtained earlier. Section 8.3 includes AJM model results for the $M_{2}$ moment, where we also show the calculations of the neutron moments. Although these incorporate larger 
errors, they are important when considering higher-twist contributions to the electron-deuteron asymmetries.

The final chapter of this thesis summarises the discussion of the preceding chapters, while also revisiting some of the main conclusions. Further avenues for extending the work presented here are also included. 


\section{The Standard Model and beyond}

The $Q_{\text {weak }}$ experiment is a precision test of the Standard Model. In this chapter we give an introduction to the SM, concentrating particularly on electroweak theory. Problems with the SM are reviewed, which leads naturally into a discussion on possible BSM physics and their experimental searches.

\subsection{The Standard Model}

Based on the $S U(3)_{c} \times S U(2)_{L} \times U(1)_{Y}$ gauge group, the SM successfully predicts the behaviour of three out of the four observed forces. The only one not included in this description is gravity. The strong interactions are a result of $S U(3)$ colour (hence ' $c$ ') symmetry, whereas the unified electromagnetic and weak interactions are accounted for by the $S U(2)_{L} \times U(1)_{Y}$ factor. The subscript $L$ refers to the fact that the $S U(2)$ part acts only on left-handed fermions, while the $Y$ factor is the weak hypercharge.

Interactions between the leptons and quarks which make up nuclear matter occur via the exchange of vector bosons. In the case of QCD, massless gluons are responsible for the strong force experienced by quarks, while the weak force is mediated by the massive $W^{ \pm}$and $Z^{0}$ bosons. The photon completes the force-carrier sector and mediates the familiar electromagnetic interactions.

Both leptons and quarks may be divided into three generations according to their mass and electric charge (cf. Table 2.1). Quarks, under the influence of the strong force, combine to form mesons (quark-antiquark pairs) and baryons (three quark systems). These hadrons in turn, form multiplets, whose structure is largely - since $S U(3)$ flavour is not exact - governed by the irreducible representations of $S U(3)$. The leptons on the other hand, experience only the weak and electromagnetic interactions. Completing the particle content of the SM is the recently discovered $[1,2]$ Higgs boson associated with the Higgs field, responsible for generating mass in the SM.

From these fundamental building blocks the rest of nuclear matter in 
Table 2.1: Properties of the three generations of SM matter particles. The data is taken from the Particle Data Group [3].

\begin{tabular}{cccc|ccc}
\hline \hline Gen. & Quark & Charge & Mass $(\mathrm{MeV})$ & Lepton & Charge & Mass $(\mathrm{MeV})$ \\
\hline I & $u$ & $2 / 3$ & $1.8-3.0$ & $e$ & -1 & 0.511 \\
& $d$ & $-1 / 3$ & $4.5-5.3$ & $\nu_{e}$ & 0 & $<2 \times 10^{-6}$ \\
\hline II & $s$ & $-1 / 3$ & $90-100$ & $\mu$ & -1 & 106 \\
& $c$ & $2 / 3$ & $1.28 \times 10^{3}$ & $\nu_{\mu}$ & 0 & $<0.19$ \\
\hline \multirow{2}{*}{ III } & $t$ & $2 / 3$ & $1.73 \times 10^{5}$ & $\tau$ & -1 & $1.78 \times 10^{3}$ \\
& $b$ & $-1 / 3$ & $4.18 \times 10^{3}$ & $\nu_{\tau}$ & 0 & $<18.2$ \\
\hline \hline
\end{tabular}

the universe is presently understood to be constructed.

\section{$2.2 \quad$ Electroweak theory}

The theoretical footings for the unification of the SM electroweak theory were first introduced by Glashow [4] in 1961. The massless vector bosons associated with this $S U(2) \times U(1)$ gauge symmetry are $W_{\mu}^{i}$ (where $\left.i=1,2,3\right)$ and $B_{\mu}$, whilst the couplings are $g$ and $g^{\prime}$. These gauge bosons are included in the SM in the kinetic terms of the Lagrangian,

$$
\mathcal{L}=-\frac{1}{4} W_{\mu \nu}^{i} W^{\mu \nu i}-\frac{1}{4} B_{\mu \nu} B^{\mu \nu},
$$

where the field strength tensors are,

$$
\begin{aligned}
W_{\mu \nu}^{i} & =\partial_{\mu} W_{\nu}^{i}-\partial_{\nu} W_{\mu}^{i}-g \epsilon_{i j k} W_{\mu}^{j} W_{\nu}^{k}, \quad i, j, k=1,2,3 ; \\
B_{\mu \nu} & =\partial_{\mu} B_{\nu}-\partial_{\nu} B_{\mu} .
\end{aligned}
$$

Under $S U(2)$ 'weak isospin' the left-handed fermions transform as doublets. The $U(1)$ gauge group acts on both left- and right-handed components of the fermion fields and is associated with a phase symmetry [5]. In order to generate the masses of the physical particles, the $S U(2) \times U(1)$ symmetry is spontaneously broken $[6,7]$ when minimising a potential,

$$
V(\phi)=\mu^{2} \phi^{\dagger} \phi+\frac{\lambda^{2}}{2}\left(\phi^{\dagger} \phi\right)^{2},
$$

involving a complex scalar doublet,

$$
\phi=\left(\begin{array}{c}
\phi^{+} \\
\phi^{0}
\end{array}\right) .
$$

This is known as the Higgs mechanism [8, 9, 10, 11].

After spontaneous symmetry breaking, the $U(1)_{Q}$ group is the only remaining unbroken symmetry. Additionally, the weak mediators end up acquiring mass leaving the photon as the sole massless vector boson. Quantum 
electrodynamics (QED) is based on this $U(1)_{Q}$ group. Proof of the renormalisability of such spontaneously broken gauge theories by t'Hooft and Veltman $[12,13,14]$ in the early 1970s completed the theoretical development of electroweak theory. Experimentally, however, there still remained some puzzling issues.

\section{Parity violation in EW theory}

In 1956 Lee and Yang [15] put forward the idea of parity non-conservation in weak interactions. An exceptionally radical thought at the time, this was soon verified experimentally by $\mathrm{Wu}$ et al. [16] and confirmed by a group lead by Lederman [17]. Both of these experiments involved charged current reactions; however, Glashow's theory also predicted a small amount of parity violation through the neutral current (NC) interaction term

$$
J_{i n t}^{\mu}=\frac{g}{2 \cos \theta_{W}} J_{\mathrm{NC}}^{\mu}(x) Z_{\mu}(x)
$$

where,

$$
J_{\mathrm{NC}}^{\mu}(x)=\sum_{i} \bar{\psi}_{i}(x) \gamma^{\mu}\left(g_{V}^{i}-g_{A}^{i} \gamma^{5}\right) \psi_{i}(x)
$$

and $Z_{\mu}(x)$ is the $Z^{0}$-boson field. The fermion (anti-fermion) fields are given by $\psi_{i}\left(\bar{\psi}_{i}\right)$, and the vector and axial-vector couplings,

$$
\begin{aligned}
g_{V}^{i} & \equiv t_{3 L}^{i}-2 q_{i} \sin ^{2} \theta_{W} \\
g_{A}^{i} & \equiv t_{3 L}^{i}
\end{aligned}
$$

where $t_{3 L}^{i}$ is the weak isospin of the fermion $i$ and $q_{i}$ the charge.

After several conflicting experimental results $[18,19,20]$, PV in neutral currents was finally confirmed by Prescott et al. [21] in 1978 using electrondeuterium scattering and reaffirmed by a second experiment the following year [22]. The measured asymmetries are shown in Fig. 2.1. Together with the indirect $[23,24,25]$ and direct $[26,27]$ observations of the $Z^{0}$-boson at CERN, these results firmly established the $S U(2) \times U(1)$ theory as the principal description of the unified weak and electromagnetic interactions.

Since then the theory has proven to be extremely robust, successfully predicting the values of physical observables to unprecedented levels of precision. Advancements in technologies has meant that it is now possible to search for deviation between experiments and theory at the parts per billion (ppb) level. In Sections 3.1 and 3.2 we will discuss these developments in more detail. 

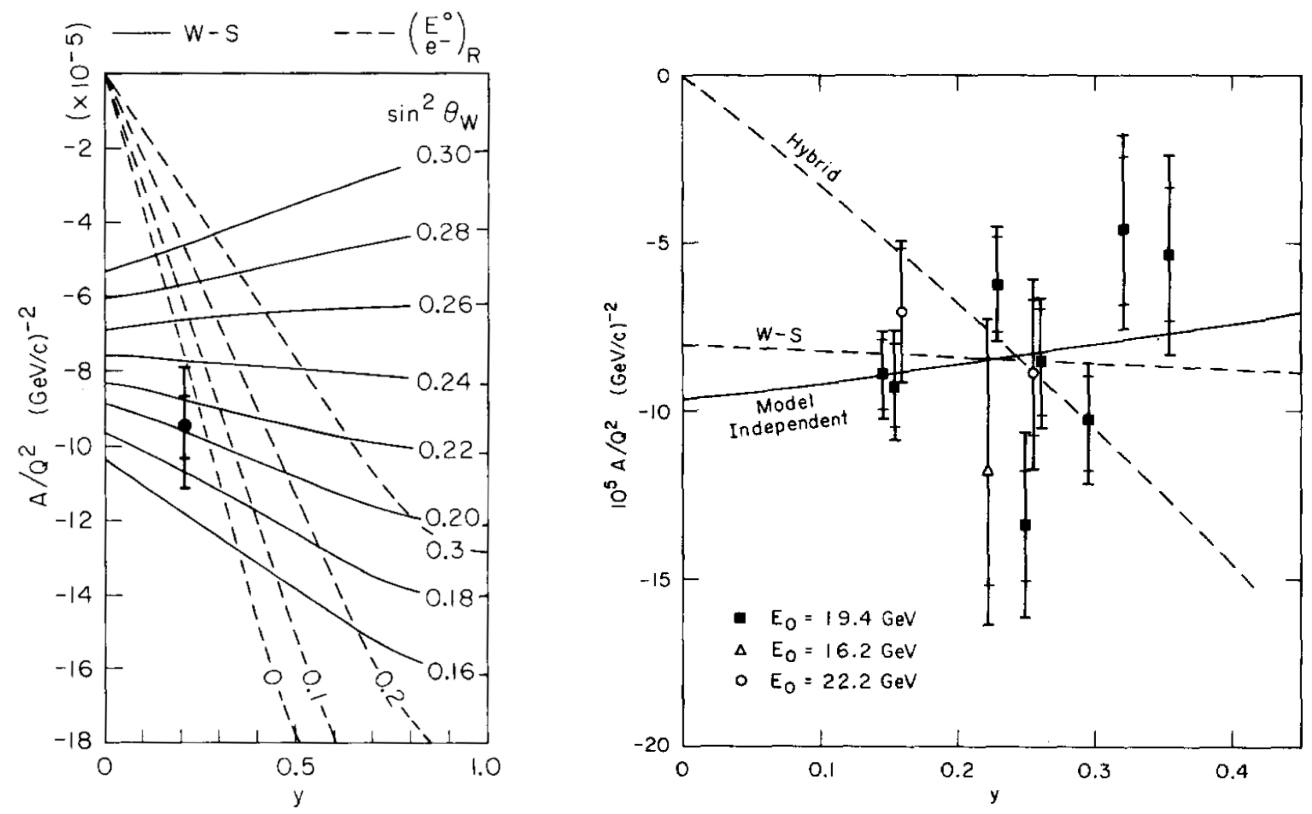

Figure 2.1: Parity violation in $e-d$ scattering as observed by Prescott et al. $[21,22]$. On the left-hand plot, the dashed line represents the same 'Hybrid' model shown in the right-hand figure. Both asymmetries $(A)$ are normalised by the momentum transfer $\left(Q^{2}\right)$ and plotted against the fraction of energy lost by the incoming electron $(y)$.

\section{The weak mixing angle}

Mixing of the four vector fields, $W_{\mu}^{i}$ and $B_{\mu}$, gives,

$$
\begin{aligned}
W_{\mu}^{ \pm} & =\frac{1}{\sqrt{2}}\left(W_{\mu}^{1} \mp W_{\mu}^{2}\right), \\
Z_{\mu} & =\frac{1}{\sqrt{g^{2}+g^{\prime 2}}}\left(g W_{\mu}^{3}-g^{\prime} B_{\mu}\right), \\
A_{\mu} & =\frac{1}{\sqrt{g^{2}+g^{\prime 2}}}\left(g^{\prime} W_{\mu}^{3}+g B_{\mu}\right),
\end{aligned}
$$

where the $W_{\mu}^{ \pm}$and $Z_{\mu}$ are the mediator fields of the weak interactions and $A_{\mu}$ is the photon field of the electromagnetic force.

Alternatively, one can define the weak mixing angle (also referred to as the Weinberg angle) in terms of the coupling constants,

$$
\sin \theta_{W}=\frac{g^{\prime}}{\sqrt{g^{2}+g^{\prime 2}}}, \quad \cos \theta_{W}=\frac{g}{\sqrt{g^{2}+g^{\prime 2}}},
$$

such that,

$$
\begin{aligned}
& Z_{\mu}=\cos \theta_{W} W_{\mu}^{3}-\sin \theta_{W} B_{\mu}, \\
& A_{\mu}=\sin \theta_{W} W_{\mu}^{3}+\cos \theta_{W} B_{\mu} .
\end{aligned}
$$


In order to verify experimentally, a renormalised definition of $\sin ^{2} \theta_{W}$ is required. At lowest order in perturbation theory,

$$
\sin ^{2} \theta_{W}=1-\frac{M_{W}^{2}}{M_{Z}^{2}}
$$

and this expression was used extensively earlier on. However, it also induced misleading radiative corrections involving the top quark mass which meant that an alternative definition was needed [28].

Today, the most commonly used definition of the weak mixing angle comes from the modified minimal subtraction $(\overline{\mathrm{MS}})$ renormalisation scheme where,

$$
\sin ^{2} \theta_{W}(\mu)_{\overline{\mathrm{MS}}}=e^{2}(\mu)_{\overline{\mathrm{MS}}} / g^{2}(\mu)_{\overline{\mathrm{MS}}},
$$

with $\mu$ representing the sliding energy scale. Theoretically motivated, this definition is, however, unphysical and requires global fits to data in order to obtain an experimental value [5].

The running of $\sin ^{2} \theta_{W}$ with respect to the energy, allows for straightforward testing of the SM. By measuring the weak mixing angle at different energies, one directly examines the accuracy of the SM's predictions. In Fig. 2.2 we show a number of past, present and future low-energy experiments, whose aim is to check the running of $\sin ^{2} \theta_{W} \cdot{ }^{1}$ (Note that in this case $\sin ^{2} \theta_{W}$ is shown as a function of the momentum transfer $Q$ as opposed to $\mu$.)

\subsection{Beyond the SM}

Perhaps one of the most famous examples of agreement between the SM and experiment is the anomalous magnetic moment of the electron,

$$
\begin{aligned}
a_{e}(\mathrm{Exp}) & =11596521807.6 \pm 2.7 \times 10^{-13} \\
a_{e}(\mathrm{SM}) & =11596521817.8 \pm 7.7 \times 10^{-13}
\end{aligned}
$$

where the $a_{e}(\mathrm{SM})[35]$ is the theory prediction. We, however, are primarily interested in those observables which relate to PV in the SM. In Table. 2.2 we show the current state-of-the-art for a number of these measurements, where $C_{1 i}$ and $C_{2 i}$ are defined in terms of the effective four-point interactions,

$$
\mathcal{L}_{\mathrm{NC}}^{e q}=-\frac{G_{F}}{\sqrt{2}} \sum_{i=u, d}\left[C_{1 i} \bar{e} \gamma_{\mu} \gamma^{5} e \bar{q}_{i} \gamma^{\mu} q_{i}+C_{2 i} \bar{e} \gamma_{\mu} e \bar{q}_{i} \gamma^{\mu} \gamma^{5} q_{i}\right]
$$

At tree level,

$$
C_{1 i}=2 g_{A}^{e} g_{V}^{i}, \quad C_{2 i}=2 g_{V}^{e} g_{A}^{i},
$$

\footnotetext{
${ }^{1}$ The final value for $\sin ^{2} \theta_{W}$ extracted from the NuTeV experiment [29] is the subject of considerable debate $[30,31,32,33]$. Despite being shown to lie significantly above the SM prediction in Fig. 2.2, additional corrections such as sea-quark effects result in agreement with theory [28].
} 


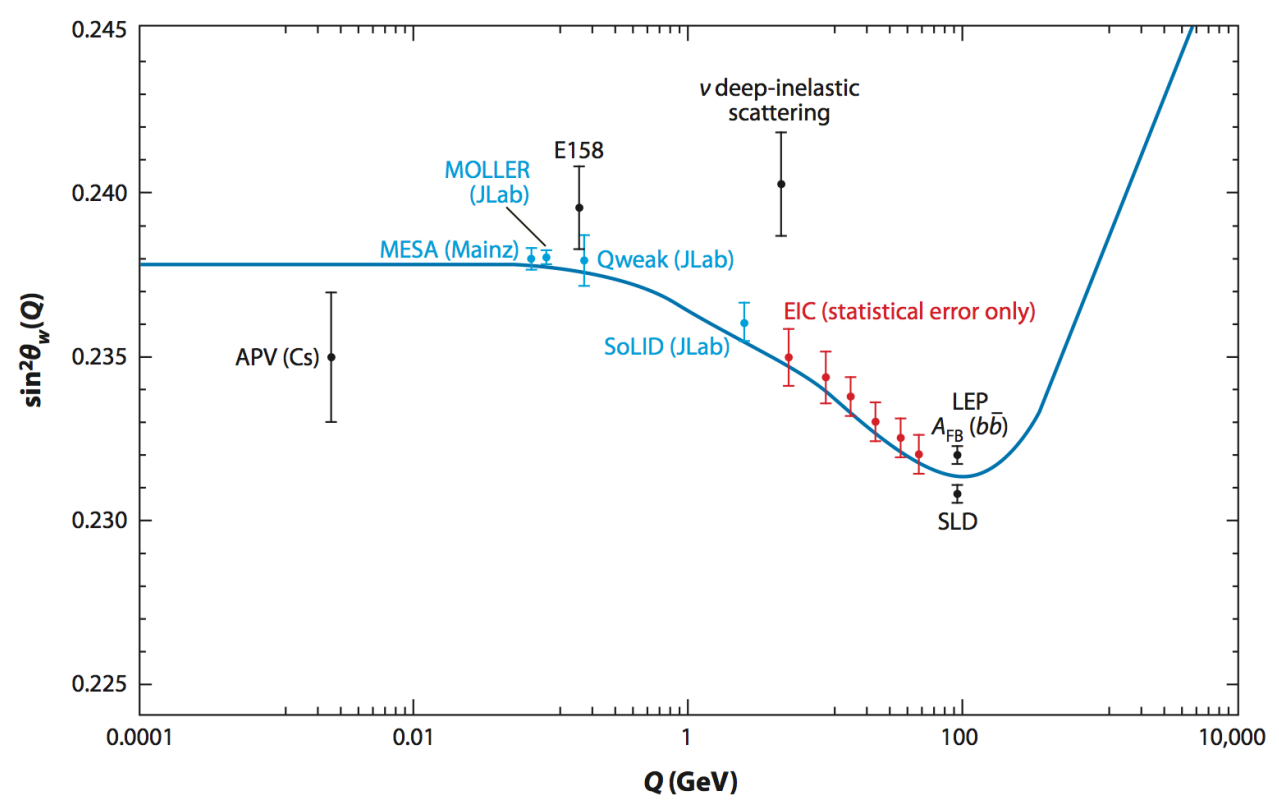

Figure 2.2: The running of $\sin ^{2} \theta_{W}$ and the current experimental status [28]. Black data points represent published results, while the blue and red points are projections for future experiments. The uncertainty on the theory prediction is of order the thickness of the curve, where at low energies this corresponds to $\pm 7 \times 10^{-5}[3,34]$.

where $g_{A}^{e}=-1 / 2$ and $g_{V}^{e}=-\left(1-4 \sin ^{2} \theta_{W}\right) / 2$. The quark couplings,

$$
\begin{aligned}
g_{V}^{u} & =\frac{1}{2}-\frac{4}{3} \sin ^{2} \theta_{W}, & g_{A}^{u} & =\frac{1}{2}, \\
g_{V}^{d} & =-\frac{1}{2}+\frac{2}{3} \sin ^{2} \theta_{W}, & g_{A}^{d} & =-\frac{1}{2},
\end{aligned}
$$

are the same as those in Eq. (2.7).

In spite of the SM's many achievements it seen as incomplete. A number of subatomic and astrophysical observables have lead to the notion that there is a deeper, more fundamental theory, of which the SM is but a low-energy effective theory. Neutrino masses, the rotational speed of galaxies, and the inexplicably low mass of the Higgs boson are all problematic in the SM, and give strength to this understanding. In the following section we expand on some of these difficulties.

\section{Motivating new physics}

Although the list below is by no means complete, it contains many of the most important motivating factors of searches for NP. For a more comprehensive set, see for example, Refs. [5, 36, 37]. 
Table 2.2: Standard Model parity-violating parameters compared with their experimental values [3]. $Q_{W}^{e}$ is the weak charge of the electron.

\begin{tabular}{ccc}
\hline \hline Observable & Experimental value & SM prediction \\
\hline$C_{1 u}+C_{1 d}$ & $0.1537 \pm 0.0011$ & $0.1530(1)$ \\
$C_{1 u}-C_{1 d}$ & $-0.516 \pm 0.014$ & $-0.5300(3)$ \\
$C_{2 u}+C_{2 d}$ & $-0.21 \pm 0.57$ & -0.0089 \\
$C_{2 u}-C_{2 d}$ & $-0.077 \pm 0.044$ & $-0.0627(5)$ \\
$Q_{W}^{e}$ & $-0.0403 \pm 0.0053$ & $-0.0474(5)$ \\
\hline \hline
\end{tabular}

\section{Gravity}

Unlike the strong, weak and electromagnetic forces that can all be described at the quantum level, gravity is currently only successfully realised classically. Attempts to quantise general relativity run into the problem that the resulting theory is non-renormalisable. Furthermore, while it may be possible to combine general relativity to the SM by hand, gravity does not unify with the other three interactions at a fundamental level. It is expected that a more complete theory would include the correct quantum description of gravity as well as unifying it with the SM forces.

\section{Hierarchy problem}

The mass-generating Higgs mechanism results in a neutral scalar boson of spin 0 . Experimental results from the LHC give the mass of the Higgs boson to be $\sim 125 \mathrm{GeV}[1,2]$. Theoretically, however, such a light mass can only be obtained by an artificially large amount of fine tuning.

We may write the Higgs mass as,

$$
m_{H}^{2}=m_{H, \text { bare }}^{2}+\mathcal{O}\left(\lambda, g^{2}, h^{2}\right) \Lambda^{2},
$$

where $\lambda$ is Higgs self-coupling and $g$ and $h$ are the couplings to the gauge bosons and fermions. The bare Higgs mass receives corrections from higher order loop diagrams some of which are shown in Fig. 2.3. These diagrams are proportional to $\Lambda$ - the scale at which the ultraviolet divergences are cut off-and are quadratically divergent. Should there be no physics between the electroweak scale $\left(\Lambda \sim 10^{3} \mathrm{GeV}\right)$ and the Planck scale $\left(\Lambda \sim 10^{19} \mathrm{GeV}\right)$, the cancellation required to get a Higgs mass of $125 \mathrm{GeV}$ must occur to 30 decimal places [5]. Such fine tuning is seen as highly unnatural and any BSM physics which might provide a solution to this problem is considered to be worth examining.

\section{Neutrino masses}

At the time when the SM was originally formulated there was no empirical evidence for massive neutrinos. They were therefore incorporated in the SM as 


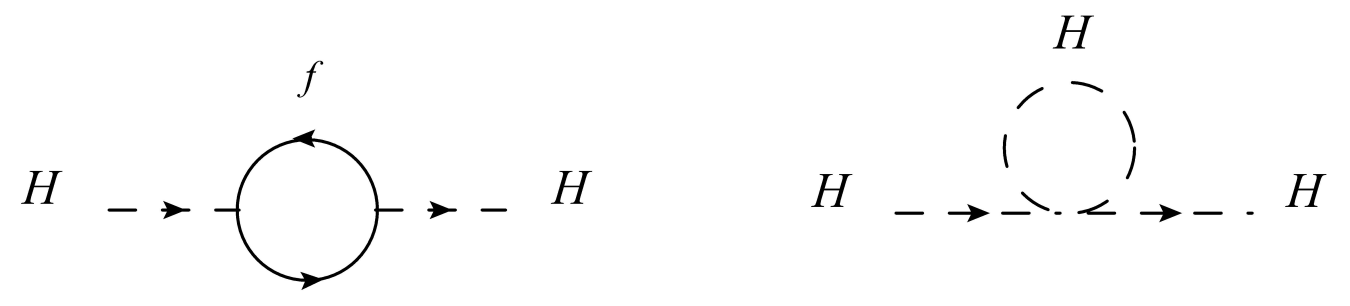

Figure 2.3: Examples of one loop corrections to the physical Higgs mass.

massless particles. Since then however, experimental data $[38,39,40]$ indicates that neutrinos oscillate, i.e. they change flavour as they propagate. This means that in fact the neutrinos must have some mass, albeit very small. Although masses for neutrinos may be incorporated into the SM by either adding extra right-handed states, or by the 'seesaw mechanism' [41, 42, 43], there is still no experimental evidence to decide between the two scenarios. Since these additions are not a part of the original SM, NP is still needed to offer a more satisfactory solution.

Another question which the SM fails to answer is whether neutrinos are Dirac or Majorana particles.

\section{Gauge coupling unification}

In the SM, the effective gauge couplings - corresponding to the strength of the forces - may be written as functions of the energy scale. If the running of couplings are plotted together as shown in Fig. 2.4, it is clear that they do not meet at a single point. The failure of the coupling constants to unify is seen as a drawback of the SM and NP is required to obtain such unification.

\section{Dark matter}

Gravitational lensing, star velocities in spiral galaxies and large-scale cosmological structure all suggest that the universe contains additional non-nuclear matter. Invisible electromagnetically, thus 'dark', it is thought to make up $22 \%$ of the total energy content of the universe [5]. Although its presence is currently inferred only from gravitational interactions, it is also possible that dark matter (DM) interacts weakly.

While there have been several SM based explanations for dark matter, such as neutrinos, or 'MACHOs', (Massive Compact Halo Objects) most of these candidates fail to explain the full range of current observations. In the case of neutrinos, their masses are too light to give the correct relic density and their velocities too high to allow for formation of the observed large scale structures. MACHOs on the other hand, lack a credible mechanism for creating the amount of objects needed to account for the masses of the galaxies.

The failure of the SM to offer a suitable solution for DM, has left physicists little option but to look outside the SM. A number of candidates e.g. 


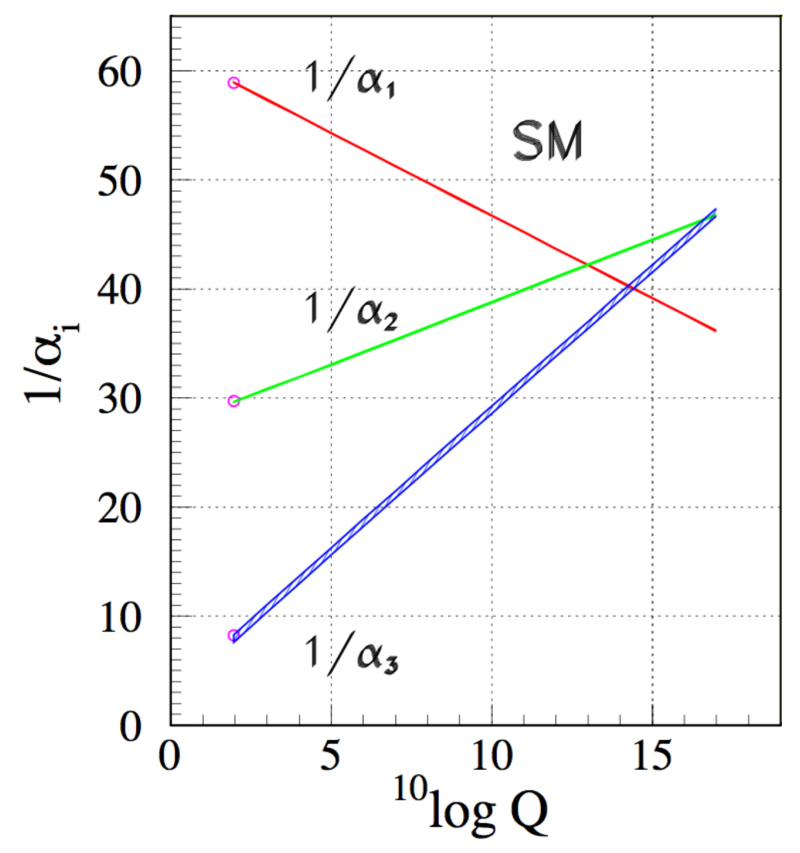

Figure 2.4: Non-unification of the gauge couplings in the SM [44]. The couplings are defined as $\alpha_{1} \equiv 5 / 3\left[g^{2} /(4 \pi)\right], \alpha_{2} \equiv g^{2} /(4 \pi)$ and $\alpha_{3} \equiv g_{s}^{2} /(4 \pi)$.

axions, gravitinos, even non-Newtonian gravity have all at various points been considered as possible explanations. The leading candidate, however, remains weakly interacting massive particles (WIMPs) and to be given serious attention most BSM theories require such a particle.

\section{Candidates for BSM physics}

Given the range of issues with the SM, it is perhaps not surprising that there is no shortage of contenders for alternative physics models. Although there is yet to be any experimental verification of any of these theories - either via direct or indirect detection - there remains a large body of work devoted to studying their implications. This next section looks at some of the more prominent BSM theories.

\section{Supersymmetry}

One of the best theoretically motivated extensions of the SM is supersymmetry (SUSY). Originally introduced by Wess and Zumino [45], SUSY works by extending the Poincaré algebra [46] to include spinor generators. This successfully negotiates the Coleman-Mandula No-go theorem [47], which states that the most general symmetry group of the S-Matrix is the cross product of the spacetime transformations and an internal symmetry (such as a gauge theory).

The spinor generators $Q_{\alpha}$ act on fermions (bosons) transforming them 
to bosons (fermions),

$$
\begin{aligned}
Q_{\alpha}|\mathrm{f}\rangle & =|\mathrm{b}\rangle, \\
Q_{\alpha}|\mathrm{b}\rangle & =|\mathrm{f}\rangle .
\end{aligned}
$$

This means that each particle acquires an additional superpartner - the fermions a bosonic partner, and the bosons a fermionic partner. The Higgs sector is, moreover, extended to two doublets.

One of the most attractive properties of SUSY is that it provides a natural DM particle. WIMPs are considered prime candidates for dark matter because they are both heavy enough $\left(\sim 10^{2}-10^{3} \mathrm{GeV}[5]\right)$, to allow for the observed rotation speeds of the stars and only weakly interacting, accounting for inability to detect it through the electromagnetic spectrum. With the addition of so-called R-parity - necessary for proton stability - the lightest supersymmetric particle (LSP) becomes stable making it an ideal DM possibility.

SUSY theories may also help solve the hierarchy problem. The Higgs self-energy loops containing additional supersymmetric particles cancel out those that appear in the SM (cf. Fig. 2.3), removing the need for artificial fine tuning. In combination with grand unified theories, SUSY also allows for the unification of the coupling constants. Another attractive quality of SUSY is that it opens up the possibility of a quantum description of gravity.

Because SUSY provides solutions to many of the SM's problems, it is one of the most extensively studied BSM theories. Experimentally, there are a considerable number of searches under way at the LHC. In detectors such as ATLAS and CMS, the LSP may be inferred from missing energy signatures. Other astrophysical experiments hope to see signs of SUSY particles indirectly by measuring self-annihilation products such as gamma rays.

\section{Extra dimensions}

The LHC currently looks for signs of extra spatial dimensions also. The simplest extension to the standard four-dimensional spacetime involves a fifth, circular spatial dimension $[48,49]$. Given that this additional dimension has not yet been observed, its radius should be small. This extra dimension then results in a tower of massive states called a Kaluza-Klein tower. These momentum states may be searched for in modern detectors.

Theoretical motivation for extra dimensions comes from both string theory and the ability to incorporate gravity with the three other forces. As of today, there is yet to be any experimental evidence for extra dimensions.

\section{Technicolour}

Finally, we mention technicolour. In these types of theories electroweak symmetry is broken by an additional non-abelian gauge interaction removing the need for a scalar (Higgs) field and the associated hierarchy problem. This is attractive since it removes the fine tuning that might otherwise appear. 


\section{Experimental searches}

The LHC is just one of a multitude of experiments currently under way, whose goal is to discover new physics. These searches are often divided into three 'frontiers' - the Energy, Intensity, and Cosmic frontiers. Although they incorporate more than just BSM experiments these divisions are still useful classification tools. In this section we give a broad overview of these NP searches, for a more complete review see Refs. [5, 28].

\section{Energy frontier}

This category is dominated by the Large Hadron Collider. With four detectors (ATLAS, CMS, ALICE and LHCb) and an anticipated final centre-of-mass energy of $14 \mathrm{TeV}$, the LHC is the largest and most powerful particle accelerator yet created. Its range of NP searches includes all those previously discussed i.e. supersymmetry [50], extra dimensions [51] and technicolour [52], as well as others like microscopic black holes [53]. Although they have yet to observe any of these phenomena, these experiments have placed new constraints on the energy scales of BSM physics. With the upgrade of the LHC almost complete, a new range of experiments will begin which will continue to increase these limits. Other planned experiments at the energy frontier include the International Linear Collider and the Future Circular Collider.

Unfortunately, the nature of the LHC experiments means that it would be difficult to discover the precise properties of any NP observed. To do this requires experiments at the intensity frontier.

\section{Intensity frontier}

This technique provides an alternative approach to the 'brute-force' method discussed above. In these types of experiments, beams of high intensity are focused on a target resulting in measurements which are repeated many times over. By building up the statistics and ensuring that the systematic errors are under control, these experiments make precision measurements of SM observables with hope of detecting 'smoking-gun' signals of new physics.

The previously mentioned $Q_{\text {weak }}$ experiment [54], MOLLER [55] and the E08-011 experiment [56], all fall under this category. Further examples include the proposed $11 \mathrm{GeV}$ PVDIS [57] experiment, the measurement of the proton radius [58] and those involving the neutrino mixing parameters such as in Ref. [59].

Another important field of research which falls within this grouping is the Cabibbo-Kobayashi-Maskawa (CKM) quark-mixing matrix [60, 61]. One of the main aims of flavour physics is to precisely determine the CKM matrix elements. Experiments such as those at Belle [62], BaBar [63] and LHCb [64] all play significant roles in this area. 


\section{Cosmic frontier}

In this category, observations are done on the cosmos. Optical, radio and even 'neutrino' telescopes such as ICECUBE are used to provide a better picture of the Universe. Techniques such as gravitational lensing have delivered key evidence [65] for dark matter and further observations may well answer remaining questions on the nature of DM. Additionally, studies of cosmic rays $[66,67]$ provide windows into particle collisions at energies many orders of magnitude greater than those currently available at modern accelerators. 


\section{Precision tests of the SM}

While in some sense any experiment performed is a test of the Standard Model, precision tests refers specifically to those whose uncertainties are significantly smaller than previous measurements in the field. It is of course, also necessary that the theory prediction be known very precisely. Several examples have already been mentioned in the previous two chapters and although the range of experiments which fall under this category is too large to fully account for in this chapter, here we briefly point out some of the key findings.

Following the establishment of the basic structure of the SM in the 1980s, the next decade saw precision measurements involving $Z$ physics at the Large Electron-Position Collider (LEP) and Stanford Linear Collider (SLC). These experiments confirmed the validity of the SM to the one-loop level [68]. The 2000s saw precision measurements of the top quark mass [69], the $W$-boson mass [70], as well as the muon anomalous magnetic moment [71] and the Fermi constant [72]. More recently, the mass generating mechanism in the SM has been confirmed with the discovery of the Higgs boson $[1,2]$.

In the next two sections we present additional details on the precision experiments relevant to calculations performed later on in this thesis. For further information on electroweak tests, the reviews $[28,73,74,75]$ are particularly helpful.

\subsection{Atomic parity violation}

Neutral current PV experiments may be separated into a number of categories. Atomic parity violation (APV), parity-violating electron scattering (PVES) and neutrino scattering measurements are three of the main kinds of experiments used in this field. For the purposes of this thesis we concentrate on the former two. In APV, electron-nucleus interactions mediated by the $Z$-boson result in PV transitions between energy states of the atom. By measuring the amplitude for this transition, one is sensitive to the value of the weak charge,

$$
Q_{W}(Z, N)=Z\left(1-4 \sin ^{2} \theta_{W}\right)-N,
$$

where $Z$ is the number of protons and $N$, neutrons. One of the benefits of APV measurements is that they avoid the difficulty scattering experiments 
face where the need for high statistics is hindered by the target over heating. While the required atomic theory calculations are still technically challenging, experimentally, this makes it more straightforward to determine $\sin ^{2} \theta_{W}$ at lower energies in APV.

The earliest efforts to observe PV in neutral currents attempted to measure APV in bismuth-209 [18, 20] and although these experiments failed to give a definitive answer, since then, PV has been observed in ${ }^{209} \mathrm{Bi}$ and other nuclei at the expected level $[19,76,77,78]$. The most precise measurement of APV to date, however, comes from the highly forbidden $6 S \rightarrow 7 S$ transition in cesium-133 [79, 80, 81], where they determined,

$$
Q_{W}=-72.06(28)_{\exp }(34)_{\text {theor }}
$$

a value more than two standard deviations away from the SM prediction at the time. Since then, the extracted value of the weak mixing angle has shifted as new corrections $[2,82,83]$ have been taken into account and it is currently considered to be

$$
\sin ^{2} \theta_{W}=0.2212(19),
$$

which sits $1.5 \sigma$ away from the SM value (shown in Fig. 2.2).

\subsection{Parity-violating electron scattering}

Parity-violating scattering experiments, involve a beam of polarised leptons (usually electrons) on some stationary, nuclear target. The cross sections for the left- and right-handed polarised leptons are measured and the differencenormalised by the total-gives an asymmetry which is sensitive to $\sin ^{2} \theta_{W}$. The advantage of these experiments is that the ambiguities surrounding atomic theory corrections in APV measurements are no longer present. Following the original work of Prescott et al. [21, 22], there have been many further PVES experiments [54, 84, 85, 86, 87, 88]. Of these, the Møller scattering E158 experiment [84] at the Stanford Linear Accelerator Center (SLAC), provides the best measurement of $\sin ^{2} \theta_{W}$ away from the $Z$-pole. Many of the other experimental results will be useful, in conjunction with $Q_{\text {weak }}$, in further constraining the proton's weak charge.

\section{$Q_{\text {weak }}$}

Precision measurements of $Q_{W}^{p}$ are particularly well suited to searches for NP since ad hoc cancellations suppress the weak charge's value in the SM [89]. Furthermore, previous PVES experiments $[54,85,86,87,88]$ may be used to reduce remaining hadronic uncertainties which might otherwise be difficult to estimate theoretically.

Of principal interest to this work is the $Q_{\text {weak }}$ experiment [54] at JLab. Like those mentioned above, it is a PVES experiment and has recently completed the data taking stage. Longitudinally polarised electrons with energy 
$E=1.165 \mathrm{GeV}$ are elastically scattered off a fixed proton (liquid hydrogen) target. The resulting four-momentum transfer is $Q^{2}=0.025 \mathrm{GeV}^{2}$.

The difference in the helicity-dependent cross sections is measured by the asymmetry,

$$
A_{\mathrm{PV}}=\frac{\sigma^{+}-\sigma^{-}}{\sigma^{+}+\sigma^{-}}
$$

where $\sigma_{\lambda}$ is the cross section for a right-hand $(\lambda=+1)$ or left-hand $(\lambda=-1)$ electron. With its aim of measuring proton's weak charge to $4 \%, Q_{\text {weak }}$ would determine $\sin ^{2} \theta_{W}$ to $\sim 0.3 \%$, a precision approaching those of the $Z$-pole measurements $(0.1 \%)$. Should $Q_{\text {weak }}$ reach this goal, it would supplant the E158 experiment as the most precise value of the weak mixing angle at $Q^{2} \ll$ $m_{Z}^{2}$.

$Q_{\text {weak }}$ recently released a value of $Q_{W}^{p}$ following an analysis of $4 \%$ of the data. Using results from presented in this thesis [90], they found $Q_{W}^{p}=$ $0.0710 \pm 0.0007$ [91]. The analysis of the full data set is expected to be completed by late 2014. Should this value agree with SM prediction, it will constrain new physics at the 1-5 TeV scale [92]. In Sections 3.3 and 3.4 we will look more closely at how higher order radiative corrections, especially the $\square_{\gamma Z}$ correction, effects the SM prediction of $Q_{W}^{p}$. We will find that when trying to place constraints on NP, the magnitude of the uncertainty of the $\square_{\gamma Z}$ correction is particularly important.

\section{PVDIS at $6 \mathrm{GeV}$}

This is an $e^{-} d$ scattering experiment similar to the Prescott's earlier set up $[21,22]$. In fact, it is the first experiment since then to measure the inelastic asymmetry in the DIS region. Using JLab's $6 \mathrm{GeV}$ beam it measured $A_{\mathrm{PV}}$ over a range of kinematics with the primary aim of determining the poorly known axial-vector quark couplings which were defined in Section 2.2. Later on, we will use the results from this experiment $[56,93]$ to test the consistency of the AJM model.

\section{MOLLER}

Of a similar nature to the E158 experiment, MOLLER [55] aims to measure the electron's weak charge to $2.3 \%$ - equivalent to measuring $\sin ^{2} \theta_{W}$ to $\approx 0.1$ $\%$ - placing it on par with the two measurements done by LEP at the $Z$-pole. In order to reach this precision, it will need to be able to detect scattered electrons at very forward angles, as well as ensuring high enough statistics.

As in the SLAC measurement, longitudinally polarised electrons will scatter off the atomic electrons in hydrogen. One additional benefit is that it will include, in its backgrounds, a $4 \%$ measurement of the proton's weak charge [94]. MOLLER will also measure the proton's inelastic asymmetry at low momentum transfer [95], which will provide additional constraints on the proton's interference structure functions. 


\section{Further measurements}

In addition to those above, there are several other planned experiments which aim to measure the weak mixing angle to even higher levels of precision. SoLID [57] is a proposed update of the $6 \mathrm{GeV}$ PVDIS experiment which will run after the completion of the $12 \mathrm{GeV}$ energy upgrade to JLab's Continuous Electron Accelerator Facility (CEBAF). Improving on the earlier experiment, it will included a larger number of $A_{\mathrm{PV}}$ values as well as increasing the precision to $0.5-1 \%$ with the goal of more precisely determining the neutral current vector and axial-vector quark couplings [28]. As in previous cases, this will also provide an independent low-energy measurement of $\sin ^{2} \theta_{W}$.

Another proposed experiment is the P2 at the Mainz Energy-recovering Superconducting Accelerator (MESA) facility. P2 hopes to measure the proton's weak charge using a beam energy of $\sim 0.2 \mathrm{GeV}$ which would reduce theoretical controversies regarding hadronic box corrections such as $\square_{\gamma Z}$. Perhaps even more optimistically, as a part of the proposed Electron-Ion Collider (EIC) plans, $\sin ^{2} \theta_{W}$ could be measured at several values of $Q$ with the possible (red) data points shown in Fig. 2.2. This would clearly be an extremely good test of the slope of the curve of $\sin ^{2} \theta_{W}$.

$* \quad * \quad *$

Having presented an overview of PV experiments in the above section, the rest of the chapter focuses solely on the theoretical aspects of the $Q_{\text {weak }}$ experiment. Since we are specifically interested in the $\square_{\gamma Z}$ radiative correction to the proton's weak charge, the next two sections are dedicated to introducing this topic. In Section 3.3 general radiative corrections to $Q_{W}^{p}$ are discussed, while in Section 3.4 we look exclusively at the $\square_{\gamma Z}$ contribution. It is important to remember, however, that a number of the experiments mentioned above will play an important role in any future determination of $Q_{W}^{p}$.

\subsection{Radiative corrections to $Q_{W}^{p}$}

Many of the radiative corrections discussed in this section were originally calculated in the context of APV experiments. The work of Marciano and Sirlin $[96,97]$ provides the basis for the more modern calculations [98] which look specifically at PV in electron scattering. Indeed, part of the recent confusion surrounding the $\square_{\gamma Z}$ diagram stemmed from the fact that APV and PVES experiments are characterised by different energies. As the individual components of the $\square_{\gamma Z}$ diagram each exhibit distinct energy dependence, it is imperative to ensure that this is correctly determined.

Beginning again with the asymmetry in Eq. (3.4), $Q_{\text {weak }}$ measures the difference between the left- and right-handed electron cross sections. Given that these cross sections are proportional to the square of the amplitude, $|\mathcal{M}|^{2}$, 
where,

$$
\begin{aligned}
|\mathcal{M}|^{2} & =\left|\mathcal{M}_{\gamma}+\mathcal{M}_{Z}\right|^{2} \\
& =\left|\mathcal{M}_{\gamma}\right|^{2}+2 \mathcal{R} e\left(\mathcal{M}_{\gamma}^{*} \mathcal{M}_{Z}\right)+\left|\mathcal{M}_{Z}\right|^{2}
\end{aligned}
$$

and $\mathcal{M}_{\gamma}$ is the electromagnetic Born amplitude, while $\mathcal{M}_{Z}$ is the Born $Z$ exchange amplitude, it is clear that the PV part comes predominantly from the $2 \mathcal{R} e\left(\mathcal{M}_{\gamma}^{*} \mathcal{M}_{Z}\right)$ term. This is because the $\left|\mathcal{M}_{\gamma}\right|^{2}$ will cancel in the numerator of Eq. (3.4) while $\left|\mathcal{M}_{Z}\right|^{2}$ is negligible at the kinematics of $Q_{\text {weak }}$. The denominator on the other hand will be dominated by the $\left|\mathcal{M}_{\gamma}\right|^{2}$ piece.

In the case where the momentum transfer squared $t$, is small, $A_{\mathrm{PV}}$ is related to the proton's weak charge by [73]

$$
A_{\mathrm{PV}}=\frac{G_{F}}{4 \pi \alpha \sqrt{2}} t Q_{W}^{p},
$$

where $\alpha$ is the fine structure constant and $G_{F}$ is the Fermi constant. Further, the weak charge and the Weinberg angle are related at tree level by the expression,

$$
Q_{W}^{p}=1-4 \sin ^{2} \theta_{W} .
$$

At the level of precision required by $Q_{\text {weak }}$, however, radiative corrections must also be included, giving [98],

$$
Q_{W}^{p}=\left(1+\Delta \rho+\Delta_{e}\right)\left(1-4 \sin ^{2} \theta_{W}(0)+\Delta_{e}^{\prime}\right)+\square_{W W}+\square_{Z Z}+\square_{\gamma Z}(0),
$$

where $\sin ^{2} \theta_{W}(0)$ is the weak mixing angle at zero momentum and $\square_{\gamma Z}(0)$ is the $\gamma Z$ box diagram at $E=0$. Almost all the additional corrective terms have been calculated to the levels of precision necessary for $Q_{\text {weak }}$. The only term currently subject to debate is the $\square_{\gamma Z}$ box diagram.

Looking at the individual corrections in Eq. (3.8), the $\Delta \rho$ term gives the correction to the relative normalisation of the neutral and charged current amplitudes. Both higher order QCD corrections and the electroweak equivalents have been included in its evaluation. Marciano and Sirlin give $\Delta_{e}=-\alpha / 2 \pi$, while the electron's anapole moment $\Delta_{e}^{\prime}$ is,

$$
\Delta_{e}^{\prime}=-\frac{\alpha}{3 \pi}\left(1-4 s_{W}^{2}\right)\left[\ln \left(\frac{M_{Z}^{2}}{m_{e}^{2}}\right)+\frac{1}{6}\right]
$$

where $s_{W}^{2}=\sin ^{2} \theta_{W}[96,97]$. These are relatively small corrections to the axial-vector Zee and ree couplings [98].

The electroweak box diagrams $\square_{W W}$ and $\square_{Z Z}$ have been computed by the authors of Refs. [96, 97, 99] who found,

$$
\square_{W W}=\frac{7 \alpha}{4 \pi s_{W}^{2}},
$$



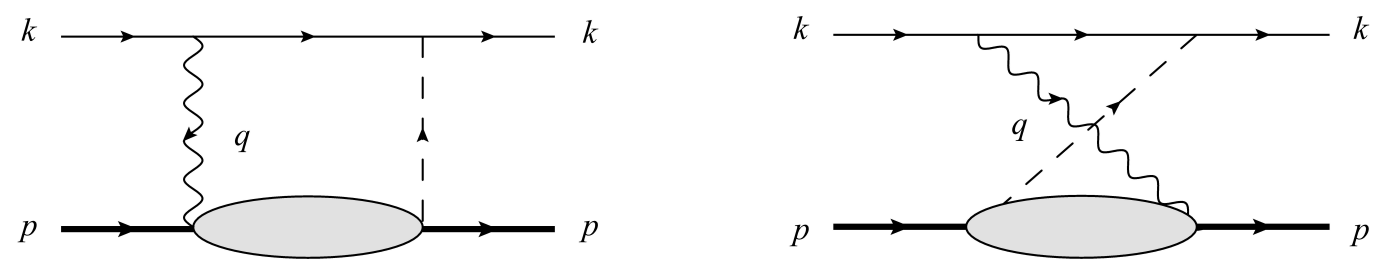

Figure 3.1: Interference $\gamma Z$ box (left) and crossed box (right) diagrams. The wavy and dashed lines represent the exchanged $\gamma$ and $Z$ bosons, with the hadron, electron and virtual photon momenta labelled by $p, k$, and $q$, respectively.

and

$$
\square_{Z Z}=\frac{\alpha}{4 \pi s_{W}^{2} c_{W}^{2}}\left(\frac{9}{4}-5 s_{W}^{2}\right)\left(1-4 s_{W}^{2}+8 s_{W}^{2}\right),
$$

where $c_{W}^{2}=\cos ^{2} \theta_{W}$ and here, both $\alpha$ and $\theta_{W}$ take their $Z$-pole values and have been renormalised in the modified minimal subtraction scheme. Given that the $\square_{W W}$ diagram contributes $\sim 26 \%$ to $Q_{W}^{p}$, it is particularly important that this contribution be well understood. The $\square_{Z Z}$ diagram on the other hand accounts for a much smaller $\sim 3 \%$.

In both the $\square_{W W}$ and $\square_{Z Z}$ diagrams, the majority of the contribution comes from the DIS region [98] where perturbation theory may be used. For lower kinematics where this is no longer the case, the diagrams carry suppression factors involving $\left(p / M_{W, Z}\right)^{2}$ (where $p$ is the incoming momentum) and may thus be neglected [98]. This combination of factors results in values for $\square_{W W}$ and $\square_{Z Z}$ which are well within $Q_{\text {weak }}$ uncertainty limits.

\subsection{The $\square_{\gamma Z}$ correction}

Until more recently, the interference $\gamma Z$ contribution shown in Fig. 3.1 was also thought to be known within the required precision of the $Q_{\text {weak }}$ experiment. In terms of the electroweak amplitudes, it is defined as [100]

$$
\square_{\gamma Z}(0)=Q_{W}^{p} \frac{\Re e\left(\mathcal{M}_{\gamma}^{*} \mathcal{M}_{\gamma Z}^{(\mathrm{PV})}\right)}{\Re e\left(\mathcal{M}_{\gamma}^{*} \mathcal{M}_{Z}^{(\mathrm{PV})}\right)},
$$

where $\mathcal{M}_{Z}^{(\mathrm{PV})}$ and $\mathcal{M}_{\gamma Z}^{(\mathrm{PV})}$ are the parity-violating parts of the $Z$ and $\gamma Z$ interference amplitudes.

The $\square_{\gamma Z}$ diagram may be decomposed into two separate pieces,

$$
\square_{\gamma Z}(E)=\square_{\gamma Z}^{A}(E)+\square_{\gamma Z}^{V}(E),
$$

with $\square_{\gamma Z}^{A}$ coming from the vector electron, axial-vector hadron coupling to the $Z$ boson and $\square_{\gamma Z}^{V}$ from the axial-vector electron, vector hadron coupling to the $Z$. 
At typical APV energies $\mathcal{O}(\mathrm{MeV})$, the hadronic axial-vector correction dominates, since $\square_{\gamma Z}^{V} \rightarrow 0$ as the energy decreases and is therefore negligible in such experiments. For $Q_{\text {weak }}$ on the other hand, $E=1.165 \mathrm{GeV}$ which is approximately three orders of magnitude larger. At these energies $\square_{\gamma Z}^{V}$ is no longer negligible, thus both terms need to be taken into account. Although the majority of our analysis will focus on the vector hadron correction, in the next few paragraphs we give a brief summary of the current state of the $\square_{\gamma Z}^{A}$ contribution.

Marciano and Sirlin's (MS) original calculations separated $\square_{\gamma Z}^{A}$ into a high and low energy part [96, 97, 98],

$$
\square_{\gamma Z}^{A}=\frac{5 \alpha}{2 \pi}\left(1-4 s_{W}^{2}\right)\left[\ln \frac{M_{Z}^{2}}{\lambda^{2}}+C_{\gamma Z}(\lambda)\right] .
$$

The parameter $\lambda \sim 1 \mathrm{GeV}$ represents the scale of separation, $C_{\gamma Z}$ gives the low-energy contribution and the log factor, the high-energy contribution. MS estimated the low-energy contribution using the Born term for the $\gamma Z$ interference diagram that is, instead of including all the states represented in Fig. 3.1, they only calculated the contribution from the diagram with the proton as the intermediate state. For the short distance contribution, the quark parton model was used.

Blunden et al. (BMT) [101, 102] have recently updated this calculation using the dispersion formalism. While (slightly) improving the precision, they found that the central values remained in good agreement with the Refs. [96, 97]:

$$
\begin{aligned}
& \square_{\gamma Z}^{A}=0.0052(5) ; \quad \mathrm{MS} \\
& \square_{\gamma Z}^{A}=0.0044(4) ; \quad \text { BMT }
\end{aligned}
$$

where the above values are being compared at $E=0$.

There is little argument over the axial-vector hadron contribution at $Q_{\text {weak }}$ energies - mostly because the majority of the contribution comes from the DIS region where perturbative QCD can still be used. This is in contrast to the $\square_{\gamma Z}^{V}$ term, where the long distance physics plays a much more important role. Finally, the $\square_{\gamma Z}^{A}$ correction has now been calculated $[103,104]$ up to the MOLLER experiment's energy of $11 \mathrm{GeV}$ and its uncertainty remains well within the error budget of both $Q_{\text {weak }}$ and MOLLER.

Aside from including the dispersion expressions for the $\square_{\gamma Z}^{A}$ for completeness' sake, the rest of this analysis will now focus on the $\square_{\gamma Z}^{V}$ contribution whose uncertainty has been subject to much wider debate. Note that since the elastic contribution has already been evaluated previously Refs. [96, 97, 105, 106] we will not spend any more time on it here. Additionally since it is suppressed by an extra $Q_{W}^{p}$ factor, the elastic part is significantly smaller than the inelastic $\square_{\gamma Z}^{V}$ values determined here.

Gorchtein and Horowitz [107] first applied the dispersion framework to the $\square_{\gamma Z}$ term and showed that because of its strong energy dependence, its 


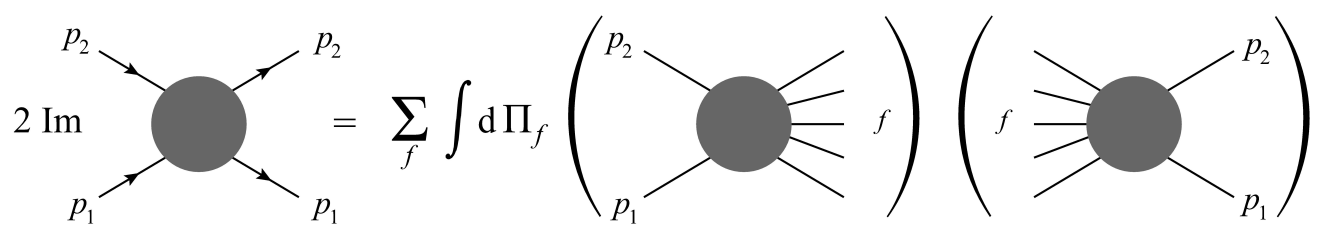

Figure 3.2: Optical theorem which states that the imaginary part of the forward amplitude is equal to the sum of all possible intermediate states [111].

contribution at $Q_{\text {weak }}$ energies was much larger than previously thought [98]. Moreover, they found that the uncertainty on this term was much larger than Marciano and Sirlin's earlier estimate. Since this would affect the precision aims of $Q_{\text {weak }}$ it resulted in considerable concern.

A follow-up calculation by Sibirtsev et al. [108] agreed within errors with the central value, but showed that Ref. [107] suffered from a number of mistakes including a missing factor of two. The authors of Ref. [108] also argued that the errors had been overestimated and that it was more reasonable to assume smaller uncertainties. Rislow and Carlson (in Ref. [109]) again confirmed the magnitude of the overall value of Gorchtein and Horowitz, but agreed with the smaller values of uncertainty of Ref. [108].

Since then, Gorchtein et al. [110] revisited their earlier calculation, including a more robust discussion of the role of model dependence in $\square_{\gamma Z}$. They also corrected the errors in their previous work. However, in regards to the uncertainty they maintained that this was still more than twice that of those found in Refs. [108] and [109]. As a result of this wide range of uncertainty values, a detailed analysis of the evaluations was needed in order to move past the confusion. Before moving on to discuss the different models that have been used in the literature, the rest of this chapter will be used to introduce the dispersion formalism.

At forward angles, the real part of the $\square_{\gamma Z}^{V, A}$ corrections can be calculated from their imaginary part using the dispersion relations,

$$
\begin{aligned}
\Re e \square_{\gamma Z}^{V}(E) & =\frac{2 E}{\pi} \mathcal{P} \int_{0}^{\infty} d E^{\prime} \frac{1}{E^{\prime 2}-E^{2}} \Im m \square_{\gamma Z}^{V}\left(E^{\prime}\right), \\
\Re e \square_{\gamma Z}^{A}(E) & =\frac{2}{\pi} \mathcal{P} \int_{0}^{\infty} d E^{\prime} \frac{E^{\prime}}{E^{\prime 2}-E^{2}} \Im m \square_{\gamma Z}^{A}\left(E^{\prime}\right),
\end{aligned}
$$

where $\mathcal{P}$ refers to the Cauchy principal value integral and the crossed terms are included in the above integrals. Note also that from Eq. (3.15) it is clear why this contribution vanishes as $E \rightarrow 0$ and conversely why the axial hadron term remains finite at zero energy thus dominating the APV calculations.

From the optical theorem - illustrated diagrammatically in Fig. 3.2 - the imaginary part of the interference amplitude may be written in terms of the 
leptonic $\left(L_{\mu \nu}\right)$ and hadronic $\left(W_{\mu \nu}\right)$ tensor [100, 107, 108],

$$
2 \Im m \mathcal{M}_{\gamma Z}^{(\mathrm{PV})}=-4 \sqrt{2} \pi M G_{F} \int \frac{d^{3} k^{\prime}}{(2 \pi)^{3} 2 E_{k^{\prime}}}\left(\frac{4 \pi \alpha}{Q^{2}}\right) \frac{1}{1+Q^{2} / M_{Z}^{2}} L_{\mu \nu}^{\gamma Z} W_{\gamma Z}^{\mu \nu},
$$

where $k^{\prime}=k-q$ is the outgoing electron momentum. The $\gamma Z$ lepton tensor is defined to be,

$$
L_{\mu \nu}^{\gamma Z}=\bar{u}(k, \lambda)\left(g_{V}^{e} \gamma_{\mu}-g_{A}^{e} \gamma_{\mu} \gamma_{5}\right) \not k^{\prime} \gamma_{\nu} u(k, \lambda),
$$

and we remind the reader that $g_{V}^{e}=-\left(1-4 \sin ^{2} \theta_{W}\right) / 2$ (at tree level) and $g_{A}^{e}=-1 / 2$ are the vector and axial-vector couplings of the electron to the weak current, while $\lambda$ is the lepton helicity. The nucleon initial state hadronic tensor is

$$
M W_{\gamma Z}^{\mu \nu}=-g^{\mu \nu} F_{1}^{\gamma Z}+\frac{p^{\mu} p^{\nu}}{p \cdot q} F_{2}^{\gamma Z}-i \epsilon^{\mu \nu \lambda \rho} \frac{p_{\lambda} q_{\rho}}{2 p \cdot q} F_{3}^{\gamma Z}
$$

where the $F_{i}^{\gamma Z}$ are the interference structure functions and $\epsilon^{\mu \nu \lambda \rho}$ is the totally antisymmetric tensor. These structure functions are analogues of the better experimentally determined electromagnetic structure functions.

Equations (3.18) and (3.19) can be combined to give the imaginary parts of the hadron vector and axial-vector amplitudes in terms of the $\gamma Z$ structure functions [100, 107, 108],

$$
\begin{aligned}
\Im m \square_{\gamma Z}^{V}(E)=\frac{1}{\left(s-M^{2}\right)^{2}} & \int_{W_{\pi}^{2}}^{s} d W^{2} \int_{0}^{Q_{\max }^{2}} d Q^{2} \frac{\alpha\left(Q^{2}\right)}{1+Q^{2} / M_{Z}^{2}} \\
& \times\left[F_{1}^{\gamma Z}+\frac{s\left(Q_{\max }^{2}-Q^{2}\right)}{Q^{2}\left(W^{2}-M^{2}+Q^{2}\right)} F_{2}^{\gamma Z}\right],
\end{aligned}
$$

and $[100,101,102]$,

$$
\begin{aligned}
\Im m \square_{\gamma Z}^{A}(E)=\frac{1}{\left(s-M^{2}\right)^{2}} & \int_{W_{\pi}^{2}}^{s} d W^{2} \int_{0}^{Q_{\max }^{2}} d Q^{2} \frac{v_{e}\left(Q^{2}\right) \alpha\left(Q^{2}\right)}{1+Q^{2} / M_{Z}^{2}} \\
& \times\left[\frac{2 M E}{W^{2}-M^{2}+Q^{2}}-\frac{1}{2}\right] F_{3}^{\gamma Z},
\end{aligned}
$$

where $v_{e}\left(Q^{2}\right)=1-4 s_{W}^{2}\left(Q^{2}\right)$, the square of the total centre-of-mass energy is $s=M^{2}+2 M E, W_{\pi}^{2}=\left(M+m_{\pi}\right)^{2}$ is the invariant mass at pion-production threshold and $Q_{\max }^{2}=2 M E\left(1-W^{2} / s\right)$. As in Ref. [101] we include the $Q^{2}$ dependence of the fine structure constant $\alpha\left(Q^{2}\right)$.

As it turns out, the most important aspect of the $\square_{\gamma Z}^{V}$ calculation revolves around the $\gamma Z$ structure function inputs. A complete knowledge of these structure functions would allow for a precise determination of the correction leaving little room for debate. This is not the case, however, for although the electromagnetic structure functions have been determined accurately in 
the crucial low- $Q^{2}$, low- $W^{2}$ regions by experiments like E94-110 [112, 113] at JLab, there is no such data for the $\gamma Z$ structure functions. There have been extractions of $F_{2}^{\gamma Z}$ and $x F_{3}^{\gamma Z}$ at much larger values of $Q^{2}$ and $W^{2}$ by the H1 collaboration [114], but this region contributes only a small amount to the total box correction. Given the lack of empirical data, one must instead used models of these structure functions as inputs into the expression for $\square_{\gamma Z}^{V}$. As it is difficult to distinguish between models, the debate surrounding this correction is perhaps not surprising.

In the next chapter we will review the current set of structure function models used to determine the $\square_{\gamma Z}^{V}$ correction. Included in this analysis will be a discussion of the uncertainties which arise, particular attention will be paid to the models used by Gorchtein et al. in Ref. [110] since this is where the largest quoted uncertainty comes from. Following this we present the AJM model and the method used to construct these structure functions.

The aim ultimately will be to understand why the uncertainties are as large or as small as they are given and to use phenomenological information such as parton distribution functions - which are well understood - to constrain these uncertainties. Furthermore, as there is new data on PV electrondeuteron scattering, we will be able to test the reliability of those constrained structure functions. By using a consistent approach to the construction of the interference structure functions we hope to minimise any model dependence which may affect the final value of $\square_{\gamma Z}^{V}$. This work also provides motivation for further experimental efforts in studying these important phenomena. Should the structure functions be measured, a much more precise value of the $\square_{\gamma Z}$ correction would be possible, improving the theoretical determination of $\sin ^{2} \theta_{W}$ and therefore providing even more stringent tests of the SM. 


\section{Structure functions}

Quantum chromodynamics is responsible for some of the most complex and interesting phenomena observed in nuclear physics. Twoof which stand out in particular are confinement and asymptotic freedom. The structure of the colour $S U(3)$ gauge group means that as the energy scale increases, the quarkgluon coupling vanishes. This is asymptotic freedom and it results in hadrons appearing to consist of essentially free, point-like particles at large momenta. At the other end of the scale, confinement occurs. The quark-gluon coupling grows with decreasing energy - equivalent to large distances - causing the potential between quarks to increase. At large separation, the energy required to break the bond between quarks is sufficient to create a $q \bar{q}$ pair out of the vacuum. Consequently, quarks and gluons cannot be observed in isolation, but only in the form of bound, 'colour-singlet' states. Figure 4.1 illustrates the behaviour of the strong coupling constant, $\alpha_{s}$, as a function of momentum transfer.

A result of these properties of $\alpha_{s}$, is that it is difficult to study the internal structure of hadrons since perturbation theory can only be used at large energies. Below these, the series fails to converge. ${ }^{1}$ One particularly useful tool for understanding the structure of the nucleon which spans both the perturbative and nonperturbative regimes are structure functions. First measured $[115,116]$ in the late 1960s, these functions generalise the form factors found from elastic scattering. In fact, the results from these SLAC experiments were essential in the modern acceptance of QCD as they showed the structure functions' independence of momentum transfer $Q^{2}$ for large $Q^{2}$-a direct manifestation of asymptotic freedom.

In this chapter we introduce the formalism of structure functions and their related phenomena. Following a discussion of the deep inelastic scattering region, we describe the parton model before looking at some general parametrisations used in the Regge region. For the purposes of this thesis, a robust description of both the electromagnetic $(\gamma \gamma)$ and interference $(\gamma Z)$ structure functions is needed over all kinematic regions. Having reviewed those

\footnotetext{
${ }^{1}$ The boundary between the perturbative and nonperturbative regions is somewhat loosely defined and later discussions in Chapter 8 on the moments of structure function will touch on this issue.
} 


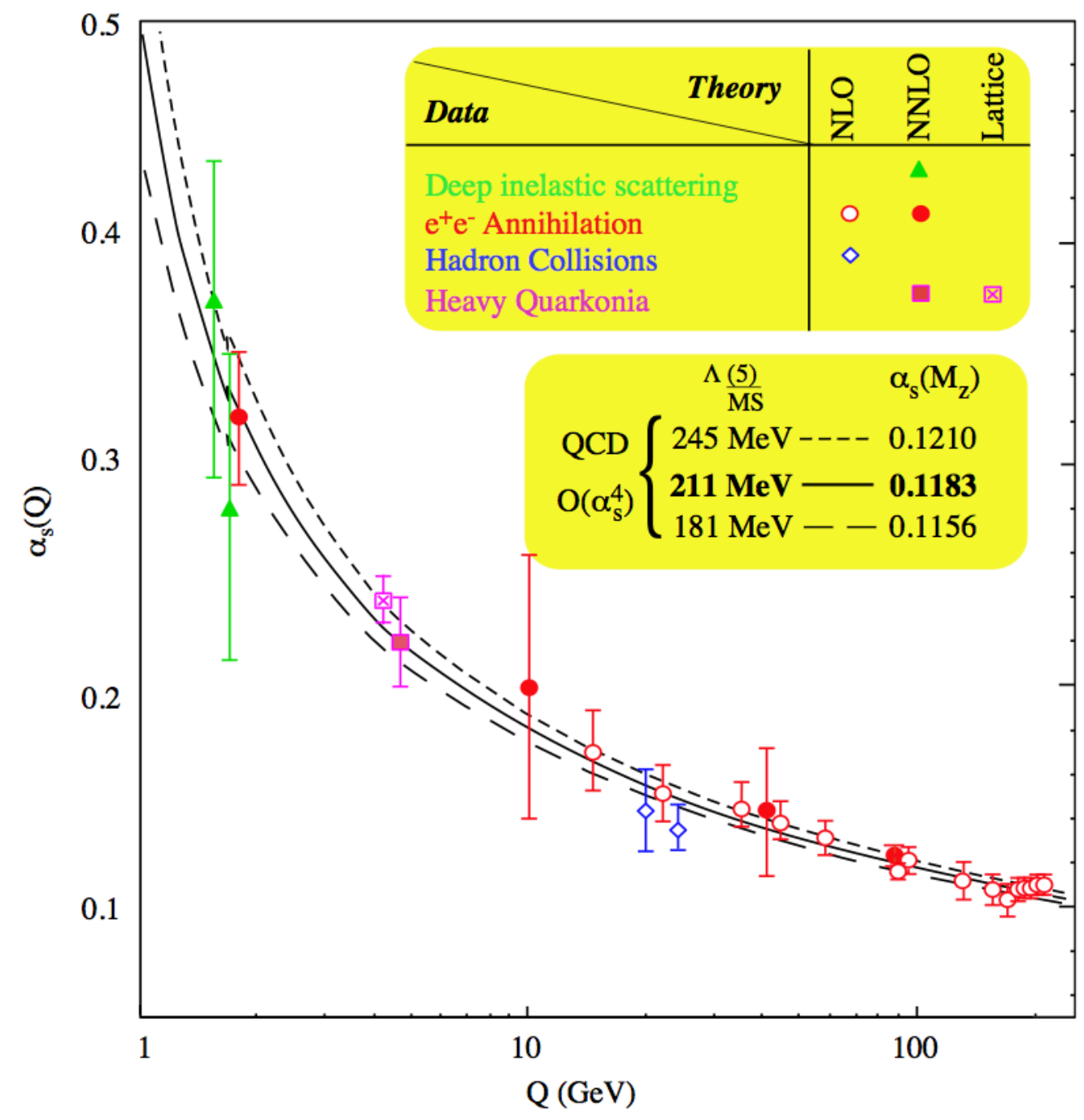

Figure 4.1: The strong coupling, $\alpha_{\mathrm{s}}$, as a function of momentum transfer [117]. The plotted points includes data with next-to-leading order (NLO) and nextto-next-to-leading (NNLO) theoretical corrections. The three curves represent alternative choices of the QCD scale parameter $\Lambda$. 
models previously used in literature we proceed with an account of the constrained Adelaide-Jefferson Lab-Manitoba model in Chapter 5.

\subsection{Deep inelastic scattering}

The physical process we are concerned with here is shown in Fig. 4.2. In inclusive scattering, a lepton (for example) with four-momentum $k$ scatters off a nucleon target. It transfers momentum $q$, before entering the experiment's detectors. The resulting hadronic products, denoted $X$, are left unidentified. (Exclusive scattering occurs when all the final products are known, while in semi-exclusive scattering some are and some are not.) In such an event there are a number of frequently used kinematic quantities which we list together here. Unless otherwise stated, we will be working in the target reference frame:

$\theta$ : scattering angle of the lepton relative to the incoming beam.

$M$ : mass of the proton.

$E$ : energy of the incoming lepton.

$E^{\prime}$ : energy of the outgoing lepton.

$\nu=\frac{p \cdot q}{M}=E-E^{\prime}$ : energy transfer of the electron to the target.

$Q^{2}=-q^{2}$ : momentum transfer of the electron.

$W^{2}=M^{2}+2 p \cdot q-Q^{2}$ : invariant mass squared of the final system.

$x=\frac{Q^{2}}{2 M \nu}$ : fraction of the nucleon's momentum carried by the parton.

$y=\frac{p \cdot q}{p \cdot k}=\frac{\nu}{E}$ : fraction of the energy lost by the lepton.

For the deep inelastic scattering event of Fig. 4.2 the cross section is given by,

$$
\frac{d^{2} \sigma}{d \Omega d E^{\prime}}=\frac{\alpha^{2}}{Q^{4}} \frac{E^{\prime}}{E} \sum_{j} \eta_{j} L_{j}^{\mu \nu} W_{\mu \nu}^{j}
$$

where $\Omega$ is the solid angle, $\alpha$ is the fine structure constant, $L_{\mu \nu}$ is leptonic tensor, and $W^{\mu \nu}$ the hadronic analogue. Note that we have already seen these two tensors in the specific case where $\gamma-Z$ interference arises (cf. Eqs. (3.173.19) and the surrounding discussion). Although we are primarily interested in neutral current processes in this thesis, for completeness, we also included the charge current term for interactions involving the $W^{ \pm}$. The sum in Eq. (4.1) is thus over $j=\gamma, W, Z$ and $\gamma Z$ where the coefficients $\eta_{j}$ are,

$$
\begin{array}{ll}
\eta_{\gamma}=1, & \eta_{\gamma Z}=\left(\frac{G_{F} M_{Z}^{2}}{2 \sqrt{2} \pi \alpha}\right)\left(\frac{Q^{2}}{Q^{2}+M_{Z}^{2}}\right), \\
\eta_{Z}=\eta_{\gamma Z}^{2}, & \eta_{W}=\frac{1}{2}\left(\frac{G_{F} M_{W}^{2}}{4 \pi \alpha} \frac{Q^{2}}{Q^{2}+M_{W}^{2}}\right)^{2} .
\end{array}
$$




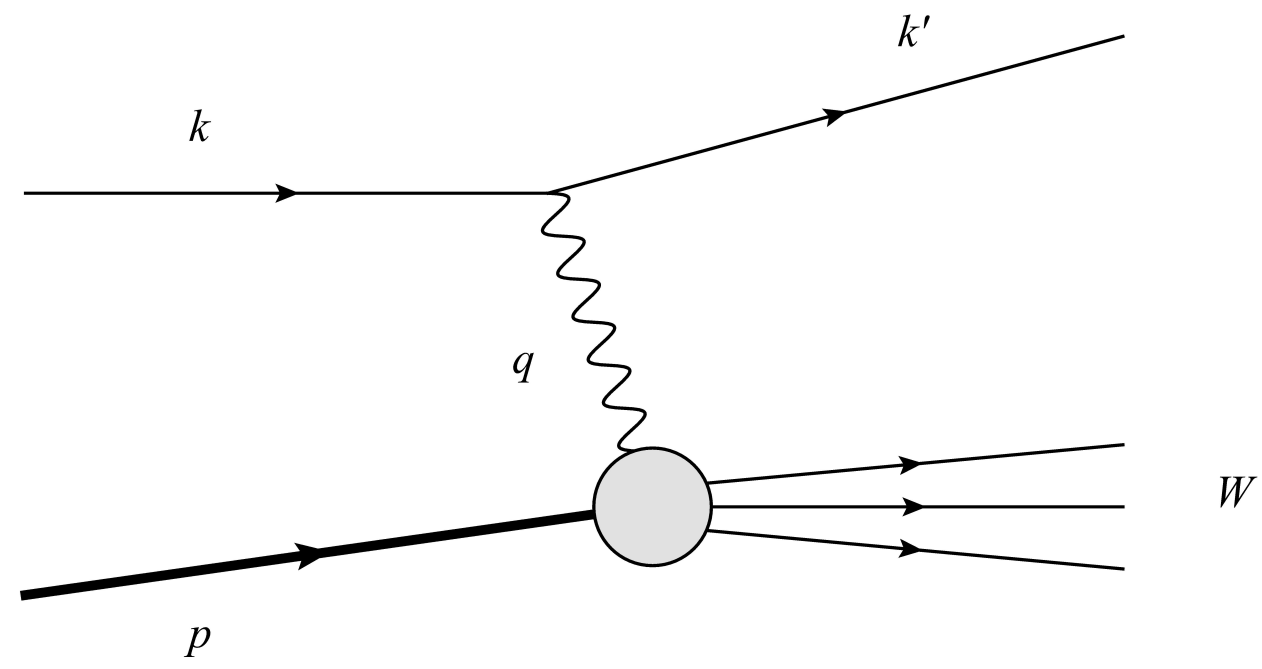

Figure 4.2: Deep inelastic $l N \rightarrow l^{\prime} X$ scattering process.

Depending on the mediating boson(s) involved, the leptonic tensor is given by,

$$
\begin{aligned}
L_{\mu \nu}^{\gamma} & =2\left(k_{\mu} k_{\nu}^{\prime}-k \cdot k^{\prime} g_{\mu \nu}-i \lambda \epsilon_{\mu \nu \alpha \beta} k^{\alpha} k^{\prime \beta}\right), \\
L_{\mu \nu}^{\gamma Z} & =\left(g_{V}^{e}+e \lambda g_{A}^{e}\right) L_{\mu \nu}^{\gamma}, \\
L_{\mu \nu}^{Z} & =\left(g_{V}^{e}+e \lambda g_{A}^{e}\right)^{2} L_{\mu \nu}^{\gamma}, \\
L_{\mu \nu}^{W} & =(1+e \lambda)^{2} L_{\mu \nu}^{\gamma},
\end{aligned}
$$

where $g_{V}^{e}$ and $g_{A}^{e}$ are the vector and axial-vector couplings from earlier. We note that here and elsewhere we follow the Particle Data Group (PDG) [3] conventions.

The hadronic tensor may be written as,

$$
\begin{aligned}
W_{j}^{\mu \nu}(p, q)=\frac{1}{2 M} & \sum_{X}\left\langle N(p)\left|J_{j}^{\mu}(0)\right| X\left(p_{X}\right)\right\rangle\left\langle X\left(p_{X}\right)\left|J_{j}^{\nu}(0)\right| N(p)\right\rangle \\
& \times(2 \pi)^{3} \delta^{(4)}\left(q+p-p_{X}\right),
\end{aligned}
$$

where,

$$
J_{j=\gamma}^{\mu}=\frac{2}{3} \bar{u} \gamma^{\mu} u-\frac{1}{3}\left(\bar{d} \gamma^{\mu} d+\bar{s} \gamma^{\mu} s\right)
$$

is the electromagnetic quark current,

$$
\begin{aligned}
J_{j=Z}^{\mu}=\bar{u} \gamma^{\mu} & \left(\frac{1}{2}-\frac{4}{3} \sin ^{2} \theta_{W}-\frac{1}{2} \gamma_{5}\right) u \\
& +\bar{d} \gamma^{\mu}\left(-\frac{1}{2}+\frac{2}{3} \sin ^{2} \theta_{W}+\frac{1}{2} \gamma_{5}\right) d+(d \rightarrow s)
\end{aligned}
$$

the weak quark current and we have dropped the larger mass quarks $c, b$ and $t$. Equation (4.6) is in fact equivalent to Eq. (2.5), only here the quark fields have 
been included explicitly. Using completeness $\left(\Sigma_{X}|X\rangle\langle X|=1\right)$ the hadronic tensor becomes,

$$
\begin{aligned}
W_{j}^{\mu \nu}(p, q) & =\frac{1}{4 \pi M} \int d^{4} z e^{i q \cdot z}\left\langle N(p)\left|J_{j}^{\mu}(z) J_{j}^{\nu}(0)\right| N(p)\right\rangle \\
& =\frac{1}{4 \pi M} \int d^{4} z e^{i q \cdot z}\left\langle N(p)\left|\left[J_{j}^{\mu}(z), J_{j}^{\nu}(0)\right]\right| N(p)\right\rangle .
\end{aligned}
$$

The constraints of Lorentz and gauge invariance require that the hadronic tensor be proportional to $g_{\mu \nu}, p_{\mu}$ and $q_{\mu}$, thus in general,

$$
\begin{aligned}
W_{\mu \nu} & =\left(-g_{\mu \nu}+\frac{q_{\mu} q_{\nu}}{q^{2}}\right) F_{1}\left(x, Q^{2}\right) \\
& +\frac{1}{p \cdot q}\left(p_{\mu}-\frac{p \cdot q}{q^{2}} q_{\mu}\right)\left(p_{\nu}-\frac{p \cdot q}{q^{2}} q_{\nu}\right) F_{2}\left(x, Q^{2}\right) \\
& -i \epsilon^{\mu \nu \lambda \rho} \frac{q_{\lambda} p_{\rho}}{2 p \cdot q} F_{3}\left(x, Q^{2}\right) \\
& +i \epsilon^{\mu \nu \lambda \rho} \frac{q_{\lambda}}{p \cdot q}\left(S_{\rho} g_{1}\left(x, Q^{2}\right)+\frac{p \cdot q S_{\rho}-S \cdot q p_{\rho}}{p \cdot q} g_{2}\left(x, Q^{2}\right)\right)
\end{aligned}
$$

where $g^{\mu \nu}$ is the spacetime metric, $\epsilon^{\mu \nu \lambda \rho}$ is the anti-symmetric tensor and $g_{i}$ the polarised structure functions. The unpolarised structure functions are denoted $F_{i}$. There is no $F_{3}\left(x, Q^{2}\right)$ for the electromagnetic case as the photon has only a vector coupling to the electron. Since the Bjorken scaling variable, $x$, can be written,

$$
\begin{aligned}
x & =\frac{Q^{2}}{2 M \nu}=\frac{Q^{2}}{2 p \cdot q} \\
& =\frac{Q^{2}}{W^{2}-M^{2}+Q^{2}},
\end{aligned}
$$

the structure functions may alternatively be written as a function of $W^{2}$ and $Q^{2}$.

For later reference it will be useful to write the structure functions $F_{i}$ in terms of the transverse $\left(\sigma_{T}\right)$ and longitudinal $\left(\sigma_{L}\right)$ cross sections:

$$
\begin{aligned}
& F_{1}\left(W^{2}, Q^{2}\right)=\left(\frac{W^{2}-M^{2}}{8 \pi^{2} \alpha}\right) \sigma_{T}\left(W^{2}, Q^{2}\right) \\
& F_{2}\left(W^{2}, Q^{2}\right)=\left(\frac{W^{2}-M^{2}}{8 \pi^{2} \alpha}\right) \frac{\nu}{M\left(1+\nu^{2} / Q^{2}\right)}\left[\sigma_{T}\left(W^{2}, Q^{2}\right)+\sigma_{L}\left(W^{2}, Q^{2}\right)\right] .
\end{aligned}
$$

These relations hold for both the electromagnetic and interference structure functions. Additionally, one may also define the longitudinal structure function as the combination of $F_{1}$ and $F_{2}$ structure functions,

$$
\begin{aligned}
F_{L} & =\left(1+\frac{Q^{2}}{\nu^{2}}\right) F_{2}-2 x F_{1}, \\
& =\left(1+\frac{4 x^{2} M^{2}}{Q^{2}}\right) F_{2}-2 x F_{1} .
\end{aligned}
$$




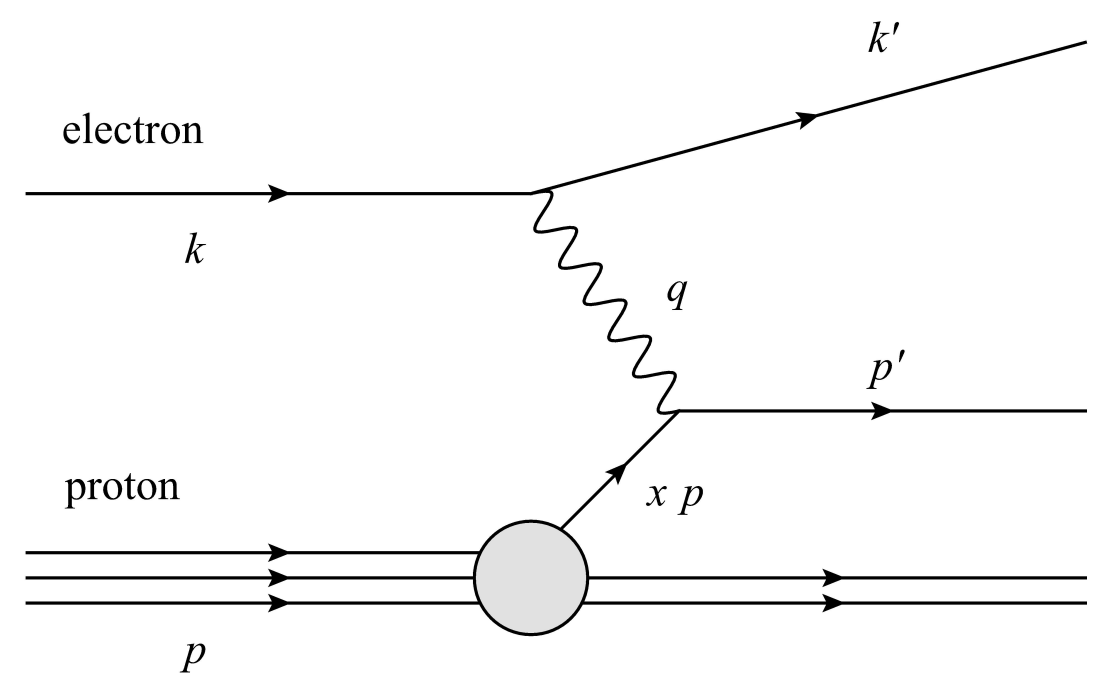

parton

Figure 4.3: Deep inelastic scattering in the quark-parton model.

Thus Eq. (4.1) may be written in terms of structure functions as,

$$
\frac{d^{2} \sigma}{d \Omega d E^{\prime}}=\frac{\alpha^{2}}{4 E^{2} \sin ^{4} \frac{\theta}{2}}\left(\frac{2}{M} \sin ^{2} \frac{\theta}{2} F_{1}\left(W^{2}, Q^{2}\right)+\frac{1}{\nu} \cos ^{2} \frac{\theta}{2} F_{2}\left(W^{2}, Q^{2}\right)\right),
$$

where, $\theta$ is the laboratory scattering angle.

The physical interpretation of the structure functions depend on the kinematic region in which the scattering event is taking place. In the DIS region where the strong coupling constant is small, the quark-parton model may be used. This is not the case for the lower $Q^{2}$ region, however, where large $\alpha_{s}$ prevents cross sections from being calculated using perturbation theory. Instead, phenomenological models are employed. Given that resonance structure is present at low $Q^{2}$, these parametrisations must necessarily include such structure also.

\subsection{Quark-parton model}

At large momentum transfer and invariant mass (DIS region), the nucleon may be modelled as a collection of quasi-free, point-like quarks and gluonsgenerically referred to as partons [118]. In this picture, when an electron collides with a proton, the virtual photon interacts with the partons individually. In order to calculate the total cross section for the electron-proton scattering process, one must sum over the cross section for each parton. Moreover, since the density of partons in the proton differs according to their flavour, and each parton carries distinct fractions of the total momentum, the cross sections for the quarks and gluons will be a function of both their flavour and their momentum fraction $x$. In Fig. 4.3 we show what takes place diagrammatically.

The structure functions $F_{i}$ can be expressed in terms of these number densities, or 'parton distribution functions'. For the neutral current structure 
functions relevant to this work, we have,

$$
\begin{aligned}
& {\left[F_{2}^{\gamma \gamma}, F_{2}^{\gamma Z}\right]=x \sum_{q}\left[e_{q}^{2}, 2 e_{q} g_{V}^{q}\right] \cdot q\left(x, Q^{2}\right),} \\
& {\left[F_{3}^{\gamma \gamma}, F_{3}^{\gamma Z}\right]=x \sum_{q}\left[0,2 e_{q} g_{A}^{q}\right] \cdot q\left(x, Q^{2}\right),}
\end{aligned}
$$

where $q=u, d, s, c, b, t$ are the quark distribution functions. Because the cross section for the longitudinally polarized photon, $\sigma_{L}$, disappears at high energies, from Eqs. (4.10) and (4.11) it is clear that the $F_{1}$ and $F_{2}$ are now both proportional simply to the cross section of the transverse photon. Thus,

$$
F_{2}(x)=2 x F_{1}(x)
$$

and by substituting Eq. (4.14), into Eq. (4.15) we see that the $F_{1}^{i}$ are given as,

$$
\left[F_{1}^{\gamma \gamma}, F_{1}^{\gamma Z}\right]=\frac{1}{2} \sum_{q}\left[e_{q}^{2}, 2 e_{q} g_{V}^{q}\right] \cdot q\left(x, Q^{2}\right)
$$

Equation (4.15) is known as the Callan-Gross relation [119].

In Fig. 4.4 we show an example of the parton densities for the quarks, antiquarks and gluons in the proton at a particular $Q^{2}$.

\subsection{Modelling structure functions}

While the quark-parton model works well in the DIS region, for low momentum transfer $\left(Q^{2} \lesssim 2.5 \mathrm{GeV}^{2}\right)$ alternative models are needed. In fact, the low- $Q^{2}$ region may itself be divided further into a resonance and Regge part. The rest of this section will describe some of the more commonly used models for the electromagnetic structure functions. These form the basis of later constructions of the interference structure functions.

\section{Christy-Bosted parametrisation}

In the resonance region, Christy and Bosted (CB) have parametrised the precision electromagnetic inclusive cross section data from Jefferson Lab's E94110 experiment [113]. For the proton this is described in Ref. [121], and the deuteron, Ref. [122]. When modelling the structure functions, the transverse and longitudinal cross sections may be separated into a resonance piece and a smooth, underlying nonresonance background,

$$
\sigma_{T, L}=\sigma_{T, L}^{(\mathrm{res})}+\sigma_{T, L}^{(\mathrm{bgd})}
$$

The $\sigma_{T, L}^{\text {(res) }}$ term involves a sum over the seven dominant resonances: $P_{33}(1232), P_{11}(1440), D_{13}(1520), S_{11}(1535), S_{15}(1650), F_{15}(1680)$ and an additional $l=3$ state with mass $1934 \mathrm{MeV}$. The shape of the background piece 


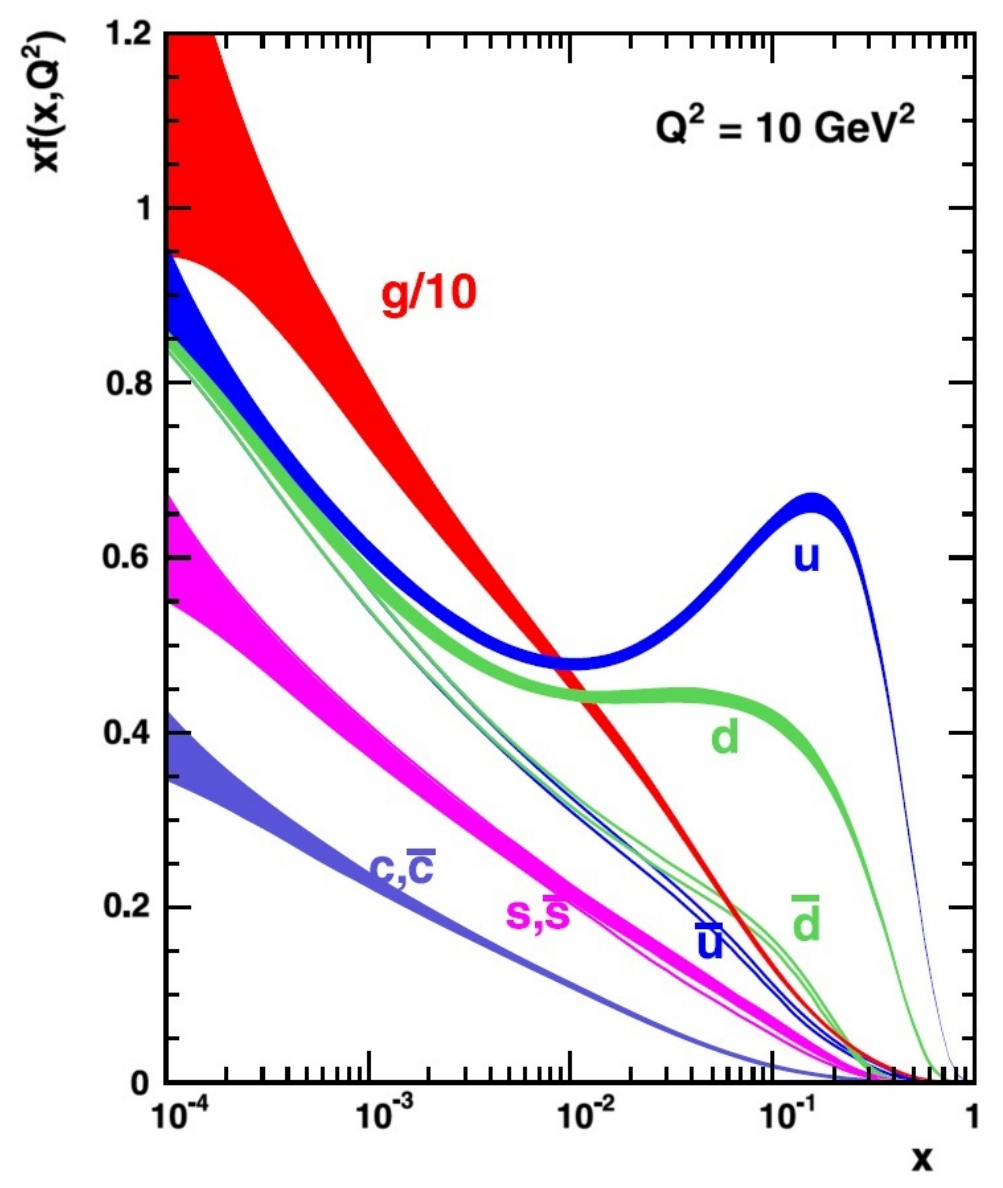

Figure 4.4: Parton distribution functions of the proton at $Q^{2}=10 \mathrm{GeV}^{2}[120]$.

was found by fitting to the scattering data. It is important to point out that this separation into a resonance and nonresonant part is inherently model dependent since experiments are only able to determine the total cross section.

The Christy-Bosted proton structure functions are in good agreement with the data, with almost all the experimental points differing from the fit by less than 5\% [121]. Although the deuteron and neutron structure functions are not quite so precise, still less than $4 \%$ of the points deviate by more than $10 \%$ [122]. Kinematically, the proton fit is valid for $0 \leq Q^{2}<8 \mathrm{GeV}^{2}$ and $1.1<W<3.1 \mathrm{GeV}$, with similar bounds for the deuteron. Since this covers a significant portion of the DIS region, it allows for valuable comparisons between the PDFs and CB's parametrisation.

As a result of its accuracy, the $\mathrm{CB}$ fit has been used as the basis for most of the other models utilised. In their construction of the $\gamma Z$ structure functions, Gorchtein et al. (GHRM) use CB's resonance cross section in both their 'Model I' and 'Model II'. (Some modifications to the parameters were needed to better match their background, however [110].) Carlson and Rislow $(\mathrm{CR})$ on the other hand, use the complete cross section for their low $Q^{2}$, low 


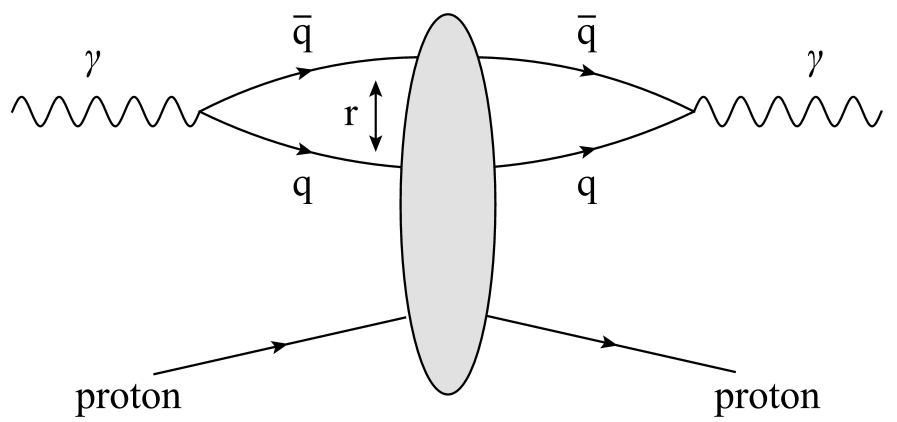

Figure 4.5: The interaction of a virtual photon with a proton in the CDP model.

$W^{2}$ region $[109,123]$. In contrast Sibirtsev et al. (SBMT) perform their own fit of the data, incorporating the four resonances $P_{33}(1232), D_{13}(1520), F_{15}(1680)$ and $F_{37}(1950)$ [108]. They also obtain a good description of the data.

\section{Colour dipole model}

Initially formulated $[124,125,126]$ in the 1990 s, the dipole picture provides a valid description of $\gamma p$ scattering ranging from $Q^{2} \approx 0.25 \mathrm{GeV}^{2}$ all the way up to $Q^{2}$ of order hundreds of $\mathrm{GeV}^{2}$ [127]. The $W^{2}$ region extends even further, reaching as high as $\sim 60000 \mathrm{GeV}^{2}$ [127]. Scattering in the colour dipole (CDP) model may be thought of as taking place in three distinct stages; first, the virtual photon fluctuates into a $q \bar{q}$ pair, then the quark-antiquark dipole interacts with the proton, before finally it reforms into a virtual photon again. This process is illustrated in Fig. 4.5.

The cross section for the $\gamma^{*} p$ interaction may be written as [128],

$$
\sigma_{\gamma^{*} p}^{T, L}=\int d z \int d^{2} r\left|\psi_{\gamma}^{T, L}\left(r, z, Q^{2}\right)\right|^{2} \sigma_{(q \bar{q}) p}(r, s)
$$

where $\psi_{\gamma}^{T, L}\left(r, z, Q^{2}\right)$ is the transition amplitude for $\gamma^{*} \rightarrow q \bar{q}$ for transversely and longitudinally polarised photons. (The asterisk in $\gamma^{*}$ refers to the fact that we are considering only virtual photons.) The forward direction is given by $z$, while $r$ is the transverse distance between the $q \bar{q}$ pair and $\sigma_{(q \bar{q}) p}(r, s)$ is the cross section for the interaction between the dipole and the proton. Note that as the transverse spacing between quarks goes to zero, so does the cross section for the $(q \bar{q}) p$ reaction [127].

GHRM [110] used a hybrid model of the CDP with the generalised vector dominance model [127, 129] for the background part of the Model I electromagnetic cross section. This was then combined with the resonance piece using Eq. (4.17) to obtain the total cross section. 


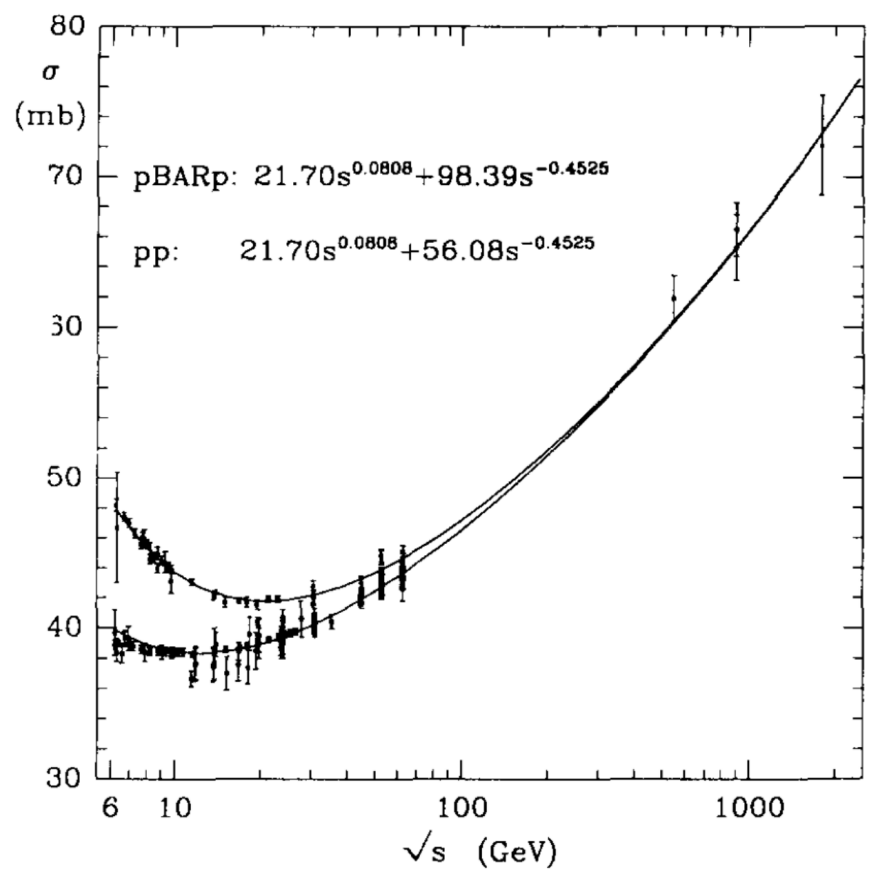

Figure 4.6: A Regge fit to $p p$ and $\bar{p} p$ cross sections from Ref. [132].

\section{Regge models}

With decreasing energies, the increasing size of the strong coupling, $\alpha_{s}$, prevents perturbation theory from being a viable technique for describing strong interactions. Historically, phenomenological models such as Regge theory or S-matrix theory were used as an alternative way of gaining physical insight into the reactions that took place. Provided one remained in the appropriate kinematic region, these models gave a good description of the data. As Regge parametrisations underlie a number of the models discussed in this work, we give a brief description of the relevant features here. For more complete accounts we refer the reader to Refs. [130, 131].

In Regge theory the cross sections are given generally as [132],

$$
\sigma_{\mathrm{tot}}=A s^{\epsilon}+B s^{-\eta}
$$

where $\sigma_{\text {tot }}$ is the total cross section and $s$ centre-of-mass energy squared. The first term on the right-hand side originates from pomeron exchange, while the second comes from exchanging the $\rho, \omega$ and $f$ mesons. The rest of the parameters, $A, B, \epsilon$ and $\eta$ are all found by fitting to data. (Note that although the values for $\epsilon$ and $\eta$ are given here as constants in actual fact they will vary slowly with $s$ [132].) An example of a Regge fit to data from Ref. [132] is shown in Fig.4.6 giving a good description of data over a large range of $\sqrt{s}$.

The authors of Ref. [110] use a similar Regge parametrisation, in combination with the vector meson dominance model, to give the background part of their electromagnetic cross section of Model II. This combination is also 
used in our model. Sibirtsev et al. on the other hand, base their background piece on the Regge parametrisation of Ref. [133].

\section{Vector meson dominance model}

Strongly related to the dipole model discussed previously, the vector meson dominance model (VMD) approximates the photon state as the sum of vector mesons which carry the same quantum numbers,

$$
|\gamma\rangle=C_{0}\left|\gamma_{0}\right\rangle+\sum_{V} \frac{e}{f_{V}}|V\rangle
$$

where $f_{V}$ are the decay constants and the sum is over the $\rho, \omega$ and $\phi$ mesons. The $\left|\gamma_{0}\right\rangle$ term represents the electromagnetic part of the photon, while hadronic interactions are given by the $\frac{e}{f_{V}}|V\rangle$ terms. This model leads to an expression for the virtual transverse cross section of the form [134, 135],

$$
\sigma_{T}=\sum_{V} \frac{4 \pi \alpha}{f_{V}^{2}}\left(\frac{m_{V}^{2}}{Q^{2}+m_{V}^{2}}\right)^{2} \sigma_{V p}
$$

where $m_{V}$ is the mass of the vector meson $V$ and $\sigma_{V p}$ is the cross section for the $V p$ interaction.

However, there is an issue since the above expression accounts for only $80 \%$ of the experimental cross section. Because of this, another 'continuum' piece,

$$
\sigma_{T}^{C}\left(W^{2}, Q^{2}\right)=\int_{m_{0}^{2}} d m^{2} d m^{\prime 2} \frac{\tilde{\rho}_{T, L}\left(W^{2}, m^{2}, m^{\prime 2}\right) m^{2} m^{\prime 2}}{\left(m^{2}+Q^{2}\right)\left(m^{\prime 2}+Q^{2}\right)},
$$

is added to form the so-called generalised vector meson dominance model. This extra factor accounts for the higher mass hadronic contributions to the photon. In practice, the off-diagonal $m \neq m^{\prime}$ terms are dropped leaving,

$$
\sigma_{T}^{C}\left(W^{2}, Q^{2}\right)=\int_{m_{0}^{2}} d m^{2} \frac{\tilde{\rho}_{T, L}\left(W^{2}, m^{2}\right) m^{4}}{\left(m^{2}+Q^{2}\right)^{2}} .
$$

The full expressions for the transverse and longitudinal cross sections then become [134, 135],

$$
\begin{aligned}
& \sigma_{T}^{\mathrm{VMD}}=\sigma_{\gamma N} {\left[\sum_{V} r_{V} \frac{1}{\left(1+Q^{2} / m_{V}^{2}\right)^{2}}+r_{C} \frac{1}{1+Q^{2} / m_{0}^{2}}\right], } \\
& \sigma_{L}^{\mathrm{VMD}}=\sigma_{\gamma N}\left[\sum_{V} r_{V} \xi_{V} \frac{Q^{2} / m_{V}^{2}}{\left(1+Q^{2} / m_{V}^{2}\right)^{2}}\right. \\
& \\
&\left.+r_{C} \xi_{C}\left(\frac{m_{0}^{2}}{Q^{2}} \ln \left(1+Q^{2} / m_{0}^{2}\right)-\frac{1}{1+Q^{2} / m_{0}^{2}}\right)\right],
\end{aligned}
$$


Table 4.1: Summary of the vector hadron correction results presented in the literature and the models on which they are based.

\begin{tabular}{lclc}
\hline \hline Authors & $\Re e \square_{\gamma Z}^{V}\left(\times 10^{-3}\right)$ & \multicolumn{1}{c}{ Models } & Ref. \\
\hline GHRM & $5.46 \pm 2.0$ & CB, Colour dipole, VMD & {$[110]$} \\
SBMT & $4.7_{-0.4}^{+1.1}$ & Capella, Regge & {$[108]$} \\
CR & $5.7 \pm 0.9$ & CB, Capella, PDFs & {$[109]$} \\
\hline \hline
\end{tabular}

where $r_{V} \sim 1 / f_{V}^{2}$ gives the fractional contribution from each meson ${ }^{2}$ and

$$
r_{C}=1-\sum_{V} r_{V}
$$

The cross section $\sigma_{\gamma N}$ is for the $\gamma-N$ interaction and takes the Regge form of Eq. (4.19) i.e. it is proportional to $A s^{\epsilon}+B s^{-\eta}$, while $\xi_{V}$ and $\xi_{C}$ account for any difference in the transverse and longitudinal components of the vector mesons. In most cases, however, these are simply taken to be equal, $\xi_{V}=\xi_{C}$.

In constructing the interference structure functions the continuum piece in Eqs. (4.24) and (4.25) plays a critical role in quantifying the size of the cross sections' uncertainties. The VMD model is used for the background term in both Model II of Ref. [110] and in our model. (Strictly speaking, this should be referred to as the generalised vector meson dominance model, however, in order to avoid confusion, we follow the convention of Gorchtein et al. and simply refer to it as the vector meson dominance model, or 'VMD'.)

\section{4 $\gamma Z$ interference structure functions}

In general, constructing the $\gamma Z$ structure functions from their electromagnetic counterparts involves first transforming the resonance piece, followed by a separate modification to the background part. In the next section we discuss the interference structure functions models which have been used and the uncertainties they generate. A summary of the results for the $\square_{\gamma Z}^{V}$ correction found using these parametrisations is given in Table. 4.1.

\section{Gorchtein, Horowitz and Ramsey-Musolf}

Each resonance in the $\mathrm{CB}$ parametrisation is modified by a ratio that incorporates the differences between the electromagnetic and weak neutral amplitudes. Beginning with the matrix elements of the proton, isospin symmetry allows the vector part of the $Z$ current to be written in terms of the electromagnetic current as follows,

$$
\left\langle R\left|J_{Z}^{\mu}\right| p\right\rangle=\left(1-4 \sin ^{2} \theta_{W}\right)\left\langle R\left|J_{\gamma}^{\mu}\right| p\right\rangle-\left\langle R\left|J_{\gamma}^{\mu}\right| n\right\rangle,
$$

\footnotetext{
${ }^{2}$ Although there appears to be a $1 / Q^{2}$ dependence in the second term of Eq. (4.25), by expanding out the logarithm one can check that the cross section vanishes as $Q^{2} \rightarrow 0$.
} 
with $R$ referring to the specific resonance. Although there is also a contribution from the strange quarks, for most purposes considered here, it is negligible. Since the cross sections are proportional to the square of the amplitudes, the transverse $\gamma \gamma / \gamma Z$ ratio may be defined as [110],

$$
\xi_{R} \equiv \frac{\sigma_{T, R}^{\gamma Z}}{\sigma_{T, R}^{\gamma \gamma}}=\left(1-4 \sin ^{2} \theta_{W}\right)-y_{R}
$$

where

$$
y_{R}=\frac{A_{R, \frac{1}{2}}^{p} A_{R, \frac{1}{2}}^{n^{*}}+A_{R, \frac{3}{2}}^{p} A_{R, \frac{3}{2}}^{n^{*}}}{\left|A_{R, \frac{1}{2}}^{p}\right|^{2}+\left|A_{R, \frac{3}{2}}^{p}\right|^{2}}
$$

and $A_{R, \lambda}^{N}$ is the transition amplitude from the proton or neutron to a resonance with helicity $\lambda=\frac{1}{2}$ or $\frac{3}{2}$. The uncertainties for the $y_{R}$ ratios given by the PDG [136] and shown in Chapter 5, are considered by GHRM to be large enough to account for any $Q^{2}$ dependence which is therefore set to zero. GHRM also take the longitudinal and transverse ratios to be equal for both Models I and II.

The construction of the $\gamma Z$ background follows in a similar manner to the resonance section. As before, the electromagnetic part needs to be modified by the ratio $\sigma_{T, L}^{\gamma Z} / \sigma_{T, L}^{\gamma \gamma}$. In Model II of Ref. [110] the denominator is given by the VMD, while for the numerator, each term is scaled by the ratio $\kappa_{V}$ of weak and electric charges,

$$
\sigma_{T, L}^{\gamma Z(V)}=\kappa_{V} \sigma_{T, L}^{\gamma \gamma(V)}
$$

where

$$
\begin{aligned}
\kappa_{\rho} & =2-4 \sin ^{2} \theta_{W}, \\
\kappa_{\omega} & =-4 \sin ^{2} \theta_{W}, \\
\kappa_{\phi} & =3-4 \sin ^{2} \theta_{W} .
\end{aligned}
$$

From Eqs. (4.30) and (4.31) the total ratio of the $\gamma Z$ and $\gamma \gamma$ cross sections may be written,

$$
\frac{\sigma_{T, L}^{\gamma Z}}{\sigma_{T, L}^{\gamma \gamma}}=\frac{\kappa_{\rho}+\kappa_{\omega} R_{\omega}^{T, L}\left(Q^{2}\right)+\kappa_{\phi} R_{\phi}^{T, L}\left(Q^{2}\right)+\kappa_{C}^{T, L} R_{C}^{T, L}\left(Q^{2}\right)}{1+R_{\omega}^{T, L}\left(Q^{2}\right)+R_{\phi}^{T, L}\left(Q^{2}\right)+R_{C}^{T, L}\left(Q^{2}\right)},
$$

where $R_{V}^{T, L}$ is the vector meson cross sections normalised by the $\rho$ meson cross section,

$$
\begin{aligned}
R_{V}^{T, L} & \equiv \frac{\sigma_{T, L}^{\gamma \gamma(V)}}{\sigma_{T, L}^{\gamma \gamma(\rho)}} \\
& =\frac{f_{\rho}^{2}}{f_{V}^{2}}\left(\frac{1+Q^{2} / m_{\rho}^{2}}{1+Q^{2} / m_{V}^{2}}\right)^{2},
\end{aligned}
$$


and the analogous ratios for the continuum pieces are,

$$
\begin{aligned}
R_{C}^{T} & =\frac{r_{C}}{r_{\rho}}\left(\frac{1+Q^{2} / m_{\rho}^{2}}{1+Q^{2} / m_{0}^{2}}\right)^{2}, \\
R_{C}^{L} & =\frac{r_{C}}{r_{\rho}}\left[\frac{m_{0}^{2}}{Q^{2}} \ln \left(1+Q^{2} / m_{0}^{2}\right)-\frac{1}{1+Q^{2} / m_{0}^{2}}\right] /\left[\frac{Q^{2} / m_{\rho}^{2}}{\left(1+Q^{2} / m_{\rho}^{2}\right)^{2}}\right],
\end{aligned}
$$

with the mass parameter $m_{0}$ set to $1.5 \mathrm{GeV}$ [110].

The ratios of the $\gamma Z$ and $\gamma \gamma$ continuum contributions are represented by the $\kappa_{C}^{T, L}$ parameters for which there is no straightforward method of prescribing values to these numbers - unlike the discrete meson ratios. As a result, GHRM equate the electromagnetic and interference continuum term and assign a 100\% uncertainty to this contribution.

Modification of the background in Model I of Ref. [110] again uses the general form of Eq. (4.32), however here, the $R_{V}^{T, L}$ are no longer functions of $Q^{2}$ and are instead given by the squares of the quark electric charges. The continuum piece is approximated by the $J / \psi$ meson [110],

$$
\{\rho: \omega: \phi: J / \psi\}=\{1: 1 / 9: 2 / 9: 8 / 9\} .
$$

In a similar manner to before, the $J / \psi$ piece is assigned a $100 \%$ uncertainty.

Although Gorchtein et al. use two different parametrisations to examine the model dependence of the $\square_{\gamma Z}$ contribution, the final quoted value is an average of the two [110]. The uncertainty is dominated by the background error which accounts for about $90 \%$ of the total uncertainty. ${ }^{3}$ Model dependence makes up for $\sim 2 \%$ of the total uncertainty, while the remainder $(\sim 8 \%)$ comes from the resonances.

\section{Sibirtsev, Blunden, Melnitchouk and Thomas}

To transform the resonances, SBMT use the SU(6) quark model wave functions to obtain an estimate for the ratio of the couplings of the isospin- $1 / 2$ resonances. Given the similarity between the weak and electromagnetic couplings, these ratios are set to one. The $3 / 2$ resonances' ratios on the other hand, are approximated as $\left(1+Q_{W}^{p}\right) \approx\left(2-4 \sin ^{2} \theta_{W}\right)$ using isospin symmetry and conservation of vector current.

Based on the approximate flavour independence of the sea quark distributions and the fact that the ratio of the sum of the electroweak couplings are almost equal [107],

$$
\begin{aligned}
\left(\sum_{q} e_{q} g_{V}^{q}\right) /\left(\sum_{q} e_{q}^{2}\right) & =2-4 \sin ^{2} \theta_{W} \\
& \approx 1
\end{aligned}
$$

\footnotetext{
${ }^{3}$ In their analysis, GHRM take the background uncertainty from the model which gives the largest error i.e., Model II.
} 
at low $x$, Sibirtsev et al. assume the electromagnetic and $\gamma Z$ structure functions to be equal in this region. For larger $x$ where this approximation no longer holds, they use the ratio,

$$
F_{i}^{\gamma Z}=\left(\frac{F_{i}^{\gamma Z}}{F_{i}^{\gamma \gamma}}\right)^{\mathrm{LT}} F_{i}^{\gamma \gamma}
$$

where LT refers to the leading twist structure functions - given, in this case, by the MRST parton distribution functions [137]. Since the electromagnetic structure functions are $\sim 40 \%$ larger than the interference function, the $F_{i}^{\gamma \gamma}$ may be considered an upper limit on $F_{i}^{\gamma Z}$.

The estimated errors on the $\square_{\gamma Z}$ correction by SBMT, arising from the uncertainties in the fit parameters [108], are significantly smaller than GHRM's [110]. Averaging the upper and lower errors in Table. 4.1 results in an error more than two and half times less than those in Ref. [110].

\section{Carlson and Rislow}

Resonances in Carlson and Rislow's structure functions are modified by a similar relation to Eq. (4.28). However, their ratio (labelled $C_{R}$ ) improves upon the above two models by including an additional $Q^{2}$ dependence which is incorporated using the MAID model of Ref. [138]. The transition amplitudes in Eq. (4.29) are calculated using the constituent quark model [109, 123].

For the background, Carlson and Rislow scale CB's background cross section by the ratio $F_{i}^{\gamma Z} / F_{i}^{\gamma \gamma}$ averaged over the high energy limit where

$$
F_{i}^{\gamma Z} / F_{i}^{\gamma \gamma}=2-4 \sin ^{2} \theta_{W}
$$

and the $\mathrm{SU}(6)$ quark limit where,

$$
F_{i}^{\gamma Z} / F_{i}^{\gamma \gamma}=5 / 3-4 \sin ^{2} \theta_{W} .
$$

In the Regge region, CR use Eq. (4.38) to modify a background given by Capella et al. [133], while the DIS region structure functions are given by the PDFs of Ref. [139].

In low- $Q^{2}$, low- $W^{2}$ region, CR assign a conservative $10 \%$ error to allow for the (small) error in CB's fit and the modifications of the resonances. The error for the background is given by the extremes of the high energy and the SU(6) quark limits. Once more the uncertainties are substantially lower than GHRM's. Although marginally larger than those found by Sibirtsev et al., CR's errors remain less than half of the estimates of Gorchtein et al. 



\section{Adelaide-Jefferson Lab-Manitoba model}

Before proceeding with the $\square_{\gamma Z}^{V}$ evaluation, we present the details of the AJM model. By combining the strongest features of previous work with the constraints available from parton distributions, we are able to construct $\gamma Z$ structure functions with significantly reduced uncertainties. Since none of the prior models employed this method, we argue that the AJM uncertainties most realistically reflect the currently available knowledge on the $F_{i}^{\gamma Z}$. Further tests provided by $e-d$ PVDIS data confirm the robustness of this model.

As for earlier $\gamma Z$ models, the AJM structure functions may be developed in two steps: the first deals with the foundational electromagnetic structure functions, and the second, with the transformation into the interference cross sections. In Section 5.1 we describe the $\gamma \gamma$ structure functions used for the entire $Q^{2}-W^{2}$ plane. This is followed by a section on the $\gamma Z$ construction. The rest of the chapter is devoted to an account of the procedure used to constrain the cross sections and will involve constraints from PDFs and PVES data, as well as a comparison of the two.

\subsection{Electromagnetic parametrisation}

In the AJM model, the parametrisation of the electromagnetic structure functions used depends on the kinematic region being studied. For the purposes of our work we follow Carlson and Rislow [109, 123, 140] and divide the $Q^{2}-W^{2}$ plane into separate kinematic areas. ${ }^{1}$ The regions are shown diagrammatically in Fig. 5.1:

Region I: Identified by the blue shading, this area is dominated by the resonance region - roughly given by the bounds $Q^{2}<2 \mathrm{GeV}^{2}$ and $W^{2}<4 \mathrm{GeV}^{2}$. (While there remains some resonance structure outside of this region, their contributions become increasingly modest.)

\footnotetext{
${ }^{1}$ Note that these divisions differ from $\mathrm{CR}$ whose resonance region is given by $W<2.5$ $\mathrm{GeV}$, the Regge region by, $Q^{2}<5 \mathrm{GeV}^{2}$ and $W>2.5 \mathrm{GeV}$ and the DIS by $Q^{2}>5 \mathrm{GeV}^{2}$ and $W>2.5 \mathrm{GeV}[109]$.
} 
The outer boundaries of this region overlap with both the Regge and DIS regimes.

Region II: This red shaded area is best described using Regge theory.

Region III: The structure functions at kinematics within this green area are given by the parton distribution functions discussed in the previous section. Although the outer boundaries of this region are infinite, in practice, we are constrained by the energy limits of the PDF parametrisations.

In addition to the above named regions, Fig. 5.1 also contains unlabelled grey $\left(Q^{2}>0 \mathrm{GeV}^{2}, M^{2}<W^{2}<\left(M+m_{\pi}\right)^{2} \mathrm{GeV}^{2}\right)$ and white $\left(Q^{2}>10 \mathrm{GeV}^{2}\right.$, $\left(M+m_{\pi}\right)^{2}<W^{2}<4 \mathrm{GeV}^{2}$ ) sections. ( $M$ is the mass of the proton, and $m_{\pi}$ the pion mass.) The former represents the elastic region, while the latter strictly speaking, is the resonance regime. However, since the resonances die off as a factor of $1 / Q^{4}$, the white region's contribution to the structure functions is negligible. The background piece in this section is also negligible. Although the various kinematic regimes have been given well defined boundaries, physically, there is clearly overlap between regions. It will be necessary later to show that the calculation of the $\square_{\gamma Z}^{V}$ correction is not dependent upon these 'hard' boundaries.

For the low- $Q^{2}$, low- $W^{2}$ region (Region I), we use Christy and Bosted's parametrisation of the electromagnetic structure functions described in Section 4.3. The limits of their parametrisation extend from $Q^{2}=0$ up to 10 $\mathrm{GeV}^{2}$ and for $W^{2}$, between $\left(M+m_{\pi}\right)^{2}$ and $9 \mathrm{GeV}^{2}$. For larger $W^{2}$, the background quickly dies off and the parametrisation is no longer a good description of the data. Although for our model we do not directly use the $Q^{2}>2.5 \mathrm{GeV}^{2}$, $W^{2}>4 \mathrm{GeV}^{2}$ section of the parametrisation - instead incorporating it into the DIS region - the fact that multiple models are valid in the same region allows for useful comparisons. Indeed, this is what allows the resonance region of the $\gamma Z$ structure functions to be constrained by the PDFs. Even though the resonances contribute to the entire Region $\mathrm{I}$, in practice, the resonance form factors are strongly suppressed as $Q^{2}$ increases. Thus beyond $Q^{2} \approx 2 \mathrm{GeV}^{2}$ these contributions are negligible.

Regge theory is valid for moderate $Q^{2}$ and up to large $W^{2}$, and thus most applicable to Region II. For this region, the VMD+Regge description of Alwall and Ingelman [134] is combined with the resonance part of CB to give the full cross section over $Q^{2}<2.5 \mathrm{GeV}^{2}$ and $W^{2}>9 \mathrm{GeV}^{2}$. Note also that the resonance parameters of $\mathrm{CB}$ have been slightly adjusted in order to better match the background of Ref. [134]. (The modified values are given in Table II of Ref. [110].) As in the large- $Q^{2}$ area above Region I, however, the contribution from the resonances will be small. In addition, we point out that this VMD+Regge parametrisation is the Model II used by GHRM, although in their case, it was extended to incorporate the entire kinematic region of the dispersion integral. 


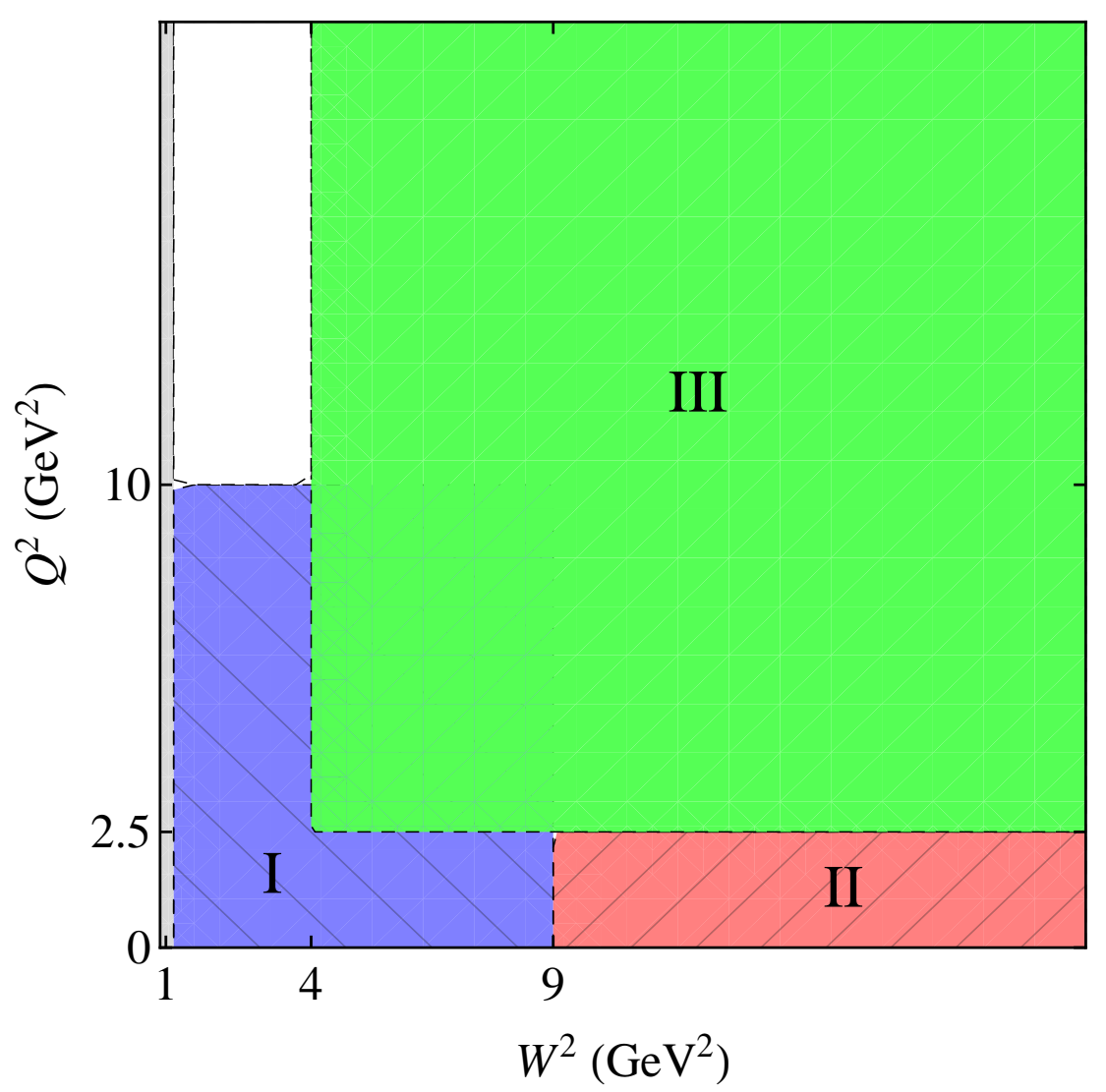

Figure 5.1: Separation of the $Q^{2}-W^{2}$ plane into the resonance, Regge and DIS regions $[90]$.

In the top half of Fig. 5.2 we show the matching at the boundaries between Regions I and II, of the CB and VMD+Regge parametrisations for several values of $Q^{2}$. It is clear from these plots that there is excellent agreement between the $\mathrm{CB}$ and VMD+Regge parametrisations. We follow CB in assigning a $5 \%$ error to the Region I electromagnetic structure functions. Comparing the VMD+Regge parametrisation with data reveals that a $5 \%$ error on these structure functions is sufficient to account for any variation between model and experiment.

Structure functions in the remaining large- $Q^{2}$, large- $W^{2}$ region (Region III) are computed from PDFs given by Alekhin et al. (ABM11) [141]. These global fits are calculated up to next-to-next-to-leading order and include both leading-twist and twist- 4 contributions. Target mass corrections were also accounted for in their constructions. In Chapter 8 we will more closely examine the size of higher-twist contributions to the moments of the structure functions, here however, we employ the full structure functions in the $\square_{\gamma Z}^{V}$ expression. Although the range of these PDFs extends further down 

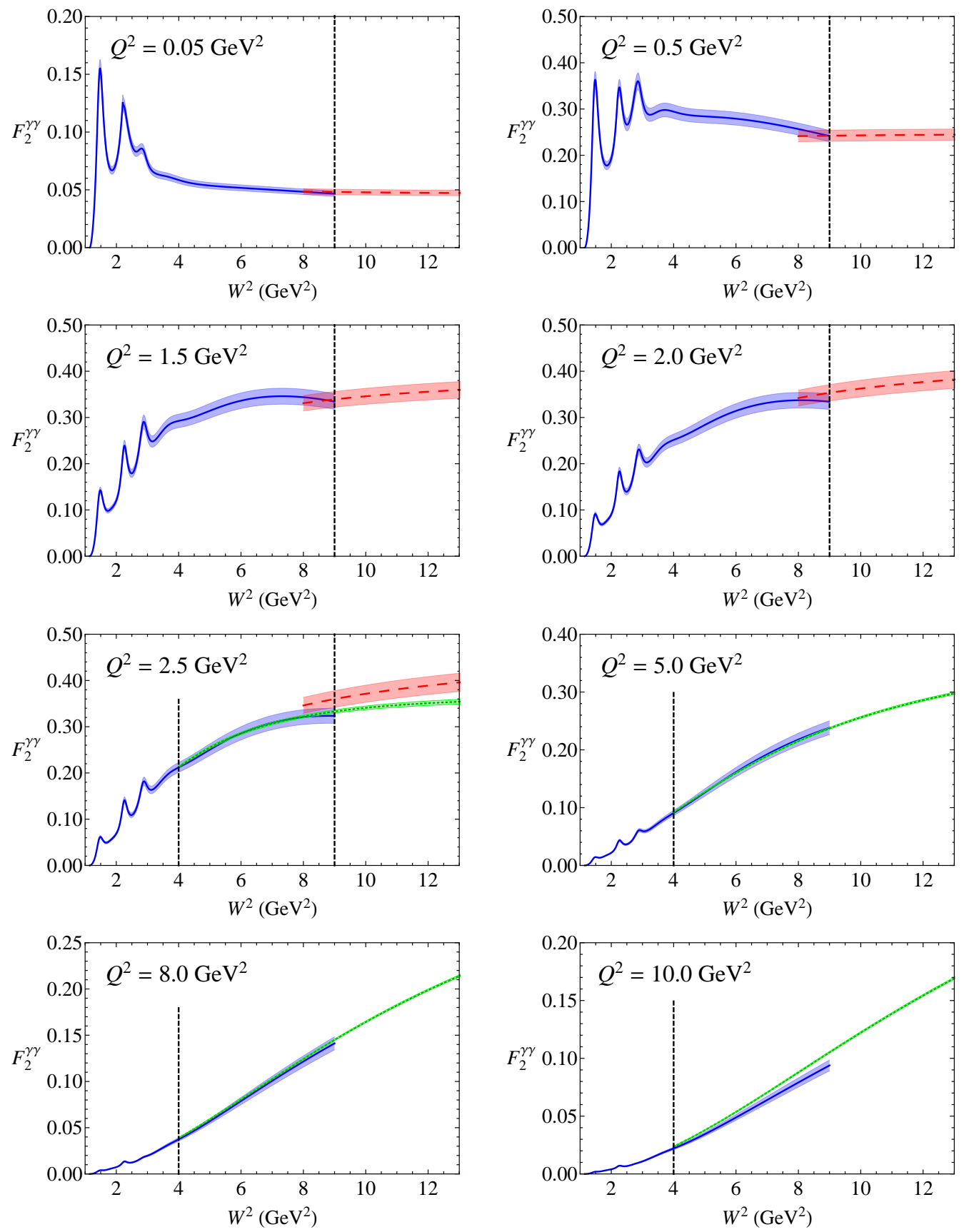

Figure 5.2: Proton $F_{2}^{\gamma \gamma}$ structure function versus $W^{2}$ at various fixed $Q^{2}$ values for the low- $W$ CB fit [121] (blue solid), the high- $W$ VMD+Regge [134] (red dashed) and ABM11 [141] (green dotted) parametrisations. The boundaries between the Regions I, II and III are indicated by the vertical lines at $W^{2}=4$ and $9 \mathrm{GeV}^{2}$. 

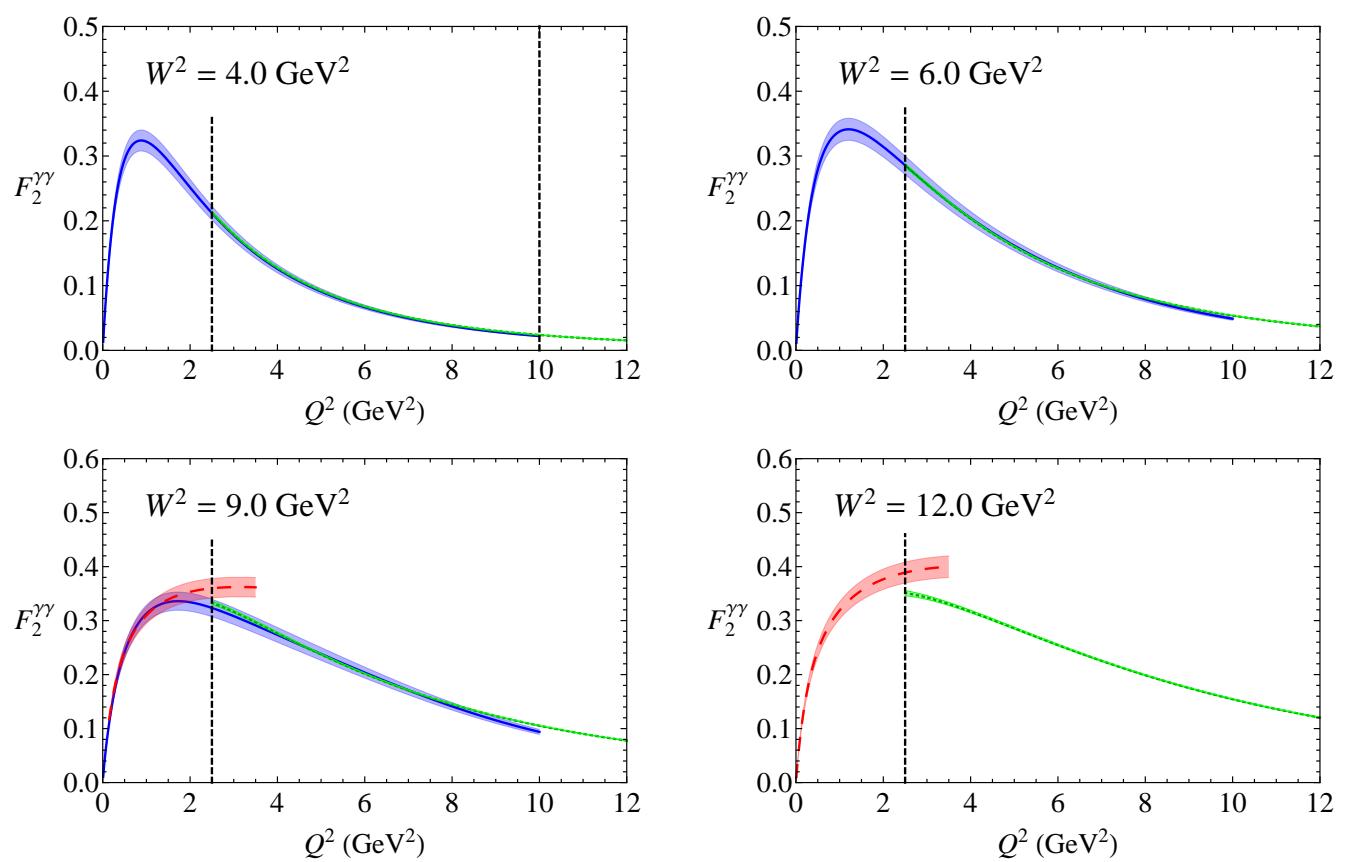

Figure 5.3: As in Fig. 5.2 but with the proton $F_{2}^{\gamma \gamma}$ structure functions held fixed at $W^{2}=4,6,9$ and $12 \mathrm{GeV}^{2}$ and plotted against $Q^{2}$.

in $Q^{2}$ and $W^{2}$ than shown here, the lack of resonance structure means that the parametrisation of $\mathrm{CB}$ more accurately exhibits the physics behind these functions. Of course, alternative PDF fits such as those used in Refs. [142, $143,144,145,120]$ could also be used instead. (An evaluation of $\square_{\gamma Z}$ using the structure functions from Ref. [120] yielded similar results.)

Just as it was important that the CB and VMD+Regge parametrisations matched at their boundaries, so is the case with the DIS structure functions. In the bottom half of Fig. 5.2 we plot the DIS $F_{2}^{\gamma \gamma}$ structure function along with the $\mathrm{CB}$ and VMD+Regge parametrisations. ${ }^{2}$ As before, the overlap between the different regions is very good.

In addition to plotting $F_{2}^{\gamma \gamma}$ as a function of $W^{2}$ - while holding $Q^{2}$ fixedit is helpful to see the transition between regions as $Q^{2}$ varies. Figure 5.3 illustrates this for several values of $W^{2}$ and shows that along this section of the $Q^{2}-W^{2}$ plane, the structure functions of Alekhin et al. and $\mathrm{CB}$ match exceptionally well. In the low $W^{2}$ region where all three are valid there is also good agreement. However, at higher $W^{2}\left(W^{2} \gtrsim 10 \mathrm{GeV}^{2}\right)$, the VMD+Regge model deviates from the DIS structure functions at the boundary between Regions II and III. Nevertheless since the contribution to the $\square_{\gamma Z}^{V}$ correction at these $W^{2}$ values is small, the effect is negligible.

\footnotetext{
${ }^{2}$ We may include the VMD+Regge structure function at $Q^{2}=2.5 \mathrm{GeV}^{2}$ since it is still valid at this momentum.
} 


\section{2 $\quad$ AJM $\gamma Z$ interference structure functions}

The next step in the procedure involves modifying the above model structure functions to obtain expressions for their interference analogues. In this section we begin by transforming the $\mathrm{CB}$ parametrisation, before following with the construction of the VMD+Regge model interference structure functions. Having laid out these details we discuss the PDFs used in the DIS region of the dispersion integral.

For the resonance part, $\sigma_{T, L}^{(\mathrm{res})}$, of Regions I and II, the total cross section [121],

$$
\sigma_{T, L}=\sigma_{T, L}^{(\mathrm{res})}+\sigma_{T, L}^{(\mathrm{bgd})}
$$

we use the ratio $\xi_{R}$ given by Eq. (4.28) and $y_{R}$ defined in terms of the nucleon transition amplitudes (as in Eq. (4.29)), to modify each of the seven resonances present in the fit. Although this method follows that of GHRM [110], the assignment of uncertainties to $\xi_{R}$ for each of these resonances differs significantly. In Ref. [110] the errors on the $y_{R}$ parameters are found by taking the extremal values of the helicity amplitudes $A_{R, \lambda}^{N}$. This assumes that the distribution is uniform which is in marked contrast to the more conventional Gaussian distribution. By adding the uncertainties linearly, GHRM overestimate the errors on $\xi_{R}$ and thus for the AJM model, we use the standard Gaussian approach, adding the errors in quadrature. Note, however, that when GHRM combine the overall errors from different segments for the final $\Re e \square_{\gamma Z}^{V}$ value, they add them in quadrature.

A comparison between the two methods for adding the total helicity amplitude errors is shown in Table 5.1. In this table we present the calculated $y_{R}$ for the proton, neutron and deuteron. (Only the errors added in quadrature are shown for the latter two.) Although it is just the proton's $y_{R}$ values which are needed in the $\square_{\gamma Z}^{V}$ calculation, for the evaluation of the PVDIS asymmetry, the deuteron's will also be required. While the PDG [3] assigns zero uncertainty to the (isospin- $\left.\frac{3}{2}\right) P_{33}(1232)$ and $F_{37}(1950)$ resonances' helicity amplitudes, in order to be conservative we follow GHRM [110] in placing a $10 \%$ error on the $P_{33}(1232)$ amplitude and a $100 \%$ error on the $F_{37}(1950)$ resonance.

In the course of this work the determinations of the helicity amplitudes in the PDG were updated, which allowed us to use these latest values in our numerical calculations. To compare with GHRM, however, we also computed $y_{R}$ and its uncertainties using the earlier 2010 PDG values [136]. Since the only resonances whose values differ across the two versions are the $D_{13}(1520)$ and $P_{11}(1440)$ resonances the overall effect is minimal. In order to be complete we include the earlier values in parentheses in Table 5.1 where the errors have again been added in quadrature.

For the background piece in Eq. (4.17) the electromagnetic cross sections are transformed to their $\gamma Z$ analogues via the ratio in Eq. (4.32) - as in Model II of Ref. [110]. This ratio is used for both the kinematic region where the $\mathrm{CB}$ background is valid, and the VMD+Regge region. The distinction 
Table 5.1: Electromagnetic to $\gamma Z$ resonance cross section transformation ratios $y_{R}$ from Eq. (4.29) for the proton, neutron and deuteron in the AJM model, compared with the proton ratio in the GHRM model [110]. The AJM model values in parentheses use helicity amplitudes from the earlier 2010 PDG [136], as utilized by GHRM. The errors labeled with the asterisks (*) are values corrected [146] from those in Ref. [110].

\begin{tabular}{cccc}
\hline \hline & $P_{33}(1232)$ & $P_{11}(1440)$ & $D_{13}(1520)$ \\
\hline$p(\mathrm{AJM})$ & $-1.0 \pm 0.1$ & $-0.67 \pm 0.17$ & $-0.84 \pm 0.17$ \\
& & $(-0.62 \pm 0.16)$ & $(-0.77 \pm 0.08)$ \\
$p(\mathrm{GHRM})$ & $-1.0 \pm 0.1$ & $-0.62_{-0.20}^{+0.19}$ & $-0.77_{-0.125}^{+0.122}(*)$ \\
\hline$n$ (AJM) & $-1.0 \pm 0.1$ & $-1.50 \pm 0.39$ & $-0.85 \pm 0.15$ \\
\hline$d$ (AJM) & $-1.0 \pm 0.1$ & $-0.92 \pm 0.27$ & $-0.85 \pm 0.14$ \\
\hline \hline
\end{tabular}

\begin{tabular}{cccc}
\hline \hline$S_{11}(1535)$ & $S_{11}(1665)$ & $F_{15}(1680)$ & $F_{37}(1950)$ \\
\hline$-0.51 \pm 0.35$ & $-0.28 \pm 0.41$ & $-0.27 \pm 0.08$ & $-1 \pm 1$ \\
& & & \\
$-0.51_{-0.71}^{+0.35}$ & $-0.28_{-0.69}^{+0.45}(*)$ & $-0.27_{-0.12}^{+0.10}$ & $-1 \pm 1$ \\
\hline$-1.96 \pm 1.32$ & $-3.53 \pm 5.06$ & $-2.50 \pm 1.01$ & $-1 \pm 1$ \\
\hline$-0.81 \pm 0.64$ & $-0.52 \pm 0.78$ & $-0.49 \pm 0.14$ & $-1 \pm 1$ \\
\hline \hline
\end{tabular}

between the AJM description and Models I and II lies in the fact that here, instead of assuming the $\kappa_{C}^{T, L}$ parameters to be the same for the $\gamma \gamma$ and $\gamma Z$ continuum pieces, we determine $\kappa_{C}^{T, L}$ by insisting that the $\gamma Z$ structure functions match at the borders between the different regions. As illustrated in Fig. 5.1, these boundaries occur at $Q^{2}=2.5 \mathrm{GeV}^{2}$ and $W^{2}=9 \mathrm{GeV}^{2}$. By making this assumption, we will see that strong constraints are placed on $\kappa_{C}^{T, L}$ and results in the uncertainties of the background contribution being much smaller than those determined previously.

To complete the AJM model we use the PDF structure functions of Alekhin et al. $[141,147]$ in the region where $Q^{2}>2.5 \mathrm{GeV}^{2}$ and $W^{2}>$ $4 \mathrm{GeV}^{2}$. At the parton level, the modification to the $\gamma Z$ case is much more straightforward and is implemented by replacing the quark electric charges, $e_{q}$ (present in the PDF expressions of the $F_{i}^{\gamma \gamma}$ ), by the weak vector charges, $g_{V}^{q}$ i.e. $e_{q}^{2} \rightarrow 2 e_{q} g_{V}^{q}$ as in Eq. (4.14). ${ }^{3}$ Since there is no data for the interference structure functions at low $Q^{2}$, the values for the higher-twist contributions to $F_{i}^{\gamma Z}$ were taken to be the same as those in $F_{i}^{\gamma \gamma}$ [147]. In order to account for this lack of empirical knowledge, we assign a $5 \%$ uncertainty to the $F_{i}^{\gamma Z}$ in the DIS region. Note, however, that because of the nature of the longitudinal structure function which is expressed as a difference between the $F_{2}^{\gamma Z}$ and $F_{1}^{\gamma Z}$

\footnotetext{
${ }^{3}$ The additional factor of 2 takes into account the definitions of the $g_{V}^{q}$ used here.
} 
structure functions, the $F_{L}^{\gamma Z}$ relative uncertainties will necessarily be larger.

\subsection{Phenomenological constraints}

In Section 4.4 we pointed out that the central value of $\Re e \square_{\gamma Z}^{V}$ quoted in Ref. [110] is given by averaging Models I and II. With regards to the uncertainty, the background error is taken from the model with the larger of the two errors - in this case Model II. Since the background uncertainty is almost four times larger than the resonances uncertainty and dominates GHRM's final error, any reduction here would significantly lower the total $\Re e \square_{\gamma Z}^{V}$ uncertainty value. In order to do so, we examine background errors in Ref. [110] more closely.

Gorchtein et al. transform their nonresonant background by multiplying the VMD+Regge background with a $\gamma \gamma / \gamma Z$ cross section ratio,

$$
\sigma_{T, L}^{\gamma Z(\mathrm{bgd})}=\left(\frac{\sigma_{T, L}^{\gamma Z}}{\sigma_{T, L}^{\gamma \gamma}}\right) \sigma_{T, L}^{\mathrm{VMD}},
$$

where $\sigma_{T, L}^{\mathrm{VMD}}$ has been previously defined in Eqs. (4.24) and (4.25). The ratio $\left(\sigma_{T, L}^{\gamma Z} / \sigma_{T, L}^{\gamma \gamma}\right)$ used to rescale the electromagnetic cross section was defined in Eq. (4.32), with the uncertainties for $\kappa_{V}(V=\omega$ and $\phi)$, estimated by comparing the ratios $R_{V}^{T, L}$ with the values obtained using HERA's data on vector meson electroproduction [148]. The difference between the two was taken as the uncertainty. Since, as shown in Fig. 13 of Ref. [110], there is good agreement between the data and the VMD ratios, these uncertainties are small.

The final contribution to the background error comes from the continuum parameters $\kappa_{C}^{T, L}$ in Eq. (4.32). Because the VMD model provides no method for estimating $\kappa_{C}^{T, L}$ as it did for the $\kappa_{V}^{T, L}$ values, GHRM equate the $\gamma Z$ and $\gamma \gamma$ parameters before assigning a $100 \%$ uncertainty to $\gamma Z$ continuum parameters. In Fig. 5.4 we compare the resulting $F_{2}^{\gamma Z}$ structure functions with those given by the ABM11 parton distribution functions [141]. It is clear that the uncertainty on GHRM's structure function is significantly overestimated when compared with the global fits. Indeed, in Fig. 5.4 we have only included the uncertainty which comes from the $\kappa_{C}^{T, L}$ values, should the full set of uncertainties be incorporated, this difference would be even greater. In the region where both GHRM's model and the PDF parametrisation are valid, one would not expect such a difference in magnitude between the two uncertainties. Additionally, the central curves for the model lie significantly above those given by Alekhin et al.

In spite of the fact that the VMD description offers no insight into the true values of $\kappa_{C}^{T, L}$, we will see how parton distribution functions may be used to constrain the continuum term. Furthermore, the recent PV data from the Jefferson Lab E08-011 electron-deuteron scattering experiment [56, 93] will be used to provide additional checks on these constraints. By the end of this 

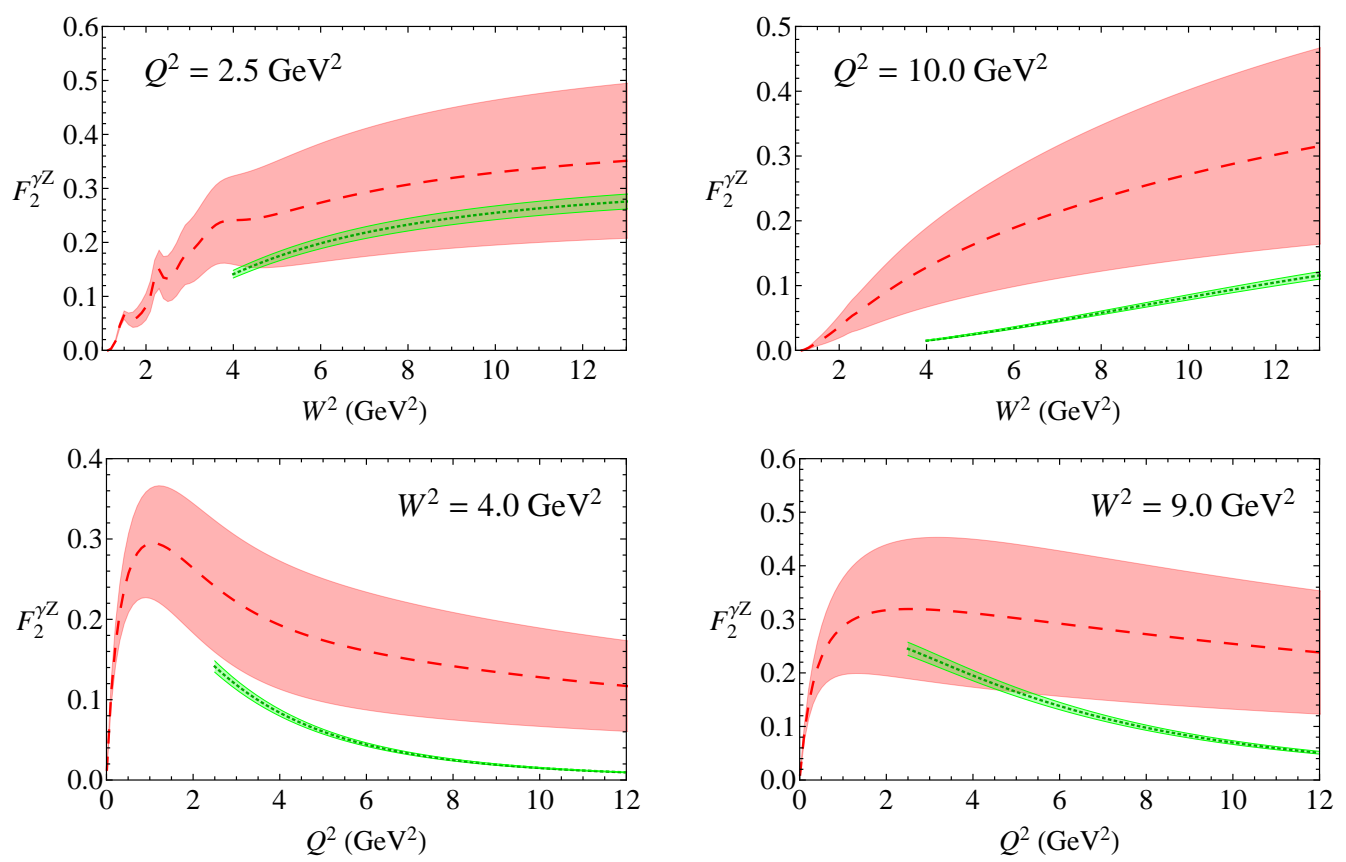

Figure 5.4: Comparison of the proton $F_{2}^{\gamma Z}$ structure function in the VMD+Regge model (Model II) of GHRM [110] (red dashed) with the ABM11 global parametrisation [141] (green dotted), for fixed $Q^{2}$ (top panels) and fixed $W^{2}$ (bottom panels). Note that the VMD+Regge model only includes uncertainties from the continuum part of the background, while the ABM11 parametrisation includes an overall $5 \%$ error.

analysis we will show that these constraints help to significantly reduce the uncertainty on the $F_{i}^{\gamma Z}$ structure functions.

\section{Constraints from PDFs}

At high $Q^{2}$ and $W^{2}$ DIS structure functions are well described by the leadingtwist PDFs. Additional corrections such as those coming from the target mass or higher twists can also be included to account for any remaining $1 / Q^{2}$ suppressed effects. However, as one moves into the low- $Q^{2}$, low- $W^{2}$ region nonperturbative physics begins to take over and the parton description breaks down. Nevertheless there remains a region in the $Q^{2}-W^{2}$ plane where the CB fit to data overlaps with global PDFs $[120,141,142,143,144,145]$. It is in this region where, by demanding that the CB-based parametrisation and the PDFs match, we may constrain the background contribution to the interference structure functions.

The AJM values for $\kappa_{C}^{T, L}$ are calculated by equating the cross section 
ratios $\sigma_{T, L}^{\gamma Z} / \sigma_{T, L}^{\gamma \gamma}$ in Eq. (4.32) with those given by the DIS structure functions,

$$
\frac{\sigma_{T}^{\gamma Z}}{\sigma_{T}^{\gamma \gamma}}=\left.\frac{F_{1}^{\gamma Z}}{F_{1}^{\gamma \gamma}}\right|_{\mathrm{DIS}}, \quad \frac{\sigma_{L}^{\gamma Z}}{\sigma_{L}^{\gamma \gamma}}=\left.\frac{F_{L}^{\gamma Z}}{F_{L}^{\gamma \gamma}}\right|_{\mathrm{DIS}},
$$

where we use the ABM11 parametrisation [141] for the PDF structure functions. (From Eqs. (4.10), (4.11) and the expression for the longitudinal structure function in terms of $F_{1}$ and $F_{2}$, it is relatively straightforward to show that left- and right-hand sides of the above equations are equivalent.) The uncertainties assigned to the PDFs are, 5\% uncertainty for the $F_{1}^{\gamma Z}$ and $40 \%$ uncertainty for $F_{L}^{\gamma Z}$, where as explained earlier, the larger error in the longitudinal structure function comes from that fact that it involves a difference between the $F_{1}^{\gamma Z}$ and $F_{2}^{\gamma Z}$. To be conservative these errors were chosen in spite of the fact that they are larger than those quoted by Alekhin et al. in Ref. [141].

Fitting for the continuum values required taking the minimum $\chi^{2}$ over a number of kinematic points in the $Q^{2}-W^{2}$ region where the descriptions overlapped. In order to check any dependence of the fitted $\kappa_{C}^{T, L}$ parameters on $Q^{2}$ and $W^{2}$, we held the momentum transfer fixed at $Q^{2}=2.5,6$ and $10 \mathrm{GeV}^{2}$ and considered several values of $W^{2}$ ranging from $4 \mathrm{GeV}^{2}$ to $13 \mathrm{GeV}^{2}$ for each $Q^{2}$ value. As can be seen from Fig. 5.5, although there is some dependence on the kinematics it is relatively small and for $\kappa_{C}^{T}$ decreases significantly with larger $Q^{2}$. The longitudinal piece on the other hand has, as might be expected, larger dependence on $W^{2}$ as $Q^{2}$ is increased.

The quoted values of the $\kappa_{C}^{T, L}$ parameters were determined by taking the average over the complete set of $Q^{2}$ and $W^{2}$ points, with the final value shown by the black horizontal line in Fig. 5.5. The error bars were calculated by combining uncertainties of the parton distributions with those coming from the $W^{2}$ dependence of $\kappa_{C}^{T, L}$. As the continuum parameters are correlated for each set of $Q^{2}$ values, a conventional $\chi^{2}$ fit would underestimate the errors. Instead we add in quadrature the values coming from the above mentioned uncertainties.

To estimate the $W^{2}$ dependence, we averaged the difference of the maximum and minimum $\kappa_{C}^{T}$ central values for the $Q^{2}$ set which showed the largest variation over $W^{2}$. For the transverse case this was $Q^{2}=2.5 \mathrm{GeV}^{2}$, while for $\kappa_{C}^{L}$ the largest variation occurred at $Q^{2}=10 \mathrm{GeV}^{2}$. The second set of errors comes from the PDFs and is given by the 'data' point which had the largest uncertainties. For both $\kappa_{C}^{T}$ and $\kappa_{C}^{L}$ this occurred at $Q^{2}=2.5 \mathrm{GeV}^{2}$.

Using this method for constraining the continuum parameters we found,

$$
\kappa_{C}^{T}=0.65 \pm 0.14, \quad \kappa_{C}^{L}=-1.3 \pm 1.7 .
$$

Comparing with those from Gorchtein et al., our uncertainty on $\kappa_{C}^{T}$ is approximately 5 times smaller. Interestingly, the $\kappa_{C}^{L}$ parameter is larger than GHRM's by $\sim 160 \%$. However, because the longitudinal part of the structure function only contributes a small amount to the final $\Re e \square_{\gamma Z}^{V}$ correction, this large error does not have an adverse effect on the final $\Re e \square_{\gamma Z}^{V}$ uncertainty. 

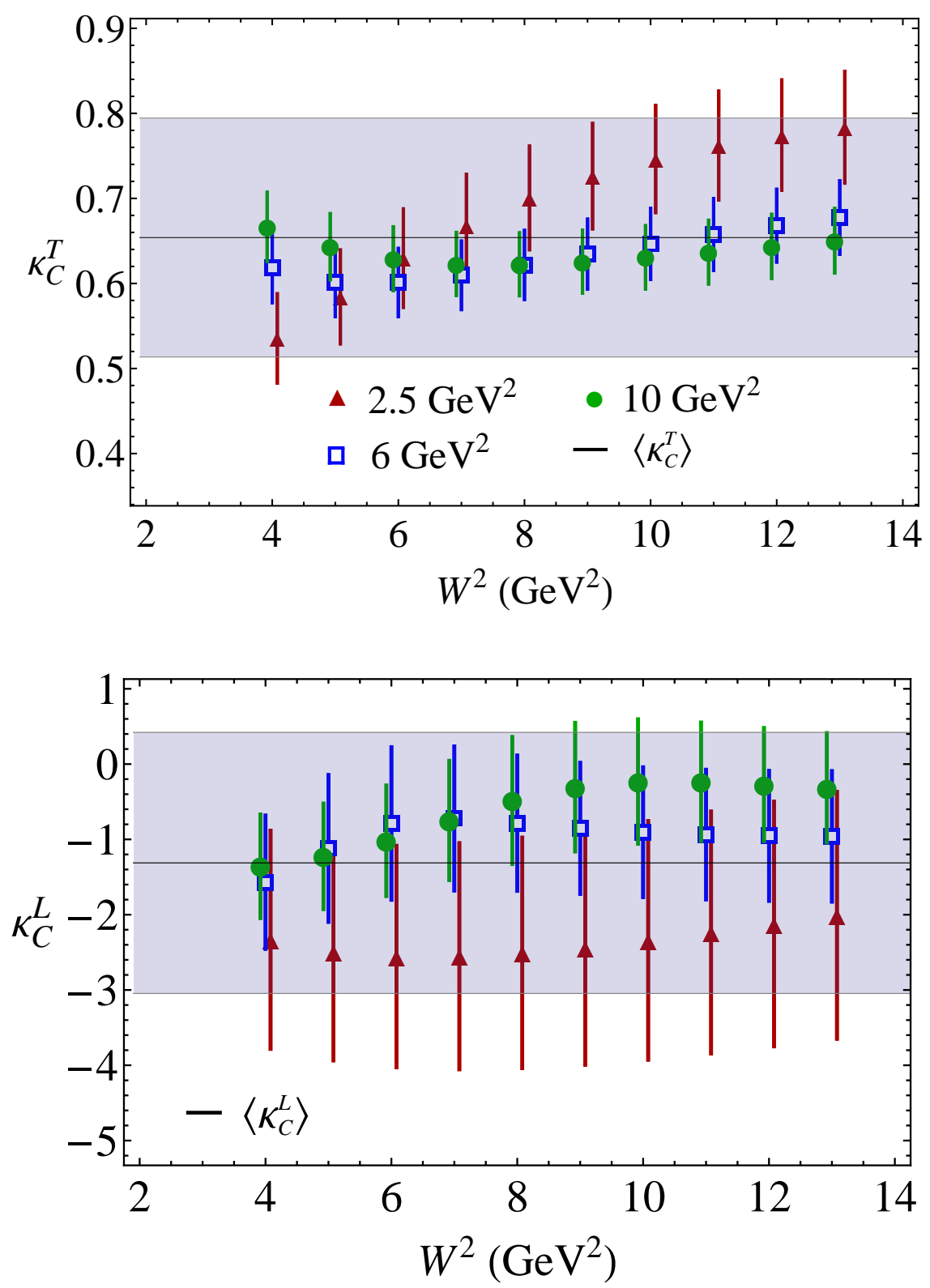

Figure 5.5: Continuum parameters $\kappa_{C}^{T}$ (top) and $\kappa_{C}^{L}$ (bottom) fitted to the DIS data, parametrised by the ABM11 global QCD fit [141], as a function of $W^{2}$ for fixed $Q^{2}=2.5 \mathrm{GeV}^{2}$ (red triangles), $6 \mathrm{GeV}^{2}$ (blue squares), and $10 \mathrm{GeV}^{2}$ (green circles). The average values $\left\langle\kappa_{C}^{T, L}\right\rangle$ are indicated by the solid lines, with the shaded band giving their uncertainty. Note that some of the points have been slightly offset for clarity. 

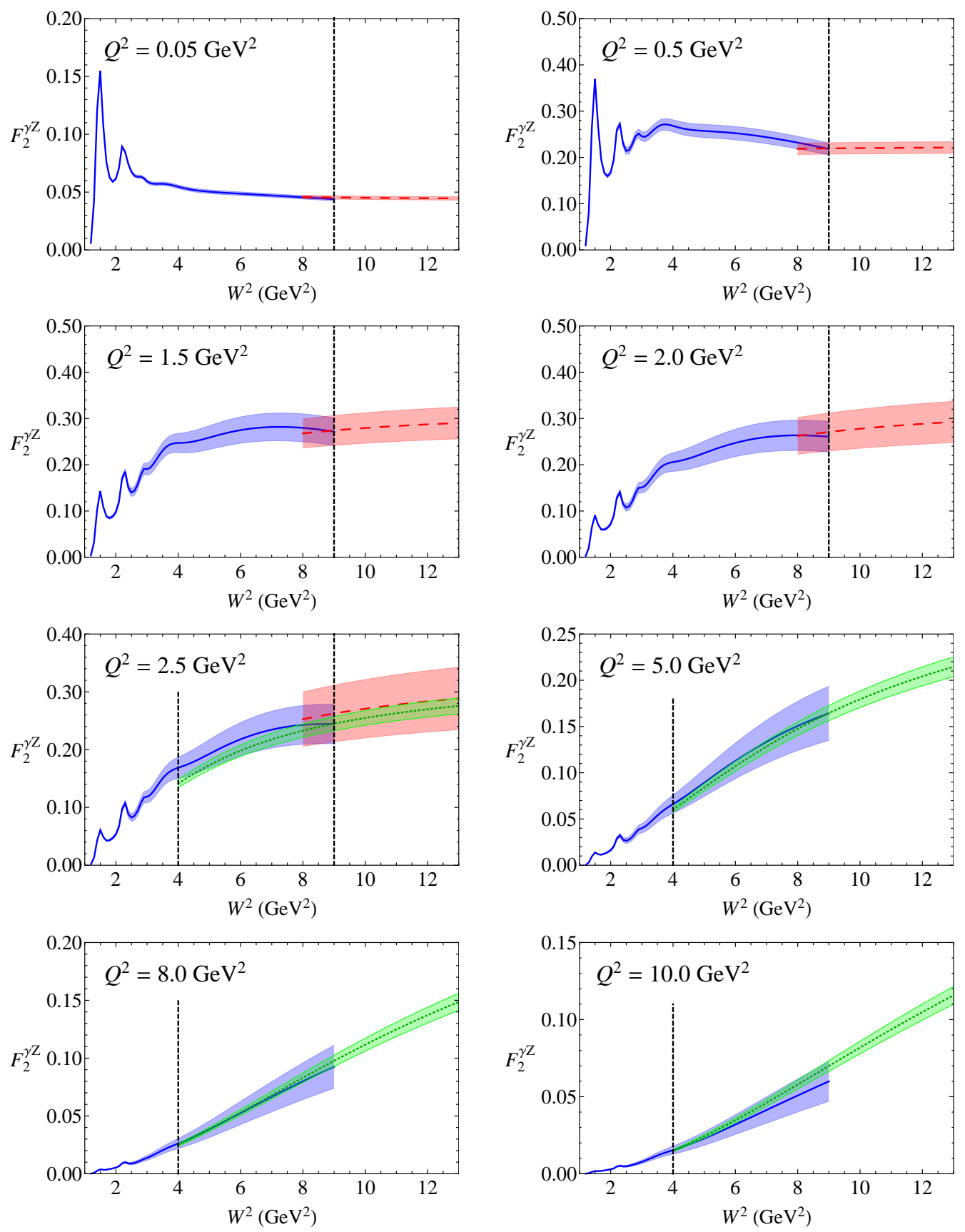

Figure 5.6: Proton $F_{2}^{\gamma Z}$ structure function versus $W^{2}$ at various fixed $Q^{2}$ values for the low- $W$ CB fit [121] (blue solid), the high- $W$ VMD+Regge [134] (red dashed) and ABM11 [141] (green dotted) parametrisations. The boundaries between the Regions I, II and III are indicated by the vertical lines at $W^{2}=4$ and $9 \mathrm{GeV}^{2}$. 

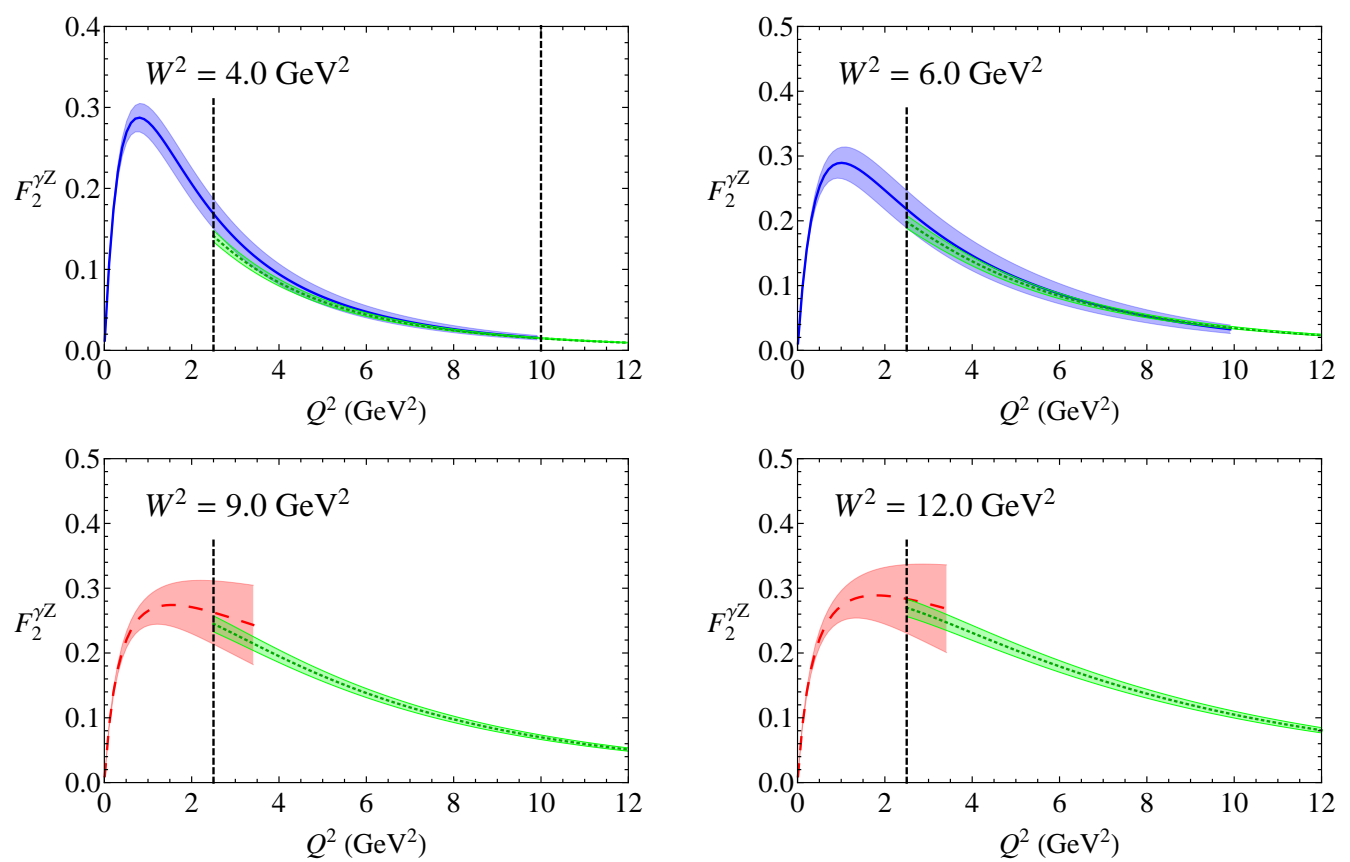

Figure 5.7: Proton $F_{2}^{\gamma Z}$ structure function versus $Q^{2}$ at fixed $W^{2}=4,6,9$ and $12 \mathrm{GeV}^{2}$ for the CB fit [121] (blue solid), the ABM11 PDF parametrisation [141] (green dotted), and the VMD+Regge model [134] (red dashed), with the boundaries between Regions I, II and III indicated by the vertical lines at fixed $Q^{2}$.

In Fig. 5.6 we plot the $F_{2}^{\gamma Z}$ structure function which results from constraining the $\kappa_{C}^{T}$ and $\kappa_{C}^{L}$ for $Q^{2}$ values ranging from $Q^{2}=0.05 \mathrm{GeV}^{2}$ to $10 \mathrm{GeV}^{2}$. As can be seen, the descriptions for the three regions agree remarkably well over the entire $Q^{2}$ range. Not only is there good overlap of the uncertainties, but the central values themselves show excellent matching at the boundaries. As in Fig. 5.4, the uncertainties shown here are only those which stem from the continuum parameters. This is so that we may compare directly with those errors estimated by GHRM. It is evident from Figs. 5.4 and 5.6 that the constraints placed on the continuum parameters have significantly reduced the uncertainty on the resulting $\gamma Z$ structure functions. This is also seen in Fig. 5.7 where we hold $W^{2}$ fixed and plot the structure functions as a function of $Q^{2}$.

For the total background uncertainty the errors coming from the vector meson ratios, $R_{\omega}^{T, L}$ and $R_{\phi}^{T, L}$, in Eq. (4.32) must also be included. As in Ref. [110] we determine the uncertainties for these values by taking the difference between the HERA experimental data [148] and the VMD model prediction. It is also assumed that the transverse and longitudinal ratios are equal, i.e. $R_{\omega}^{T}=R_{\omega}^{L}$ and $R_{\phi}^{T}=R_{\phi}^{L}$. By adding these uncertainties in quadrature with those coming from the continuum parameters and the resonances, we obtain the total error for the $\gamma Z$ structure functions.

The difference between the total uncertainties of the AJM model and 
GHRM's Model II may be further compared by evaluating the inelastic parityviolating asymmetry. Since there is data, albeit a small amount, on this phenomenon for both the proton and deuteron it is important to test both these models with the available experimental values. The asymmetry may be written as,

$$
A_{\mathrm{PV}}^{e}\left(\frac{G_{F} Q^{2}}{2 \sqrt{2} \pi \alpha}\right) \frac{x y^{2} F_{1}^{\gamma Z}+\left(1-y-\frac{x^{2} y^{2} M^{2}}{Q^{2}}\right) F_{2}^{\gamma Z}+\frac{g_{V}^{e}}{g_{A}^{e}}\left(y-\frac{1}{2} y^{2}\right) x F_{3}^{\gamma Z}}{x y^{2} F_{1}^{\gamma \gamma}+\left(1-y-\frac{x^{2} y^{2} M^{2}}{Q^{2}}\right) F_{2}^{\gamma \gamma}},
$$

where $F_{3}^{\gamma Z}$ is the axial-vector structure function.

As before, the cross section for the $F_{3}^{\gamma Z}$ is separated into a background part and a resonance part. For the resonances we use the parametrisation of Lalakulich et al. [149, 150, 151] who give expressions for the axial-vector transition form factors, while background part of the cross section follows CR's example [123] and uses a scaled version of the electromagnetic cross section. This is done by taking the average of the $x \rightarrow 0$ limit, where $F_{3}^{\gamma Z}=0$ and the quark model limit, where $F_{3}^{\gamma Z}=10 / 3 F_{1}^{\gamma \gamma}$ thus giving, $F_{3}^{\gamma Z}=5 / 3 F_{1}^{\gamma \gamma}$ [123]. In the case of the deuteron, the rescaling factor becomes, $F_{3}^{\gamma Z}=9 / 5 F_{1}^{\gamma \gamma}$. Since the contribution to the overall asymmetry from the axial-vector structure function is small $(\sim 10 \%)$ the $100 \%$ uncertainty assigned to this structure function will not have an overly large effect on the total asymmetry.

In Fig. 5.8 we compare the AJM and GHRM models' determination of the inelastic asymmetry with the value measured by the G0 experiment at JLab [91]. With a beam energy of $E=0.69 \mathrm{GeV}$ and momentum transfer $Q^{2}=0.34 \mathrm{GeV}^{2}$, this data point sits in the kinematic area of the $\Delta$ resonance region. Although the central values show good agreement with the data, the large uncertainty prevents any additional constraints from being placed on the $\gamma Z$ structure functions. As expected, the uncertainties on the constrained AJM model predictions are smaller than those predicted using Model II of Ref. [110]. This is especially so in the higher $W$ region. The asymmetry in the errors follows from the fact that we use the upper and lower values of the $F_{i}^{\gamma Z}$ structure functions in Eq. (5.4) in order to compute the $A_{\mathrm{PV}}$ uncertainty.

At larger $Q^{2}$ where JLab experiments more commonly explore, Fig. 5.9 shows that for the kinematics, $E=6 \mathrm{GeV}$ and $Q^{2}=2.5 \mathrm{GeV}^{2}$, the difference between the uncertainties of the two models is even more pronounced. In this kinematic region the errors computed using the GHRM model are $\sim 4$ times greater than those calculated using the constrained AJM structure functions. Further comparison between the PDF [141] asymmetry is given by the dotted green line - shown only as far as $W=2 \mathrm{GeV}$, since below this region the perturbation theory is no longer a good description of the physics.

While the AJM model's error is certainly larger than that of the PDF parametrisation, it remains of a compatible magnitude. The GHRM model on 

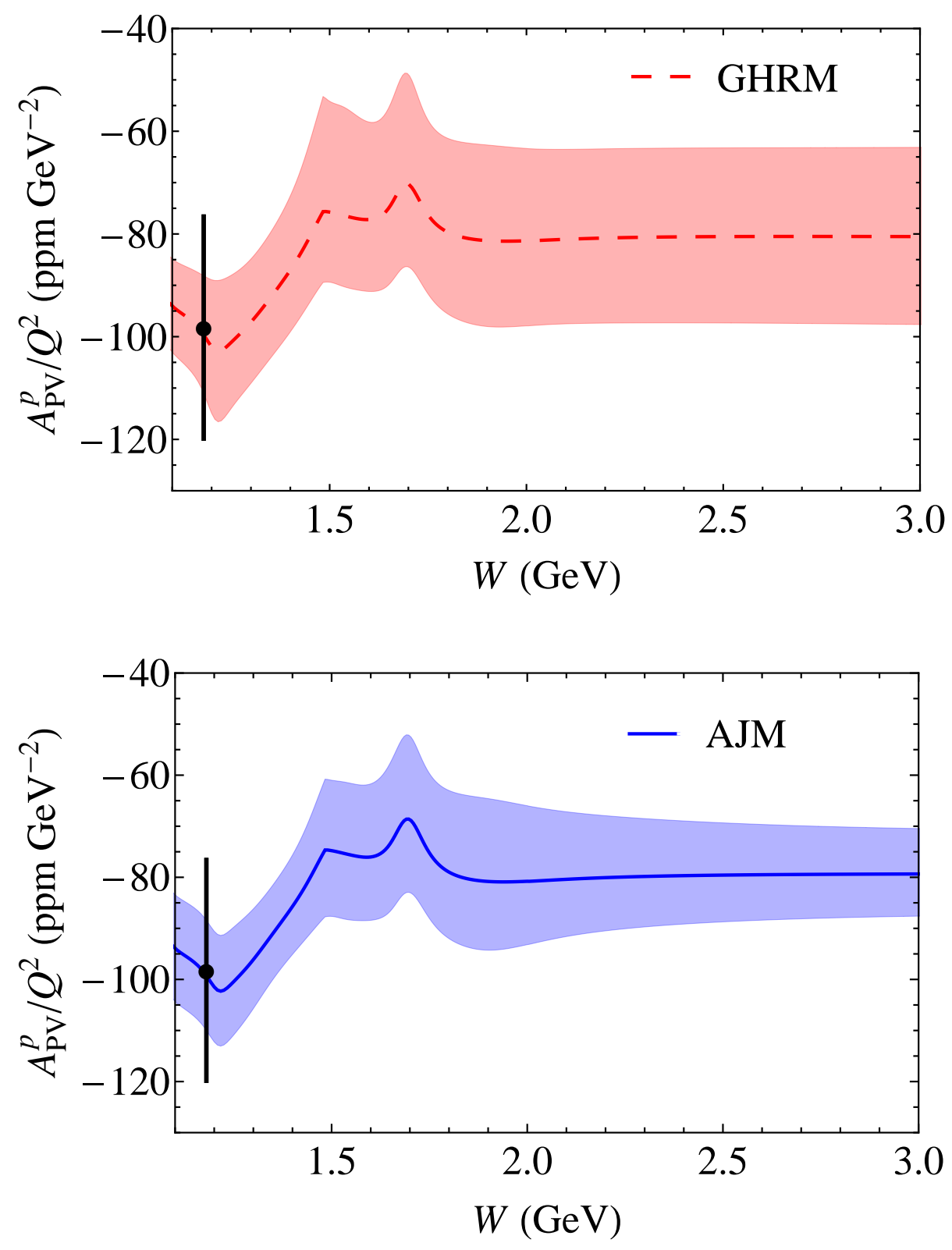

Figure 5.8: Proton parity-violating inelastic asymmetry $A_{\mathrm{PV}} / Q^{2}$, measured in parts per million (ppm) $\mathrm{GeV}^{-2}$, as a function of $W$, at fixed incident energy $E=0.69 \mathrm{GeV}$ and $Q^{2}=0.34 \mathrm{GeV}^{2}$, for the GHRM Model II [110] (top) and the AJM model (bottom). The data point at $W=1.18 \mathrm{GeV}$ (black circle) is from the Jefferson Lab G0 experiment [91]. 

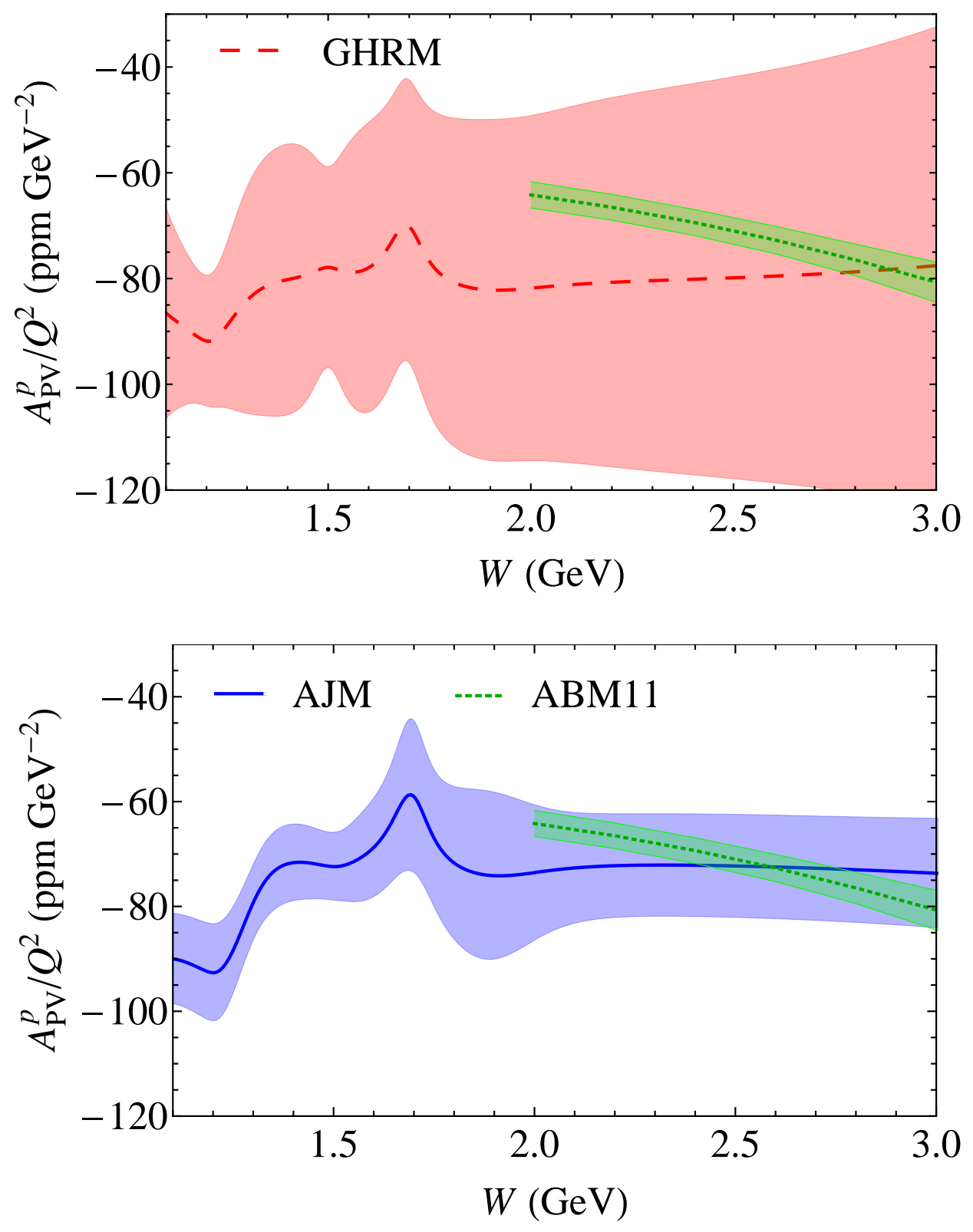

Figure 5.9: Proton parity-violating inelastic asymmetry $A_{\mathrm{PV}} / Q^{2}$ as a function of $W$, at fixed incident energy $E=6 \mathrm{GeV}$ and $Q^{2}=2.5 \mathrm{GeV}^{2}$, for the GHRM Model II [110] (top) and the AJM model (bottom). The asymmetry computed directly from PDFs [141] is represented by the green band. 
the other hand clearly overestimates the asymmetries errors in the kinematic region where the DIS structure functions are also valid. Although the PDF parametrisations are not of themselves accurate descriptions of the structure functions in the resonance region, we emphasise that the constraints which are placed by the boundary matching conditions mean that the errors on the interference structure functions in the low- $Q^{2}$, low- $W^{2}$ region must also be significantly constrained. In the next section we test the AJM model further by comparing with recent PVDIS data from electron-deuteron scattering.

\section{Deuteron asymmetry}

In 2013, the E08-011 experiment released results for PV electron-deuteron inelastic scattering in the resonance region [56]. This was followed by DIS asymmetries published earlier this year [93]. While the DIS data was unavailable at the time, we were able to use the resonance data to independently check the accuracy of the $\gamma Z$ structure functions constructed using our procedure. Given the large dependence of $\Re e \square_{\gamma Z}^{V}$ on the resonance region, the investigation of the resonance structure functions is particularly important. Furthermore, the AJM model could still be used to predict the DIS asymmetries of which the numerical values are shown in Table 5.2. In Chapter 6 we compare these predictions with the experimental results.

The asymmetry was measured at $W=1.26,1.59,1.86$ and $1.98 \mathrm{GeV}$, with the $Q^{2}$ values extending from as low as $0.76 \mathrm{GeV}^{2}$ and up to as much as $1.47 \mathrm{GeV}^{2}$. These data points are compared with the AJM model determinations in Fig. 5.10. (Dividing the deuteron PV asymmetry, $A_{\mathrm{PV}}^{d}$, by $Q^{2}$ allows the various experimental values to be displayed on the same plot.) As before, the input deuteron structure functions have been constrained by using the parton description [141], where $F_{i}^{d}$ is simply,

$$
F_{i}^{d}=F_{i}^{p}+F_{i}^{n} ; \quad i=1,2, L
$$

and this is true for both the electromagnetic and interference structure functions. For the deuteron, the constraints from PDFs give $\kappa_{C}^{T}(d)=0.79 \pm 0.05$ for the continuum parameter of the transverse cross section. The resulting asymmetry is for the most part, in excellent in agreement with the experimental data [56], although at $Q^{2}=0.95 \mathrm{GeV}^{2}$ where the $\Delta$ resonance dominates there is a small difference.

It is difficult to understand theoretically the variation between the model prediction and the E08-011 $\Delta$ resonance data point since the theory value depends only on isospin symmetry and the assumption that vector current is conserved. Perhaps the discrepancy is a result of the nonresonant background having a stronger isospin dependence [152] than previously estimated. However, the difference is still $\lesssim 2 \sigma$. Furthermore, from Fig. 5.8 it is clear that the models agree well with the $\Delta$ resonance G0 data [91] point even though the errors are significantly larger.

For the longitudinal continuum parameter, the matching with the global PDF structure functions of Ref. [141] give $\kappa_{C}^{L}(d)=0.2 \pm 3.4$. The propagation 

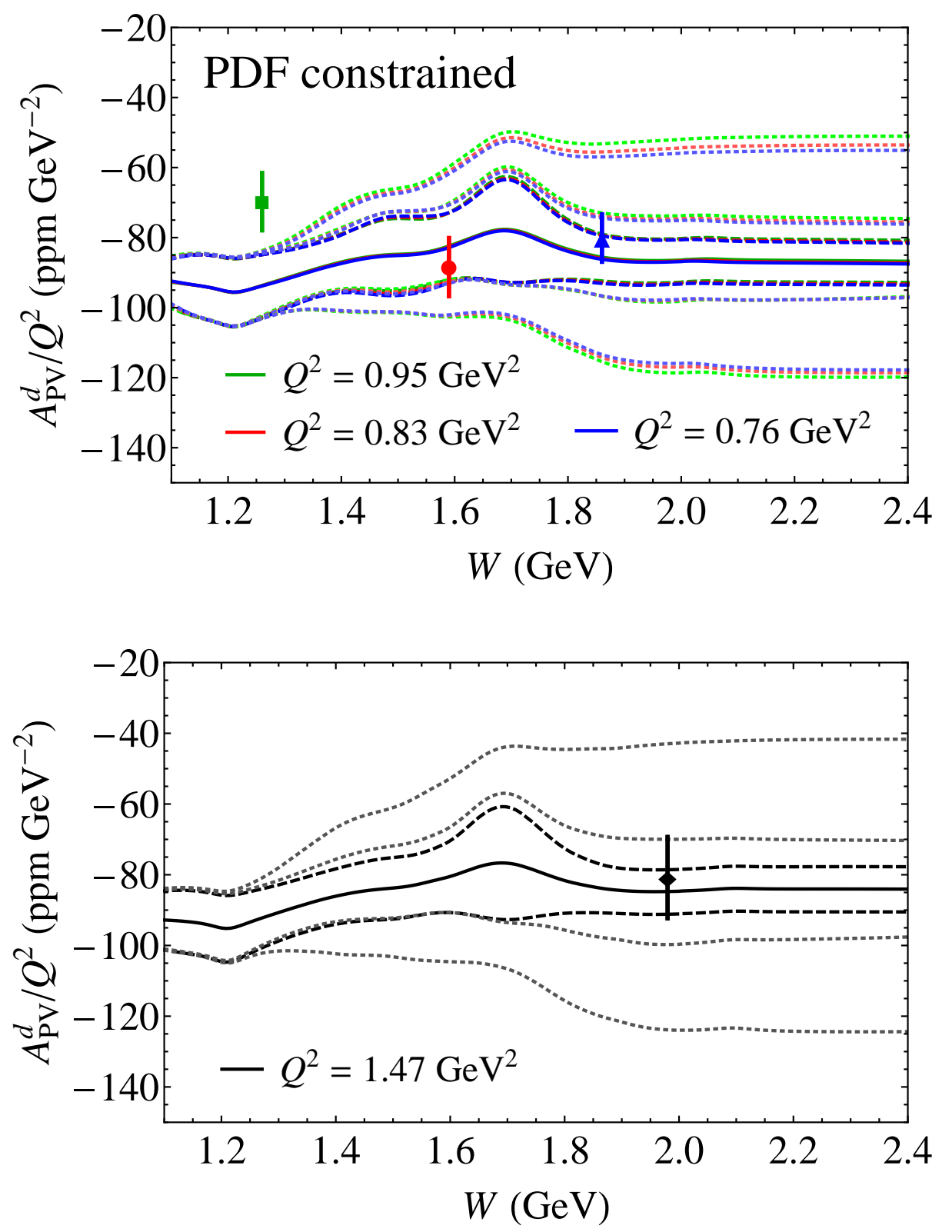

Figure 5.10: Deuteron parity-violating asymmetry $A_{\mathrm{PV}}^{d} / Q^{2}$ as a function of $W$ for incident electron energy $E=4.9 \mathrm{GeV}$ (top) and $E=6.1 \mathrm{GeV}$ (bottom). The data points from the Jefferson Lab E08-011 experiment [56] at $W=1.26$ (green square), 1.59 (red circle), 1.86 (blue triangle) and $1.98 \mathrm{GeV}$ (black diamond) correspond to average values of $Q^{2}=0.95,0.83,0.76$ and $1.47 \mathrm{GeV}^{2}$, respectively. The AJM model uncertainties (inner dashed band) are constrained by matching the continuum parameters $\kappa_{C}^{T, L}(d)$ to the DIS region $\gamma Z$ structure functions [141], and are compared with those computed with errors on $\kappa_{C}^{T, L}(d)$ of $100 \%$ (outer dotted bands) and $25 \%$ (inner dotted bands). 

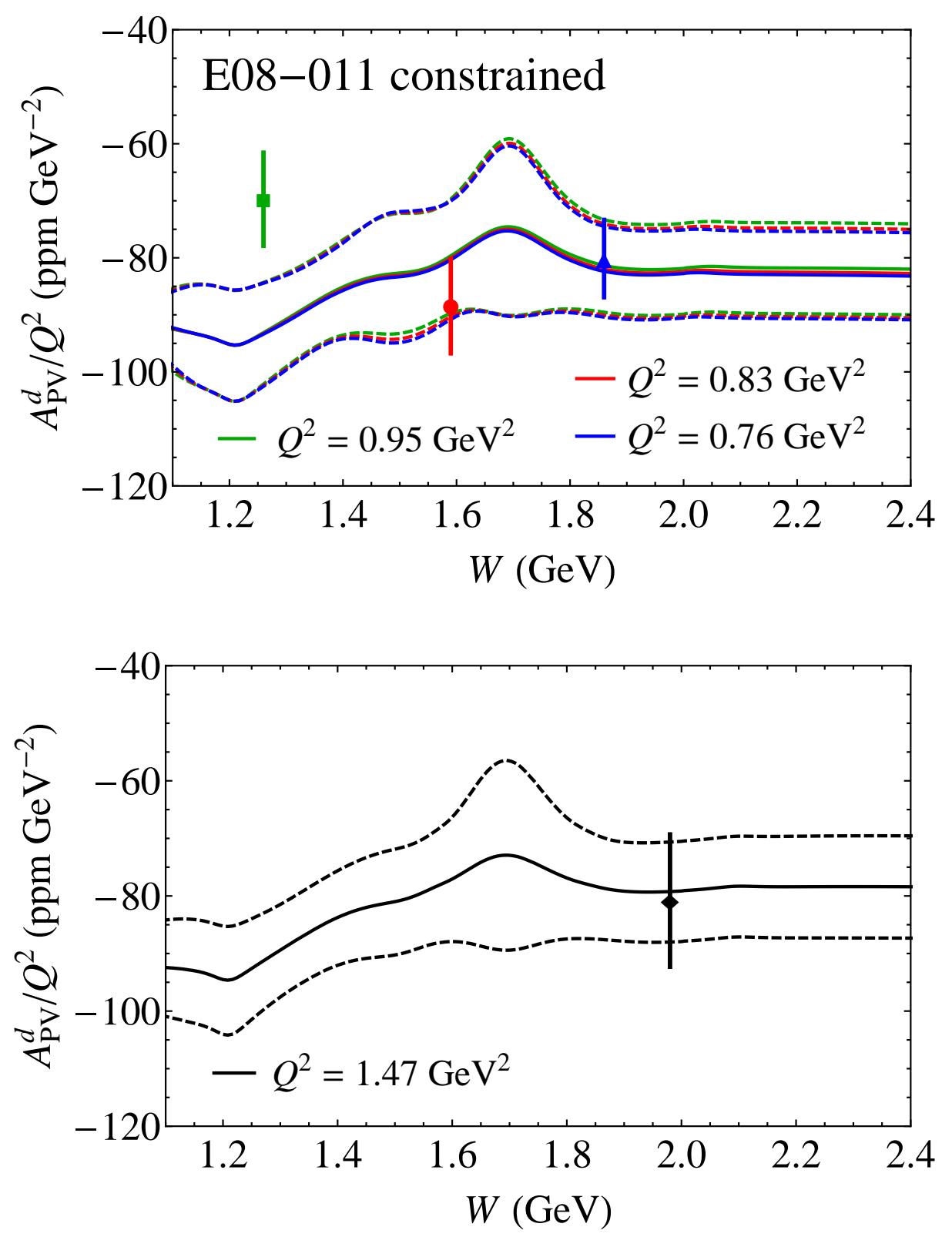

Figure 5.11: As in Fig. 5.10, but with the AJM model asymmetries (solid) and their uncertainties (dashed) constrained by the E08-011 data [56]. Note the different scale on the $y$-axis to that in Fig. 5.10. 
Table 5.2: Parity-violating deuteron asymmetries in the AJM model at the kinematics of the E08-011 experiment [56]. The asymmetries are computed with the continuum parameters $\kappa_{C}^{T, L}(d)$ constrained by the E08-011 data, or by matching to the DIS region described in terms of PDFs. Note that the points marked with asterisks $\left(^{*}\right)$ are predictions.

\begin{tabular}{ccccc}
\hline \hline & & & \multicolumn{2}{c}{$A_{\mathrm{PV}} / Q^{2}\left(\mathrm{ppm} \mathrm{GeV}^{-2}\right)$} \\
$E(\mathrm{GeV})$ & $W(\mathrm{GeV})$ & $Q^{2}\left(\mathrm{GeV}^{2}\right)$ & PDF constrained & E08-011 constrained \\
\hline 4.9 & 1.26 & 0.95 & $-93.7_{-9.0}^{+8.8}$ & $-93.1_{-9.0}^{+8.8}$ \\
4.9 & 1.59 & 0.83 & $-82.7_{-9.9}^{+9.7}$ & $-80.1_{-10.3}^{+10.1}$ \\
4.9 & 1.86 & 0.76 & $-86.2_{-6.9}^{+6.7}$ & $-82.4_{-8.0}^{+7.9}$ \\
6.1 & 1.98 & 1.47 & $-84.7_{-6.4}^{+6.2}$ & $-79.2_{-8.8}^{+8.6}$ \\
\hline 6.1 & 2.03 & 1.28 & $-84.9_{-6.4}^{+6.2}(*)$ & $-79.7_{-8.6}^{+8.4}(*)$ \\
6.1 & 2.07 & 1.09 & $-85.2_{-6.4}^{+6.2}(*)$ & $-80.3_{-8.3}^{+8.2}(*)$ \\
6.1 & 2.33 & 1.90 & $-82.7_{-6.5}^{+6.3}(*)$ & $-76.5_{-9.3}^{+9.3}(*)$ \\
\hline \hline
\end{tabular}

of this error into the total $A_{\mathrm{PV}}^{d}$ uncertainty is problematic as a result of the nature of the CB parametrisation [121], we may, however, use the uncertainties of the proton $\kappa_{C}^{T, L}$ values to take into account the errors from the longitudinal continuum parameter instead. The uncertainties for both $\kappa_{C}^{T}$ and $\kappa_{C}^{L}$ have been included in Fig. 5.10. For comparison, we also include in the plot an estimate of the error using a $100 \%$ uncertainty for the continuum parameters as in Ref. [110]. By following GHRM's example, the uncertainty for the PV asymmetry at $W \gtrsim 1.8 \mathrm{GeV}$ is approximately 6 times larger than those from the AJM prediction. Even with a $25 \%$ error, the total uncertainty on $A_{\mathrm{PV}}^{d}$ is larger than the AJM model's, although they are more compatible than GHRM's.

In the same way that the continuum parameters were constrained using the PDFs, as a check, we constrain the $\kappa_{C}^{T}(d)$ by fitting to the E08-011 data points instead. Such a fit results in $\kappa_{C}^{T}(d)=0.69 \pm 0.13$, which agrees well with the PDF constraints within errors. (Alternatively, one may omit the discrepant $\Delta$ point from the fit and obtain a slightly greater value, $\kappa_{C}^{T}(d)=0.72 \pm 0.13$.) Since the CB parametrisation provides only the $F_{1}^{\gamma \gamma}$ deuteron structure function explicitly - the longitudinal $F_{L}^{\gamma \gamma}$ is obtained by the ratio $R=\sigma_{L}^{\gamma \gamma} / \sigma_{T}^{\gamma \gamma}$ which is assumed to be the same for the deuteron as the proton-it is not possible to use the experimental data points to directly constrain the longitudinal continuum parameter as previously. Nevertheless, the longitudinal structure function errors may still be propagated through to the asymmetry by including the uncertainties of the $\kappa_{C}^{T, L}$ values for the proton in the analogous $\sigma_{L}^{\gamma Z} / \sigma_{T}^{\gamma Z}$ ratio. Ideally, a parametrisation of the deuteron $F_{L}^{\gamma \gamma}$ would be constructed explicitly which would allow for a more accurate determination of $\kappa_{C}^{L}(d)$ and straightforward propagation of errors.

Once more the predicted asymmetries are in good agreement with the $e-d$ scattering data. Comparing Fig. 5.11 with 5.10 it is also clear that the two methods give very similar predictions, with the data constrained central 
$A_{\mathrm{PV}}^{d}$ values only marginally higher than those constrained by PDFs. Furthermore, the uncertainties which result remain three to four times smaller for $W \gtrsim 1.8 \mathrm{GeV}$ than those estimated using the $100 \%$ uncertainty. Even the $25 \%$ errors give final asymmetry uncertainties which are larger than those from data constraints. The agreement between the two methods of predictions and the data points gives us confidence in the reliability of our method for constraining the proton $\gamma Z$ structure functions in the low- $Q^{2}$, low- $W^{2}$ region which dominates the $\Re e \square_{\gamma Z}^{V}$ correction.

To complete this section, in Table 5.2 we show the determination of the deuteron asymmetries and their uncertainties calculated using the constraints coming from the PDFs and those from the E08-011 experiment. The AJM model's prediction (identified by asterisks) for the DIS asymmetries are also included in this table. Now that we have a construction of the proton $\gamma Z$ structure functions, and successfully shown the reliability of this model, the $\square_{\gamma Z}^{V}$ correction may be calculated. In the following chapter we show the energy dependence of this contribution and determine the size of its uncertainty for the $Q_{\text {weak }}$ and MOLLER experiments. 



\section{The $\square_{\gamma Z}^{V}$ correction}

Having constructed the AJM model using constraints from parton distributions and having shown the reliability of this model by testing it with empirical data from the PV electron-deuteron experiment, we are now in the position to calculate the $\square_{\gamma Z}^{V}$ correction. Furthermore, since we have developed a good understanding of where the uncertainties in the AJM model come from and why they differ from Model II of Gorchtein et al. [110], it will be relatively straightforward to understand any differences found in the estimated uncertainty on the $\gamma Z$ box. Given the recent debate surrounding the $\square_{\gamma Z}^{V}$ correction and the size of that uncertainty, this is particularly important.

In this chapter, the energy dependence of $\Re e \square_{\gamma Z}^{V}$ and its associated error are examined in some detail. While the $\Re e \square_{\gamma Z}^{V}$ correction needs to be determined for both $Q_{\text {weak }}$ and MOLLER, the variation in the experimental kinematics of the two means that different aspects of the calculation need to be emphasised. These calculations are therefore dealt with in separate sections. In Section 6.1 we examine the $\Re e \square_{\gamma Z}^{V}$ correction to $Q_{\text {weak }}$, while Section 6.3 contains the MOLLER analysis. A comparison with earlier work is possible for the $Q_{\text {weak }}$ evaluation, however, for the larger energies, $\Re e \square_{\gamma Z}^{V}$ has not yet been calculated and thus our work represents the first determination of this important background correction to the MOLLER experiment at $\sim 11 \mathrm{GeV}$. In between these two sections, the parity-violating DIS asymmetries which were predicted in Table 5.2 of Chapter 5 will be revisited and compared with the results from Ref. [93]. We will also predict the proton inelastic asymmetry for a possible $Q_{\text {weak }}$ measurement [153] in this section.

Because of the nature of the break up of the various regions' contributions to $\Re e \square_{\gamma Z}^{V}$ at MOLLER energies, additional checks on the model dependence will be required. As we will see, Region II dominates the MOLLER $\Re e \square_{\gamma Z}^{V}$ contribution and by constructing a number of other models, we investigate the possible model dependence of the Regge contribution and how this might affect the final uncertainty. Another possible source of error lies in the kinematic dependence of the continuum parameters; this issue is discussed for both $Q_{\text {weak }}$ and MOLLER. Note that since $\kappa_{C}^{L}$ plays only a small role in the final uncertainty we may concentrate simply on the transverse case. Finally, we examine what effects the 'hard' boundaries have on the determina- 
Table 6.1: Contributions to $\Re e \square_{\gamma Z}^{V}$ from various regions in $Q^{2}$ and $W^{2}$ in the AJM model (see Fig. 5.1) at the $Q_{\text {weak }}$ energy $E=1.165 \mathrm{GeV}$.

\begin{tabular}{lc}
\hline \hline Region & $\Re e \square_{\gamma Z}^{V}\left(\times 10^{-3}\right)$ \\
\hline I (res) & $2.18 \pm 0.29$ \\
I (bgd) & $2.46 \pm 0.20$ \\
I (total) & $4.64 \pm 0.35$ \\
II & $0.59 \pm 0.05$ \\
III & $0.35 \pm 0.02$ \\
\hline Total & $5.57 \pm 0.36$ \\
\hline \hline
\end{tabular}

tion of the $\gamma Z$ box. This analysis is necessary for both PV electron scattering experiments.

\section{1 $\gamma Z$ box corrections for $Q_{\text {weak }}$}

The $\Re e \square_{\gamma Z}^{V}$ contribution, given by Eq. (3.16), is plotted as a function of beam energy, $E$, in Fig. 6.1. In the upper panel, the contributions from Regions I, II and III are presented in conjunction with the total correction, while in the lower panel, the breakdown of the dominant Region I into its resonance and background components is shown. A summary of these contributions and their uncertainties at $Q_{\text {weak }}$ energy is given in Table. 6.1.

From these plots it is clear that at low energy $(E \lesssim 1 \mathrm{GeV})$, by far the largest contribution to the $\gamma Z$ box comes from the low- $Q^{2}$, low- $W^{2}$ Region I. This agrees well with the earlier work of Refs. [106, 107, 108, 109, 110]. The separation into the resonance and background parts is also instructive, with their central values approximately equal at $Q_{\text {weak }}$ energies and their uncertainties compatible. The shapes of the two contributions are, however, quite distinct with the (red dashed) resonance curve peaking at approximately $0.7 \mathrm{GeV}$ before tapering off as $E$ increases, while the background on the other hand, increases steadily with beam energy. Most of the resonance contribution comes from the $\Delta(1232)$ resonance, with the others only accounting for secondary amounts. The final contributions come from Regions II and III, with both giving only minor additions to $\Re e \square_{\gamma Z}^{V}$ at low energies. At $E=1.165 \mathrm{GeV}$, Region II accounts for approximately 10\%, while Region III accounts for even less at $\approx 6 \%$. This emphasises the fact that for $Q_{\text {weak }}$ it is most important to have an accurate description of the structure functions in the resonance region.

Although Table 6.1 divides the uncertainties into regions, we may also separate the $\Re e \square_{\gamma Z}^{V}$ error into background and resonance components. This is especially useful for comparing with the equivalent error breakdown in Ref. [110]. At $Q_{\text {weak }}$ energy we find,

$$
\Re e \square_{\gamma Z}^{V}=\left(5.57 \pm 0.21_{[\mathrm{bgd}]} \pm 0.29_{[\mathrm{res}]} \pm 0.02_{[\mathrm{DIS}]}\right) \times 10^{-3},
$$



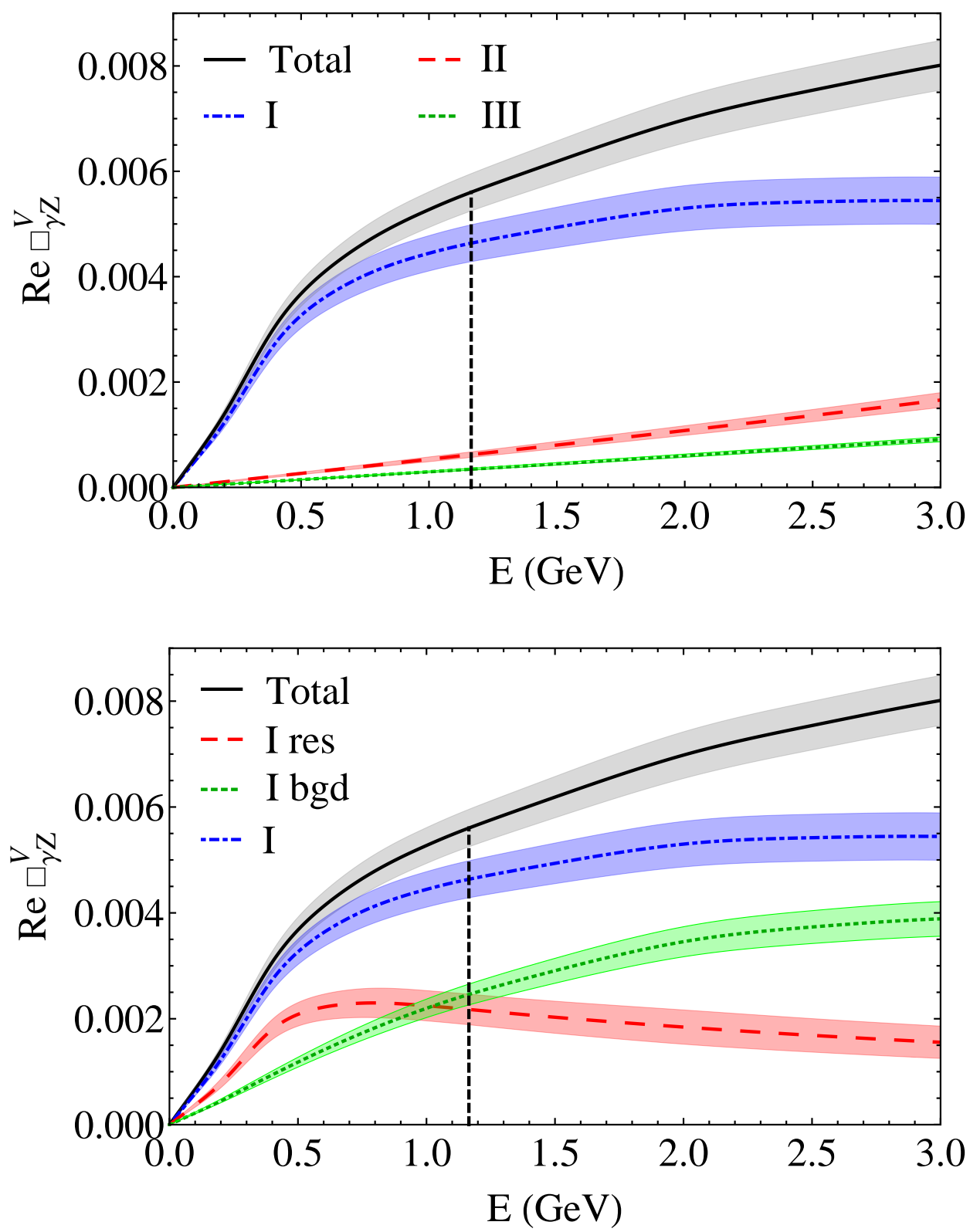

Figure 6.1: Energy dependence of the contributions to $\Re e \square_{\gamma Z}^{V}$ from the various regions in $Q^{2}$ and $W$ displayed in Fig. 5.1 in the AJM model (top), and the breakdown of Region I into its resonance and nonresonant background components (bottom). 


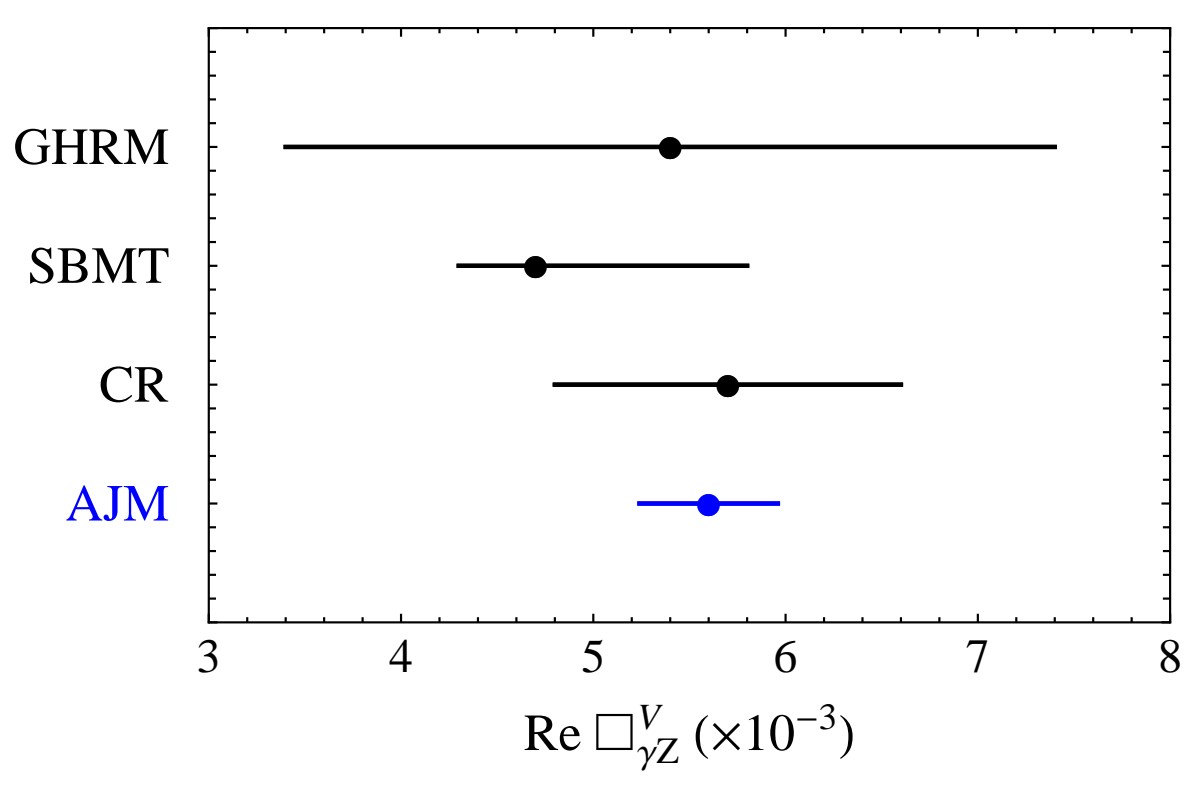

Figure 6.2: Comparison of the AJM model $\Re e \square_{\gamma Z}^{V}$ with earlier work.

where 'bgd' refers to the background error, 'res' the resonances' and 'DIS' the uncertainty coming from the deep inelastic region. Comparing with GHRM's calculation (Eq. 56 of Ref. [110]), the error on our resonances is about $\sim 40 \%$ smaller. This most likely reflects the difference between taking the extremal errors of the helicity amplitudes as GHRM have done, versus combining them in quadrature as we did. The largest difference between this work and GHRM is seen, however, in the uncertainty of the background, where the constrained continuum parameters have led to a background error which is roughly 9 times smaller than those in Ref. [110].

Combining these errors in quadrature we have,

$$
\Re e \square_{\gamma Z}^{V}=(5.57 \pm 0.36) \times 10^{-3}
$$

where the relative uncertainty on $\Re e \square_{\gamma Z}^{V}$ stays approximately constant with increasing energy, even though at large $E$ the contributions from Regions II and III become more important. As the structure functions have been constrained using global PDF fits, it is perhaps not surprising that the uncertainty remains largely energy independent.

In Fig. 6.2 we plot the total $\square_{\gamma Z}^{V}$ correction determined using the AJM model and compare it with the values calculated by the previous groups shown in Table 4.1 of Chapter 4. As can be seen, our central value agrees well with earlier determinations of $\Re e \square_{\gamma Z}^{V}$. The uncertainty of the AJM model is, on the other hand, significantly smaller, with the next smallest error more than twice the size. ${ }^{1}$

\footnotetext{
${ }^{1}$ While SBMT have a lower error on $\Re e \square_{\gamma Z}^{V}$ which is approximately the same as that in Eq. (6.2), the average of the SBMT's upper and lower uncertainties is more than twice that of the AJM model.
} 


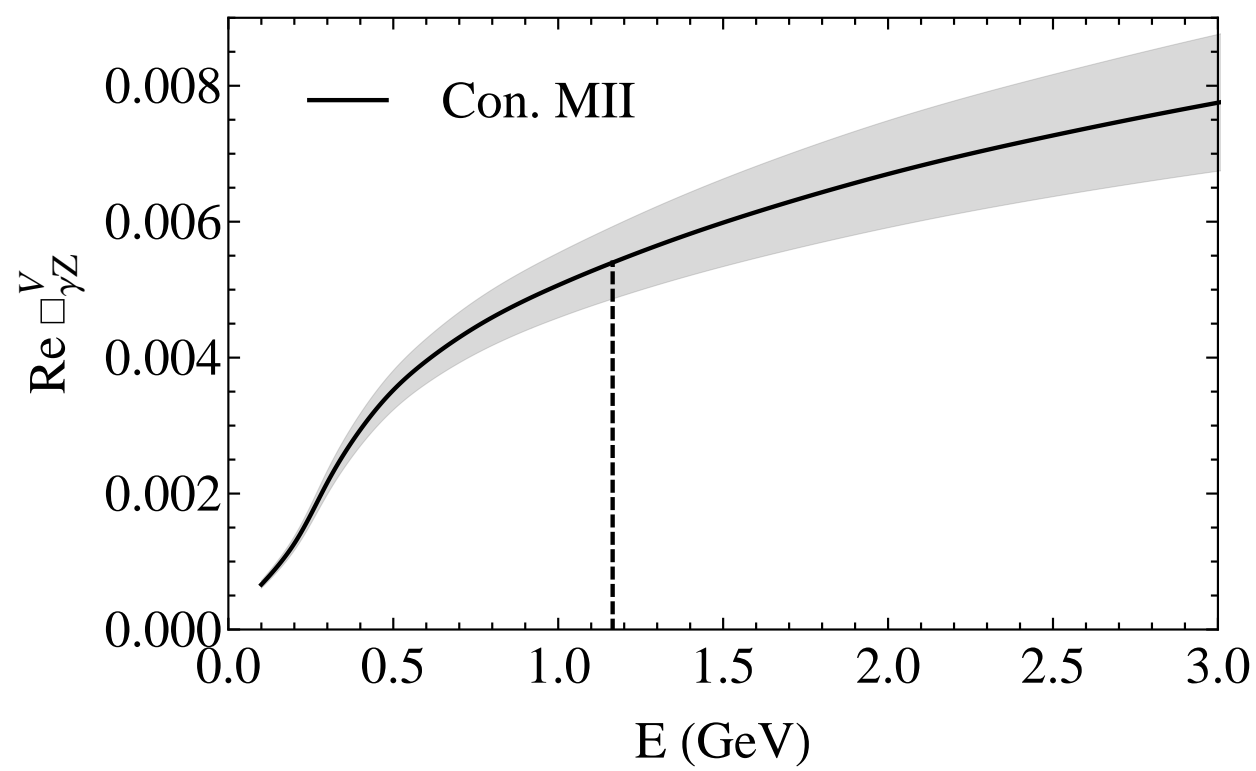

Figure 6.3: Energy dependence of the contributions to $\Re e \square_{\gamma Z}^{V}$ from a constrained version of Model II.

Although the AJM model provides the most accurate and precise determination of the $\gamma Z$ box correction, it is helpful to compare this value with an alternative version of GHRM's Model II. In this estimate, the structure functions of Region II of the AJM model are extended to all kinematics - as in Model II of Ref. [110]. Remember, however, that these extended structure functions have been constrained using the PDFs [141], before being used as inputs to the dispersion integral to calculate $\Re e \square_{\gamma Z}^{V}$. The results for this constrained Model II are shown in Fig. 6.3 where at $Q_{\text {weak }}$ energy we have,

$$
\Re e \square_{\gamma Z}^{V}=(5.40 \pm 0.54) \times 10^{-3} .
$$

The uncertainty here is $\sim 4$ times smaller than those determined by Gorchtein et al. Compared with the AJM model though, it is still slightly larger. Such a determination suggests what might occur should the information available from PDF fits and $e-d \mathrm{PV}$ scattering data be taking into account in the construction of GHRM's Model II.

\subsection{Predictions for parity-violating asymme- tries}

The constrained deuteron $\gamma Z$ structure functions may be used to further determine the parity-violating deep inelastic scattering asymmetry measured by the E08-011 experiment at JLab [56, 93]. The AJM predictions for the experiments' $Q^{2}$ values are plotted as a function of $W$ in Fig. 6.4. Since, as 

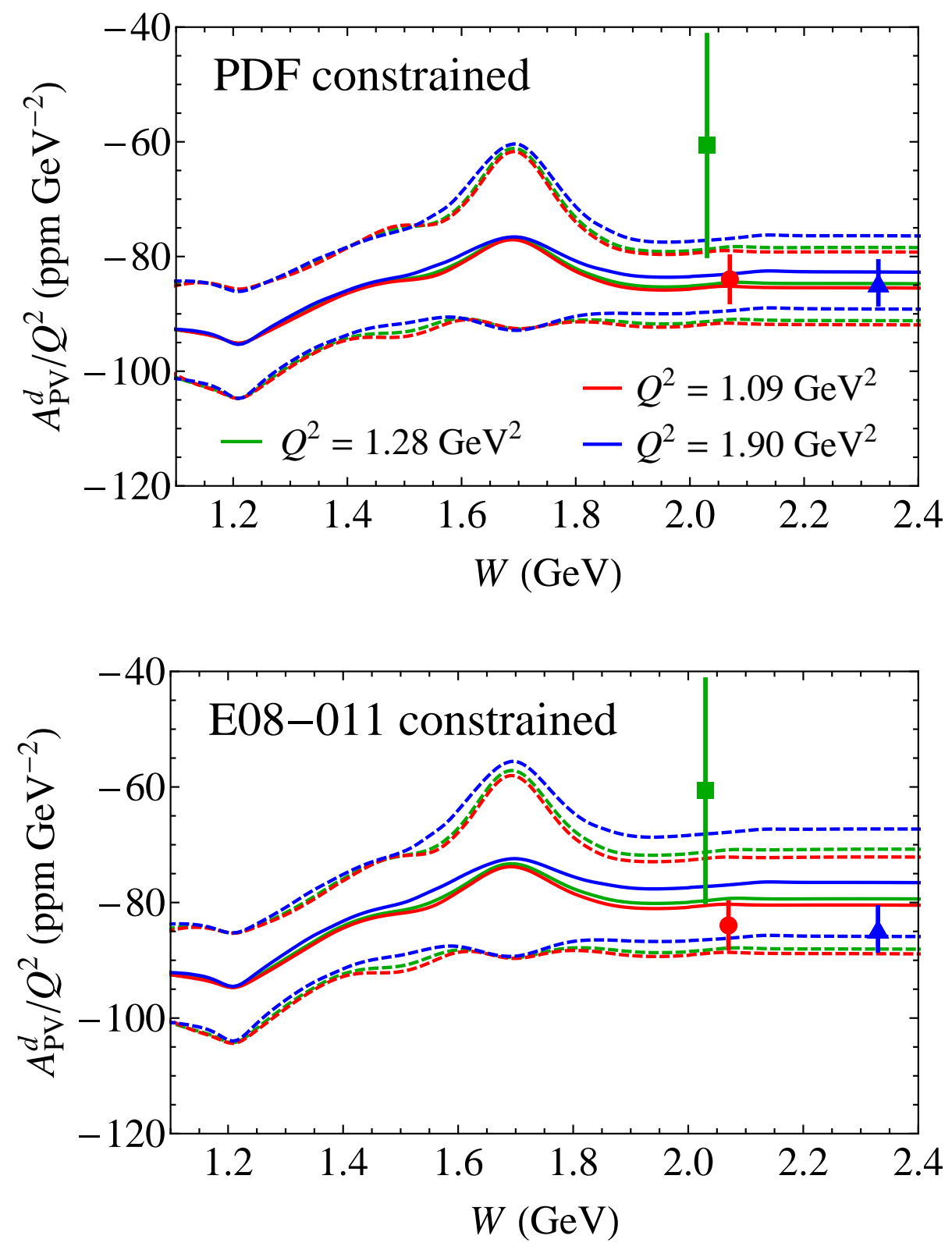

Figure 6.4: Comparison of the predictions for the PV deuteron asymmetry $A_{\mathrm{PV}}^{d} / Q^{2}$ as a function of $W$ (solid) for the DIS region kinematics of the Jefferson Lab E08-011 experiment $[93,154]$ at $Q^{2}=1.28 \mathrm{GeV}^{2}$ (green), $1.09 \mathrm{GeV}^{2}$ (red) and $1.90 \mathrm{GeV}^{2}$ (blue) (see also Table 5.2). The uncertainties (dashed) are computed in the AJM model with the continuum parameters $\kappa_{C}^{T, L}$ constrained by DIS structure functions (top), and by the E08-011 resonance region data (bottom). 


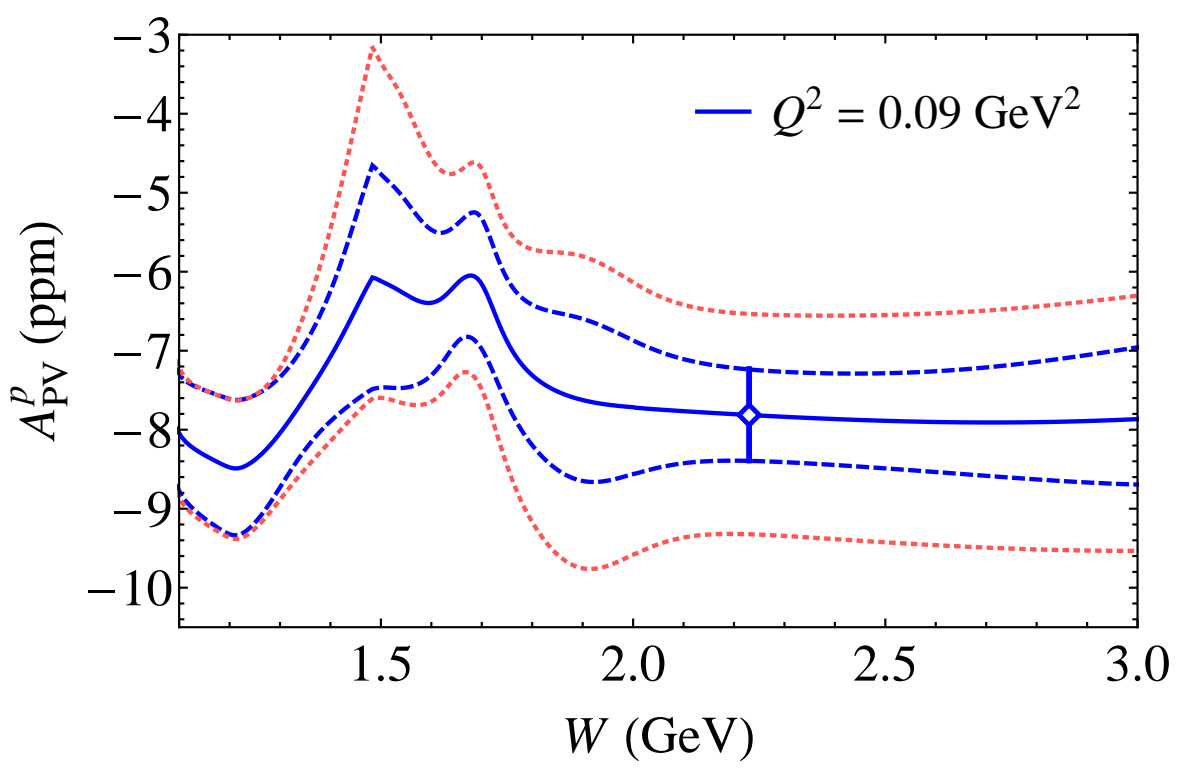

Figure 6.5: AJM model prediction for the proton parity-violating asymme$\operatorname{try} A_{\mathrm{PV}}^{p}$ as a function of $W$ for the $Q_{\text {weak }}$ inelastic measurement [153] at $Q^{2}=0.09 \mathrm{GeV}^{2}$ (solid line and open symbol). The AJM model uncertainties (dashed) are compared with those from the GHRM model with 100\% uncertainty on the continuum parameters (dotted).

discussed previously, we may constrain the structure functions using either the DIS parton distributions [141] or the resonance E08-011 data, both are displayed in this figure. For the numerical values of these predictions see Table 5.2 of Chaper 5. The experimental measurements from Ref. [93] are represent by the solid data points. Note that the uncertainties on AJM model curves include the total errors from the resonances and the background as before.

Comparing the two predictions, we see that the central values for the E08-011 constrained asymmetries are slightly higher than those given by the PDF constraints. This agrees with our previous findings in Figs. 5.10 and 5.11 for the resonance asymmetries. Also similar is the fact that the uncertainties are larger for the data constrained $A_{\mathrm{PV}}(d)$. The unconstrained structure functions - that is, what would be expected using the $100 \%$ error assumptions of Ref. [110] - on the other hand, give predictions approximately 4-5 times larger. Most importantly, the both methods give asymmetries which are in good agreement with the experimental results. Furthermore the PDF constrained curves more closely match the data points, which further confirms the reliability of our method in using parton distributions to restrict the $\gamma Z$ structure functions.

An additional data point will be available from the $Q_{\text {weak }}$ experiment which will measure the inelastic asymmetry for the proton at an invariant mass of $W=2.23 \mathrm{GeV}$. Since the $Q_{\text {weak }}$ asymmetry involves the proton structure 
functions, this will be particularly useful for constraining the $\Re e \square_{\gamma Z}^{V}$ uncertainty. In Fig. 6.5 we plot the AJM model prediction for $A_{\mathrm{PV}}^{p}$ together with an estimate its uncertainty. Numerically we have,

$$
A_{\mathrm{PV}}^{p}=(-7.8 \pm 0.6) \mathrm{ppm}
$$

for momentum transfer $Q^{2}=0.09 \mathrm{GeV}^{2}$. The structure functions with constrained $\kappa_{C}^{T, L}$, predict asymmetry uncertainties roughly two times smaller than the estimated using GHRM's model [110]. In order to be able to differentiate between the models, the experimental uncertainty would need to significantly smaller than that of GHRM, which is $\sim 16 \%$.

In the resonance region where $W \approx 1.5 \mathrm{GeV}$, the background contribution to the cross sections is small and the large uncertainty in the GHRM model is a direct result of taking extrema values for the helicity amplitudes rather than adding in quadrature. Using the more conventional method for combining uncertainties, the GHRM errors would almost be equal to the AJM model's at this point. Needless to say, additional measurements such as the $Q_{\text {weak }}$ inelastic measurement and higher energy deuteron PV asymmetries, will be invaluable for further constraining the interference structure functions and the $\Re e \square_{\gamma Z}^{V}$ correction.

\section{3 $Q_{W}^{p}$ at $11 \mathrm{GeV}$}

So far in this thesis, the AJM model has only been utilised for those experiments performed using Jefferson Lab's $6 \mathrm{GeV}$ polarised electron beam. The results, comparisons and predictions have been limited to the kinematic regions associated with this beam energy. Here we discuss some of the implications of the constrained structure functions relevant to a new range of measurements currently planned for the $12 \mathrm{GeV}$ upgrade to the JLab accelerator. These new experiments (such as MOLLER [55] and SoLID [57]) will result in even tighter constraints on the SM. In this section we will look at the MOLLER experiment which proposes to measure the PV asymmetry in elastic electron-electron scattering. What we are specifically interested in, are the $\gamma Z$ corrections to the $Q_{W}^{p}$ measurement - an important background arising from electron-proton scattering. Since one of the main theoretical uncertainties in the experiment is this $Q_{W}^{p}$ measurement, care is needed to ensure the correct evaluation of the $\square_{\gamma Z}^{V}$ contributions.

The experiment's set up involves scattering longitudinally polarised electrons from atomic electrons present in the energy shells of the (liquid) hydrogen target. The aim is to measure the electron's weak charge, $Q_{W}^{e}$, to $2.3 \%$ [55]. Such a determination would be equivalent to measuring the weak mixing angle, $\sin ^{2} \theta_{W}$, to $0.1 \%$ and would equal the precision of the two (different) measurements made at the $Z$-pole by LEP. Although the PDG value for $\sin ^{2} \theta_{W}$ is currently taken as the average of these two measurements, they in fact differ by $3 \sigma$ and were the MOLLER experiment to show either of these to be 

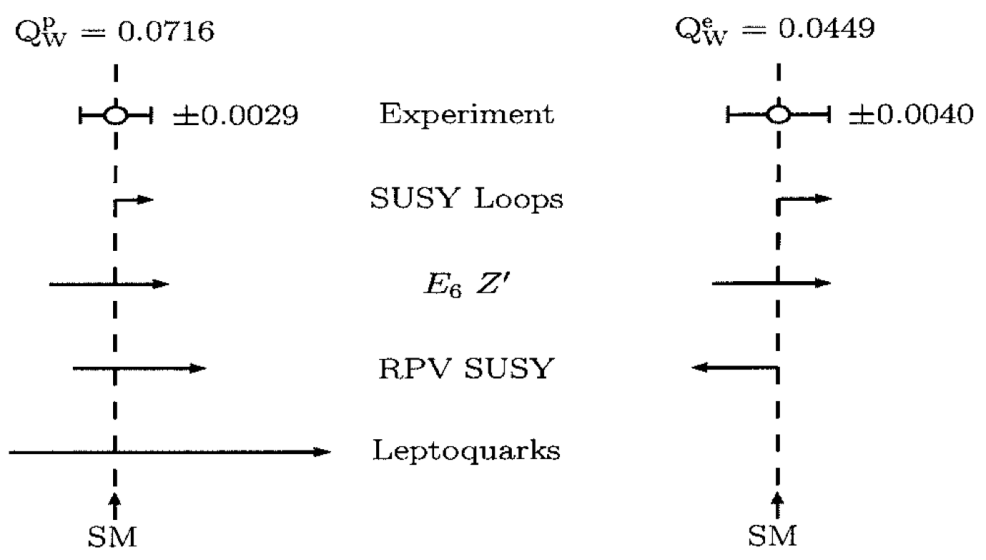

Figure 6.6: Effects of BSM physics on the theoretical values of the proton's and electron's weak charge [98].

correct, the running of $\sin ^{2} \theta_{W}$ (with respect to $Q$ ) would change markedly [55] from the SM expectation. Were the average at the $Z$-pole shown to be correct, however, the new measurement would still be important in constraining the possibility of new physics $[55,74,98]$. Given that the effects of certain BSM physics differ in how they alter the theoretical predictions of the proton's and electron's weak charge as illustrated in Fig. 6.6, this measurement is highly complementary to the $Q_{\text {weak }}$ experiment [98].

As for $Q_{\text {weak }}$, the asymmetry may be written as,

$$
A_{\mathrm{PV}}=\frac{\sigma_{+}-\sigma_{-}}{\sigma_{+}+\sigma_{-}} .
$$

Although here, $\sigma_{\lambda}$ is the cross section for the left-handed $(\lambda=-1)$ or righthanded $(\lambda=+1)$ beam electron scattering from the atomic electrons instead of protons. Once more the $A_{\mathrm{PV}}$ is dominated by the interference between the exchanges of $\gamma$ and $Z$. From Ref. [155] we have,

$$
A_{\mathrm{PV}}=m_{e} E \frac{G_{F}}{\sqrt{2} \pi \alpha} \frac{2 y(1-y)}{1+y^{4}+(1-y)^{4}} Q_{W}^{e},
$$

where we remind the reader that $\alpha$ is the electromagnetic structure constant, $E$ is the incoming electron's energy and $m_{e}$ is the mass of the electron. At the Born level, the electron's weak charge is defined as,

$$
Q_{W}^{e(\text { Born })}=-1+4 \sin ^{2} \theta_{W} .
$$

However, for the precision required by the MOLLER experiment, radiative corrections need to be included also. Unlike for $Q_{W}^{p}$, there is little controversy over the calculated $[156,157,158] Q_{W}^{e}$ radiative corrections.

An unavoidable background of the MOLLER experiment comes from the use of a hydrogen target. Not only will the incoming electrons scatter off the electrons, but they will also collide with the protons of the target, resulting in a PV background which depends on the proton's weak charge. The $Q_{\text {weak }}$ 

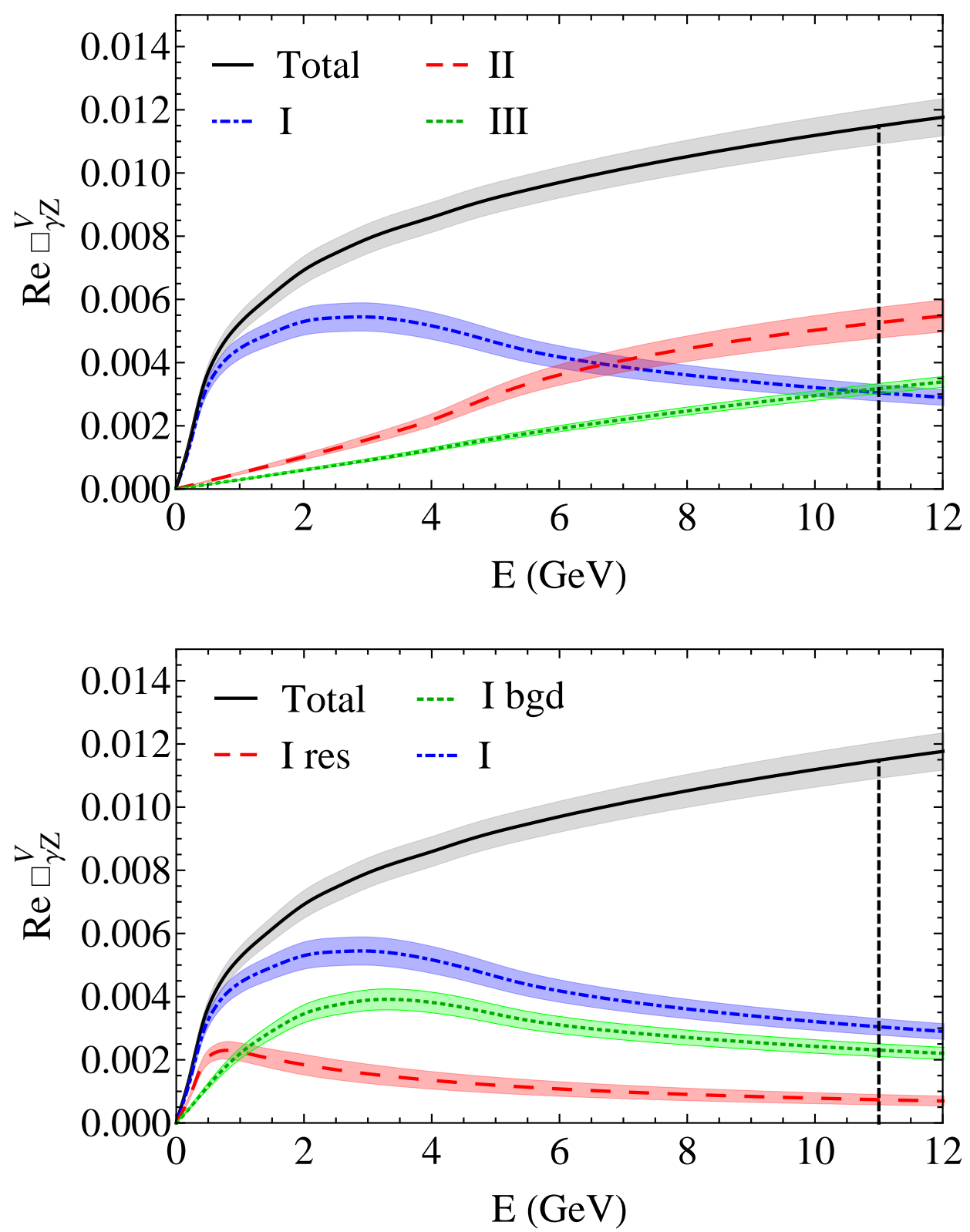

Figure 6.7: Energy dependence of the contributions to $\Re e \square_{\gamma Z}^{V}$ from the $W^{2}$ and $Q^{2}$ Regions I (blue dot-dashed line), II (red dashed line), and III (green dotted line), together with the total (black solid line). The lower panel shows the break up of Region I into resonance and background contributions as in Fig. 6.1. The vertical line indicates the energy at the MOLLER experiment. 
Table 6.2: Contributions to $\Re e \square_{\gamma Z}^{V}$ in the AJM model from Regions I, II and III at the kinematics of the $Q_{\text {weak }}(E=1.165 \mathrm{GeV})$ and MOLLER $(E=$ $11 \mathrm{GeV}$ ) experiments.

\begin{tabular}{lcc}
\hline \hline & $\Re e \square_{\gamma Z}^{V}\left(\times 10^{-3}\right)$ \\
Region & $Q_{\text {weak }}$ & MOLLER \\
\hline I & $4.64 \pm 0.35$ & $3.04 \pm 0.26$ \\
II & $0.59 \pm 0.05$ & $5.26 \pm 0.49$ \\
III & $0.35 \pm 0.02$ & $3.18 \pm 0.16$ \\
\hline Total & $5.57 \pm 0.36$ & $11.5 \pm 0.6$ \\
\hline \hline
\end{tabular}

experiment should determine $Q_{W}^{p}$ to an accuracy level of $4 \%$ at a beam energy of $1.165 \mathrm{GeV}$. As we have pointed out, the $\gamma Z$ box contribution is particularly important to the theoretical calculation of $Q_{W}^{p}$. Although for $Q_{\text {weak }}$ the $\square_{\gamma Z}^{V}$ contributes an $\approx 7 \%$ correction, this grows significantly with increasing energy and at the energy of MOLLER $(E=11 \mathrm{GeV})$, we therefore expect this contribution to be substantially larger.

Given the experimental error budget for the weak charge of the proton is $\lesssim 4 \%$, it is imperative that the $\gamma Z$ box correction is accurately determined and any uncertainties well accounted for at these much larger energies. While there has to date been no determination of $\square_{\gamma Z}^{V}$ for these kinematics, by extending the work of previous sections we have a reliable way of calculating this important correction.

In Fig. 6.7 we show the energy dependence of $\Re e \square_{\gamma Z}^{V}$ up to $12 \mathrm{GeV}$ calculated using the AJM model. As in Fig. 6.1 the break down of the individual kinematic regions' contributions has also been included. In Table 6.2 these are compared with the equivalent $Q_{\text {weak }}$ values. Looking at the total correction for the MOLLER experiment, the $\Re e \square_{\gamma Z}^{V}$ correction is approximately twice that of $Q_{\text {weak }}$ and now, almost a third of the tree level $\sin ^{2} \theta_{W}$. Additionally, the contributions from each of the individual kinematic regions differs dramatically. Before, at $1.165 \mathrm{GeV}$, Region II accounted for about $10 \%$ of the total contribution, while now at $E=11 \mathrm{GeV}$, it is close to $50 \%$. On the other hand, Region I which use to be more than $80 \%$ of $\Re e \square_{\gamma Z}^{V}$ now accounts for only a third of the correction. Region III, while contributing more here than for $Q_{\text {weak }}$, remains relatively less important, yielding about the same as the low- $Q^{2}$, low- $W^{2}$ region.

\section{Model dependence}

Although the AJM model gives the best determination of $\Re e \square_{\gamma Z}^{V}$ and its uncertainties currently available using constraints from PDFs and other experimental data, it is necessary to ensure that any model dependence of the correction is accurately taken into account. Here we examine the effects 

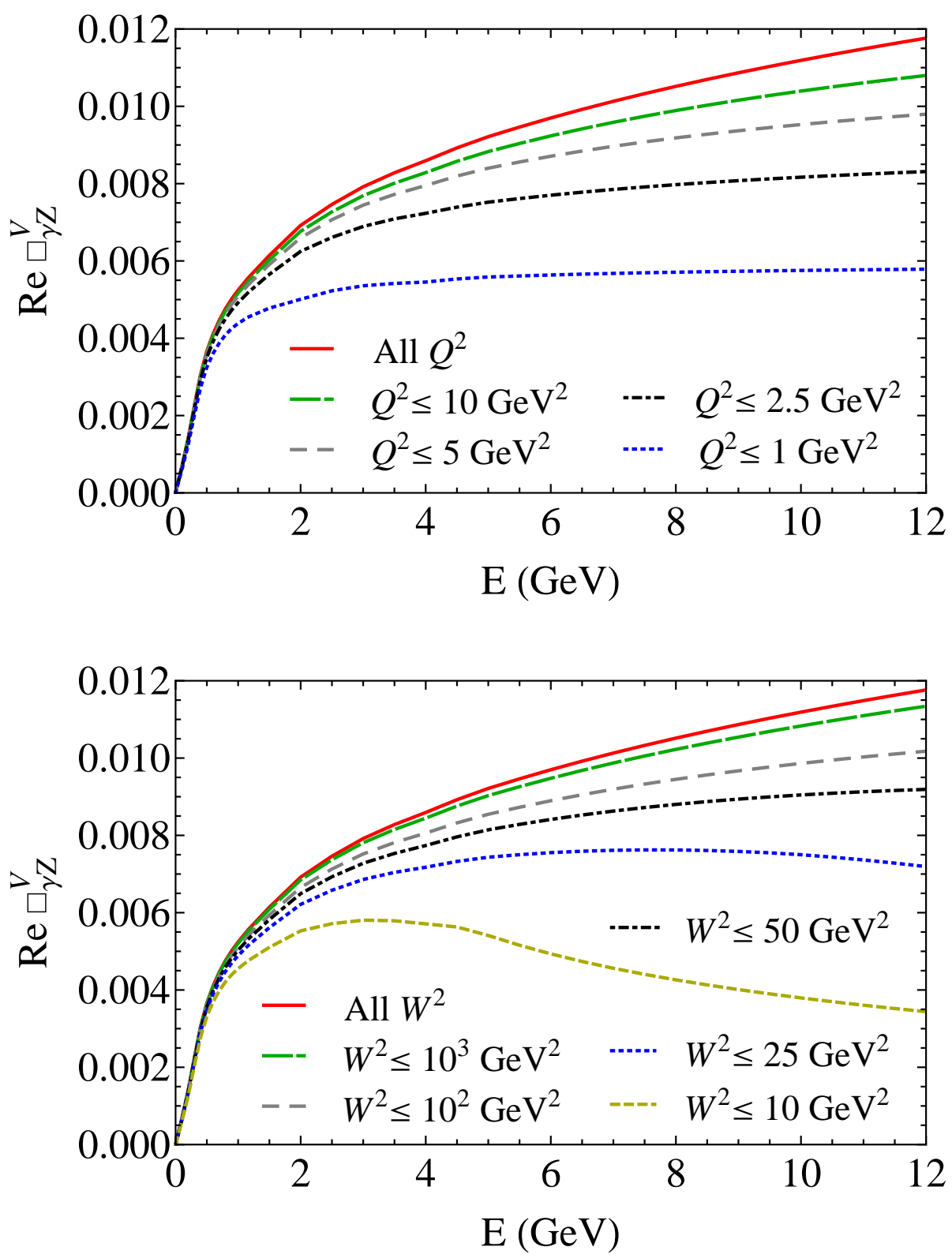

Figure 6.8: Contributions to $\Re e \square_{\gamma Z}^{V}$ from various kinematic regions in $Q^{2}$ (top) and $W^{2}$ (bottom), as a function of the energy $E$. 

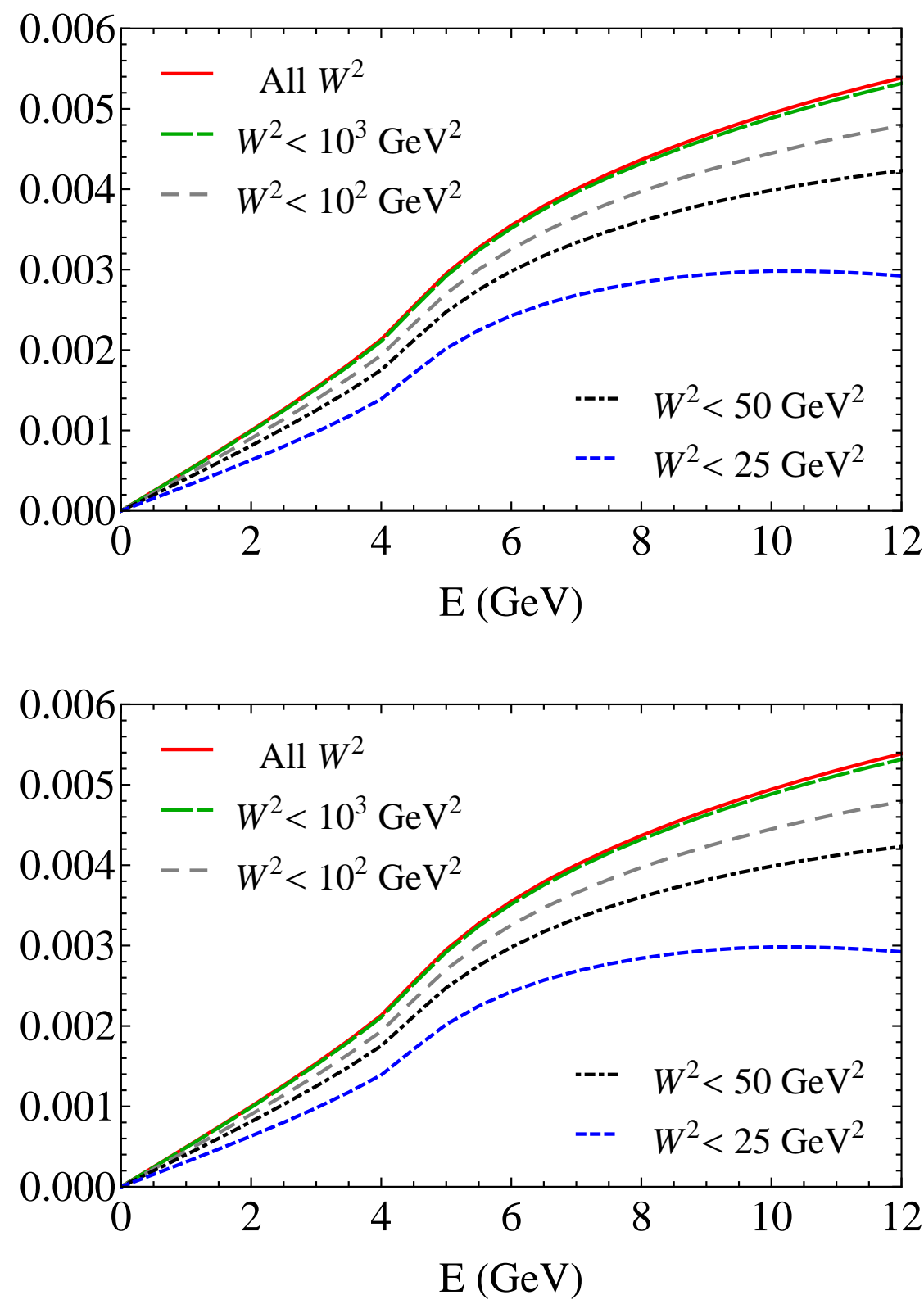

Figure 6.9: Contributions to $\Re e \square_{\gamma Z}^{V}$ from various kinematic regions of $Q^{2}$ (top) and $W^{2}$ (bottom) in Region II. 
the choice of model might have on the most important Region II contribution where the VMD+Regge description is used. We also investigate the kinematic dependence of the continuum parameters $\kappa_{C}^{i}$ and how this might alter the calculation of $\Re e \square_{\gamma Z}^{V}$.

To find the kinematic regions most relevant to the evaluation, we divide the $Q^{2}-W^{2}$ plane into separate intervals in $Q^{2}$ and $W^{2}$. The results for these divisions are illustrated in Fig. 6.8. Similar plots examining different intervals specifically within Region II are shown in Fig. 6.9. For the total contribution, at $E \lesssim 1 \mathrm{GeV}$, the $Q^{2}<1$ region tends to dominate. By the time $E$ increases to $\approx 10 \mathrm{GeV}$, however, this contribution makes up only $\sim 50 \%$ of $\Re e \square_{\gamma Z}^{V}$. Alternatively, if we examine the $W^{2}$ regions, most of contribution comes from below $W^{2} \sim 9 \mathrm{GeV}$ where the resonance region lies. As the energy increases, the higher $W^{2}$ region becomes significantly more important. For example, the $W^{2}>10 \mathrm{GeV}^{2}$ interval comprises almost two thirds of the total box contribution at $E \approx 10 \mathrm{GeV}$.

In order to test the model dependence of Region II, we examine several alternative models for the $\gamma Z$ interference structure functions. Each of these models is based on electromagnetic parametrisations of cross section data in the kinematic regions relevant to Region II and rely on different physical mechanisms of the scattering events. With the electromagnetic parametrisations mostly been discussed previously in Chapter 4 , we simply summarise here the $\gamma Z$ models used in the comparison:

\section{Modified Regge model (MRM)}

While this follows Sibirtsev et al. [108] in the use of the Capella et al. parametrisation [133] for the electromagnetic cross sections, for the interference structure functions, we use the $\gamma Z / \gamma \gamma$ ratio of Eq. (4.32) to modify the $F_{1,2}^{\gamma \gamma}$ instead of the leading-twist parton distributions that SBMT employ. Unlike previous models, where the cross sections could be split into separate resonance and background components and each part be modified independently, the Capella et al. structure functions can only be transformed in their entirety. This may at first seem ad hoc, since each individual resonance will be rotated by the same amount in this method, however, as the resonance piece is negligible in Region II, the total cross sections $\sigma_{T, L}$ are effectively given by just their backgrounds, i.e.

$$
\sigma_{T, L} \approx \sigma_{T, L}^{(\mathrm{bgd})}
$$

\section{CDP model}

The colour dipole parametrisation of the electromagnetic cross sections $[127$, 129] has been discussed in detail in Section. 4.3. As mentioned, this is the basis of Model I used in Gorchein et al. [110]. Instead of using the VMD to compute the $\kappa_{V}$ and $\kappa_{C}^{i}$ values in the ratio of Eq. (4.32), these are determined using the ratios of electric quark charges. This results in a constant scaling 


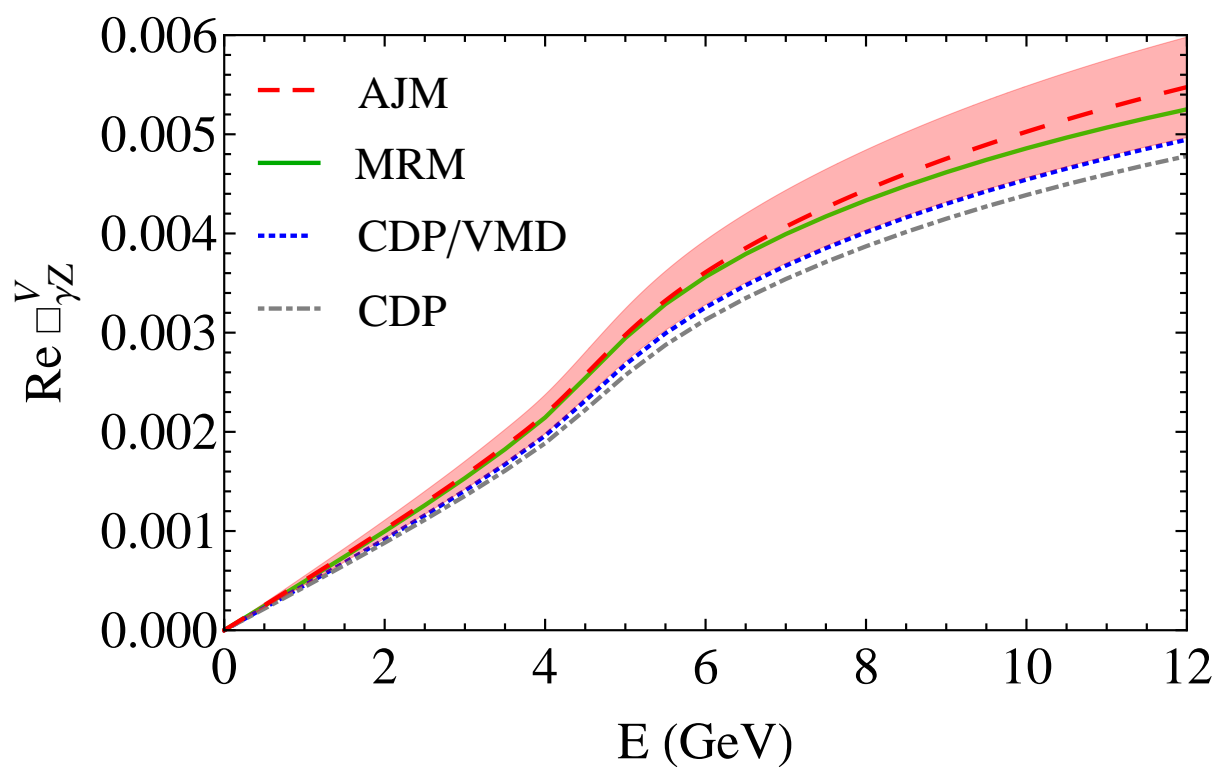

Figure 6.10: Contribution of Region II to $\Re e \square_{\gamma Z}^{V}$ as a function of energy using various models for the $\gamma Z$ structure functions: AJM (red dashed line), MRM (green solid line), CDP/VDM (blue dotted line), and CDP (gray dot-dashed line).

factor for the $\sigma_{i}^{\gamma Z} / \sigma_{i}^{\gamma \gamma}$ ratio [110],

$$
\frac{\sigma_{i}^{\gamma Z}}{\sigma_{i}^{\gamma \gamma}}=\frac{9}{5}-4 \sin ^{2} \theta_{W} .
$$

In this work we use an updated version of the CDP parametrisation of the transverse and longitudinal cross sections from Ref. [159].

\section{$C D P / V M D$ model}

Finally, we use the electromagnetic cross sections of Cvetic et al. [127, 129] and modify them into their interference analogues using the $\gamma Z / \gamma \gamma$ ratio of Eq. (4.32). We point out that for both this model and the CDP parametrisation, the structure functions have only been given for $W^{2}<1000 \mathrm{GeV}^{2}$. Since it is clear from Fig. 6.8 that the contribution to $\Re e \square_{\gamma Z}^{V}$ from $W^{2}>1000 \mathrm{GeV}^{2}$ is minimal this is not a problem. However, in order to get as close as possible to the correct Region II contribution, we scale the CDP and CDP/VMD results by the same amount in going from $W^{2}=1000 \mathrm{GeV}^{2}$ to $W^{2}=\infty$ using the AJM model i.e.,

$$
\operatorname{Mod}_{\infty}=\operatorname{Mod}_{1000} \times \frac{\mathrm{AJM}_{\infty}}{\mathrm{AJM}_{1000}}
$$

where 'Mod' refers to either the CDP or CDP/VMD model contribution to Region II and the AJM contributions (to Region II) up to $W^{2}=1000 \mathrm{GeV}^{2}$ and $W^{2}=\infty$ are given by $\mathrm{AJM}_{1000}$ and $\mathrm{AJM}_{\infty}$ respectively. 


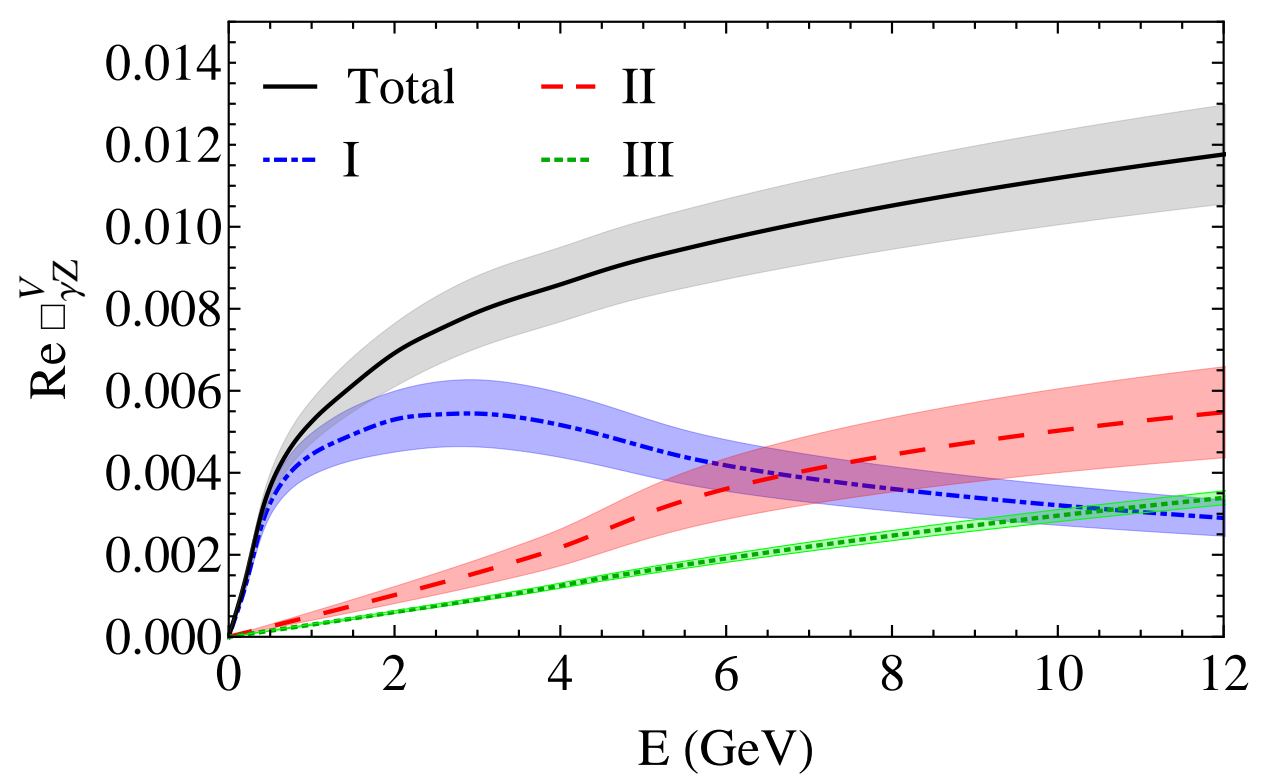

Figure 6.11: A plot showing the $\Re e \square_{\gamma Z}^{V}$ contribution and associated errors when the continuum parameter $\kappa_{C}^{T}$ is a linear function of momentum transfer for $0<Q^{2}<2.5 \mathrm{GeV}^{2}$.

In Fig. 6.10 we show the results of using these additional models which incorporated different physics in the $\gamma Z$ structure functions and compare with the AJM model of Ref. [90] in Region II. Looking at the AJM and MRM model determinations, we can see that these show excellent agreement over the full range of energies given. The $\mathrm{CDP}$ and $\mathrm{CDP} / \mathrm{VMD}$ models' contributions are, on the other hand, slightly less than the AJM model's. While the errors associated with the AJM model take into account the MRM and CDP/VMD parametrisations, the CDP results fall just outside the lower error band in Fig. 6.10. We take into account this model dependence in the Regge region by including an additional uncertainty found by taking the difference between the central value of the AJM model and the outer most CDP model. This is then combined with the uncertainty arising from the AJM parametrisation by adding the two contributions in quadrature.

A source of additional error to $\Re e \square_{\gamma Z}^{V}$ could come from the continuum parameters' dependence on the invariant mass. In the AJM model, the fitted values took into account possible $W^{2}$ dependence by matching the $\gamma Z / \gamma \gamma$ ratio over the range $W^{2}=4 \mathrm{GeV}^{2}$ to $13 \mathrm{GeV}^{2}$. Any resulting variation was included in the final uncertainties. If the $W^{2}$ range is increased to $4 \leq W^{2} \leq 1000 \mathrm{GeV}^{2}$, the fit gives,

$$
\kappa_{C}^{T}=0.86 \pm 0.24, \quad \kappa_{C}^{L}=-1.3 \pm 2.3
$$

which within errors, agrees with the previous determinations of $\kappa_{C}^{T}=0.65 \pm$ 0.14 and $\kappa_{C}^{L}=-1.3 \pm 1.7$ [90]. While the new values for $\kappa_{C}^{i}$ result in structure functions which differ from those used previously, the errors assigned to the 


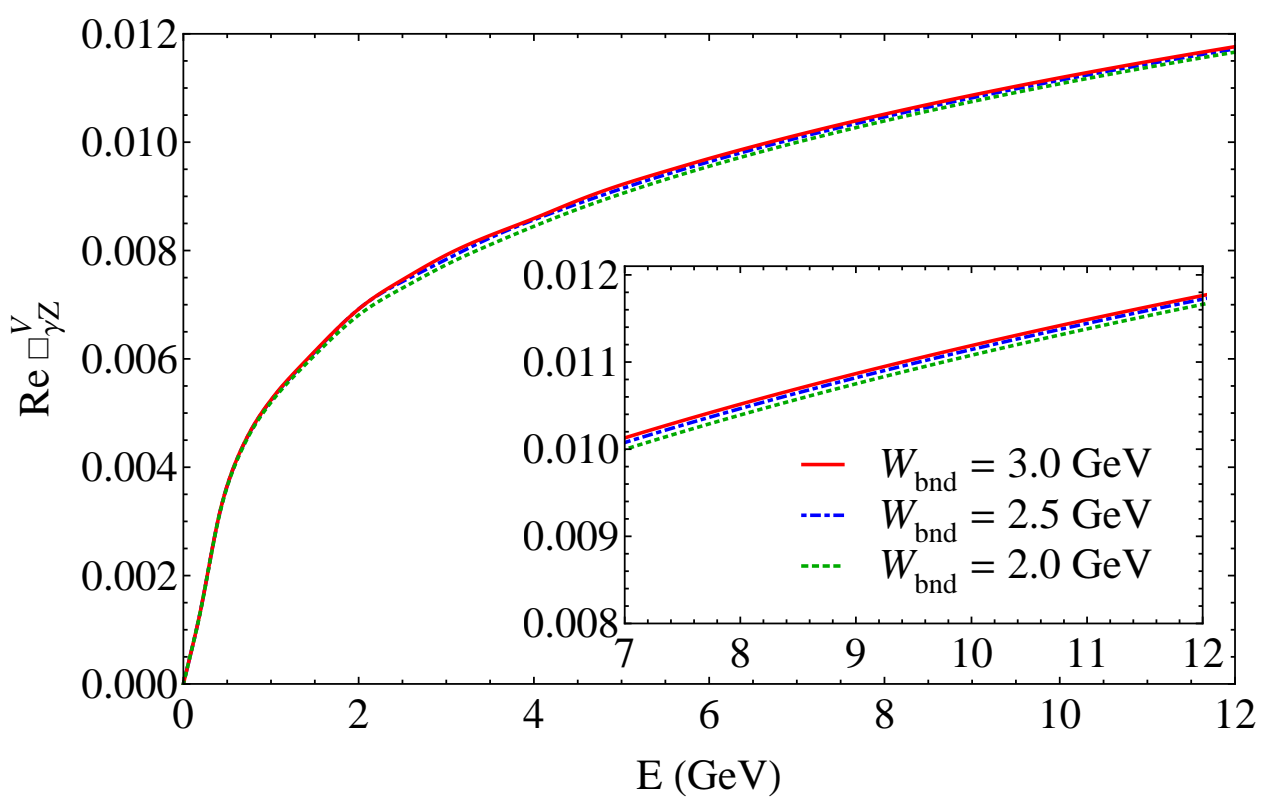

Figure 6.12: The $\Re e \square_{\gamma Z}^{V}$ contribution as it depends on the boundaries between Regions I and II.

AJM model structure functions are large enough to cover these alternative $F_{i}^{\gamma Z}$. As the additional error associated with the $W^{2}$ dependence of $\kappa_{C}^{i}$ is negligible, no further error needs to be added to our evaluation.

In a similar vein, the continuum parameters may also show some dependence on the momentum transfer $Q^{2}$. Since we constrain the $\kappa_{C}^{i}$ at $Q^{2}>2.5$, the only place where this $Q^{2}$-dependence might occur is in the region $0 \leq Q^{2} \leq$ $2.5 \mathrm{GeV}^{2}$. To estimate possible $Q^{2}$-dependence, we use the AJM values for $\kappa_{C}^{\bar{i}}$ for $Q^{2} \geq 2.5 \mathrm{GeV}^{2}$ while for $Q^{2}<2.5$ we linearly increase the uncertainties to $100 \%$ at $Q^{2}=0$. The energy dependence of these modified constraints is illustrated in Fig. 6.11. Numerically we find the error increases to $\pm 0.59 \times 10^{-3}$ at the $Q_{\text {weak }}$ energy, while for MOLLER there is a $200 \%$ increase, with the total uncertainty becoming $\pm 1.2 \times 10^{-3}$. Although these errors are still within the budgeted uncertainty, we believe they are overly conservative and therefore in practice, take the errors on $\kappa_{C}^{T}$ to be $Q^{2}$ independent.

One last area which may artificially effect the result of $\Re e \square_{\gamma Z}^{V}$ is the use of fixed boundaries between the different regions. Figure 6.12 illustrates, however, the fact that the dependence on the position of the boundaries is negligible. Although not shown, we also tested the boundary-dependence of the $Q^{2}$ borders by lowering the DIS region limit from $Q^{2}=2.5 \mathrm{GeV}$ to $Q^{2}=1 \mathrm{GeV}$ and again, found no significant change in the results.

Using the PDF-constrained AJM to calculate the $\Re e \square_{\gamma Z}^{V}$ correction at MOLLER energies, we see that the relative contributions from the various regions differ significantly from those evaluated at the $Q_{\text {weak }}$ experiment's energy. Particularly important is the increase in the Region II contribution from about $10 \%$ to $\sim 50 \%$. Including additional errors arising from the model de- 
pendence of the Region II contribution, we estimate the total $\Re e \square_{\gamma Z}^{V}$ correction at $11 \mathrm{GeV}$, to be

$$
\Re e \square_{\gamma Z}^{V}=(11.5 \pm 0.6 \pm 0.6) \times 10^{-3},
$$

where the first error includes the previous uncertainties in the AJM model, and the latter comes from the additional model dependence discussed in this section. Of course, any future experiments would help to lower this uncertainty still further.

With the current level of precision available using the AJM model, the proton's (effective) weak charge grows from $0.0757 \pm 0.0007$ at $E=1.165 \mathrm{GeV}$ to $0.0814 \pm 0.0010$ at $E=11 \mathrm{GeV}$. A necessary inclusion in these $Q_{W}^{p}$ effective values is the vector electron, axial-vector hadron coupling piece, $\square_{\gamma Z}^{A}$. Extending the previous work of Refs. [101, 102], this term decreases from $0.0037(2)$ at $E=1.165 \mathrm{GeV}$ to $0.0035(2)$ at $E=11 \mathrm{GeV}$ [103]. Since the current background estimate from PV electron-proton scattering is around $8 \%$ for the MOLLER experiment, the uncertainty from $Q_{W}^{p}$ is $\approx 0.1 \%$. While still significant, this value is well within the budgeted $0.3 \%$ given in the MOLLER proposal.

\section{4 $A_{\text {PVDIS }}$ for the proton at $11 \mathrm{GeV}$}

As well as the background coming from elastic ep scattering, the MOLLER experiment will measure the inelastic cross section. Although this cross section is smaller than the elastic background by an order of magnitude, because it is not suppressed by a factor of $1-4 \sin ^{2} \theta_{W}$ as in the elastic case, it will still contribute a significant amount to the overall asymmetry. In this section we use the AJM model to estimate this important, additional background.

The proton parity-violating asymmetry given in Section 5.3,

$$
\begin{aligned}
& A_{\mathrm{PV}}=\left(\frac{G_{F} Q^{2}}{2 \sqrt{2} \pi \alpha}\right) \frac{x y^{2} F_{1}^{\gamma Z}+\left(1-y-\frac{x^{2} y^{2} M^{2}}{Q^{2}}\right) F_{2}^{\gamma Z}+\frac{g_{V}^{e}}{g_{A}^{e}}\left(y-\frac{1}{2} y^{2}\right) x F_{3}^{\gamma Z}}{x y^{2} F_{1}^{\gamma \gamma}+\left(1-y-\frac{x^{2} y^{2} M^{2}}{Q^{2}}\right) F_{2}^{\gamma \gamma}},
\end{aligned}
$$

is plotted using the AJM model $\gamma Z$ structure functions in Fig. 6.13, where for the MOLLER experiment, the momentum transfer is $Q^{2} \approx 0.004 \mathrm{GeV}^{2}$. As in the plots of the $\gamma Z$ structure functions, the matching between Regions I and II is excellent. For $W<2 \mathrm{GeV}$, the resonance structure of the cross sections is clearly evident in the asymmetry plotted. At larger $W$, the resonances become negligible and the asymmetry is mostly constant at $\approx 85 \mathrm{ppm} / \mathrm{GeV}^{2}$. Although the uncertainty grows slightly with $W$, for the most part it stays at $\approx 7 \%$ in our model. 


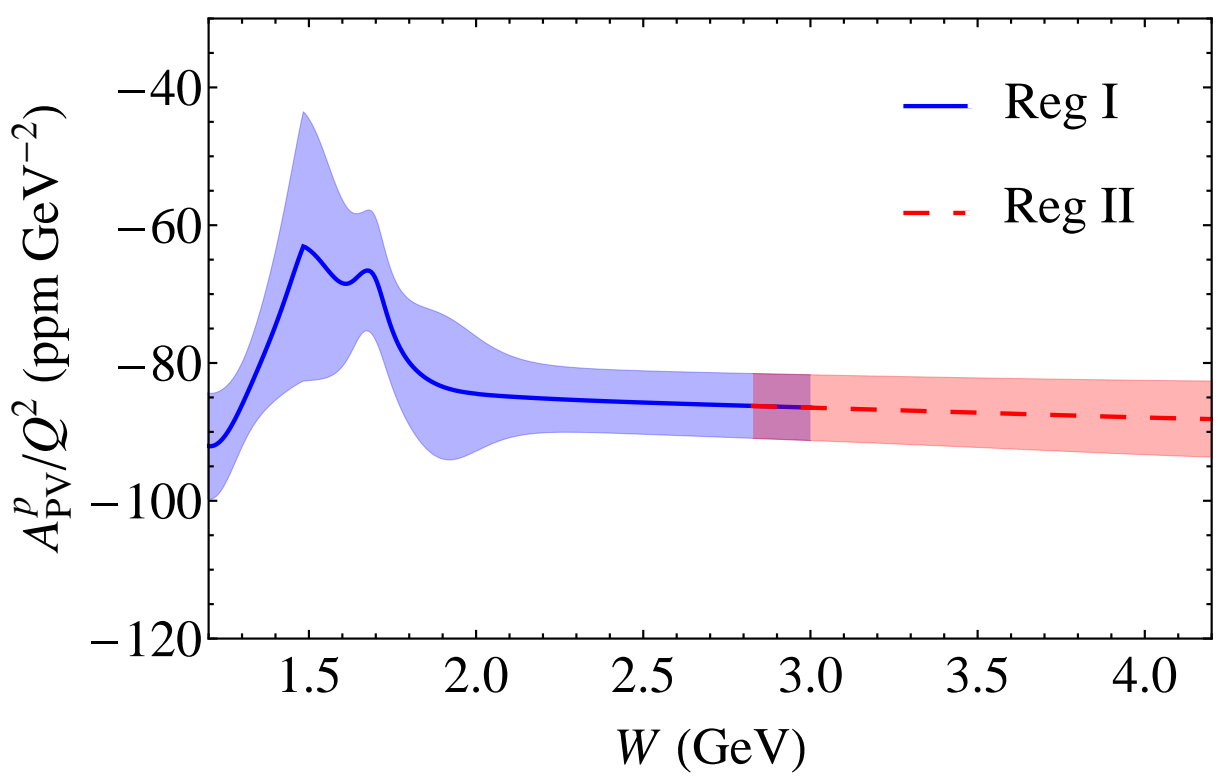

Figure 6.13: PV asymmetry $A_{\mathrm{PV}}^{p}$ for inelastic ep scattering, scaled by $1 / Q^{2}$, at $E=11 \mathrm{GeV}$ in the AJM model, showing the matching of the contributions from Region I (blue solid line) and Region II (red dashed line). The typical momentum transfer relevant to the MOLLER experiment is $\approx 0.004 \mathrm{GeV}^{2}$.

In the previous section, we investigated what would happen to the magnitude of the uncertainties were the continuum parameters $\kappa_{C}^{i}$ to depend on $Q^{2}$ in the region $0 \leq Q^{2}<2.5 \mathrm{GeV}^{2}$. Here, increasing the uncertainties linearly from the AJM values at $2.5 \mathrm{GeV}^{2}$ to $100 \%$ at the real photon point results in the relative error on the inelastic $A_{\mathrm{PV}}$ growing to approximately $25 \%$ for the MOLLER experiment. In such a scenario, additional data would be needed to further constrain the model, and it will be necessary for the MOLLER experiment to do so directly within their own setting. Given that the earlier SLAC E158 experiment was able to constrain their background to better than $20 \%$ [84], this should not be overly difficult.

Although PV inelastic scattering includes other standard radiative corrections that have not been included here, but which might alter the $W$ dependence shown in Fig. 6.13 and would therefore also need to be included in the final estimate of the background PV inelastic asymmetry, for the requirements of illustrating the characteristics of the asymmetry (together with the magnitude of the uncertainty) our analysis suffices. More detailed analyses would be necessary further along in the experimental process.

In summary, we have determined the energy dependence of the $\square_{\gamma Z}$ radiative correction to the elastic $\mathrm{PV}$ asymmetry to a precision level of about $\approx 7 \%$ needed for the MOLLER experiment at $E=11 \mathrm{GeV}$. Unlike in the $Q_{\text {weak }}$ experiment, where the resonance region dominates the final box correction, 
at energies relevant to MOLLER, the region with the largest contribution is Region II where Regge physics is most appropriate. By examining additional uncertainties arising from the model dependence of the interference structure functions in this region and including them in the overall uncertainty we find the proton's effective weak charge to be $0.0814 \pm 0.0010$ at $11 \mathrm{GeV}$. With these uncertainties, the $Q_{W}^{p}$ background is known precisely enough that it remains well within the experimental error budget.

Furthermore, we have used the AJM structure functions to calculate the magnitude of the inelastic ep asymmetry and the size of its uncertainty. Although the errors arising from the AJM model are small enough to remain within the experimental budget, using a more conservative $Q^{2}$-dependent $\kappa_{C}^{T}$ results in the conclusion that an experimental monitoring of this background will be needed for MOLLER. On the other hand, such a measurement could also be used as an additional constraint on the parameters of the AJM model, thus decreasing the uncertainties in the model further.

In the next two chapters we make use of the AJM model of the structure functions to study other nonperturbative effects. Chapter 7 uses the electromagnetic parametrisation to study the electric and magnetic polarisabilities of the proton, while in Chapter 8 we look at the higher-twist contributions to the nucleon structure function moments. 


\section{Electric and magnetic polarisabilities of the proton}

The interference structure functions have been used in the analysis of the proton's 'weak' properties. In a similar manner, the $\gamma \gamma$ structure functions can be utilised to determine the electromagnetic character of the proton. In this chapter we use the electromagnetic parametrisations upon which the AJM $\gamma Z$ expressions are based to determine the electric and magnetic polarisabilities of the proton. Although we limit ourselves to the polarisabilities of the proton here [160], such a study could also be done for the neutron. ${ }^{1}$ Since the various models have already been addressed in the development of the AJM model, we may proceed directly to the necessary background formalism.

\subsection{The generalised Baldin sum rule}

Polarisabilities occur in composite systems because the photon's electric and magnetic fields alter the dynamics the charged constituents resulting, even in neutral targets, in current and charge multipoles [161]. The parameters which describe this response in nucleons are the electric $(\alpha)$ and magnetic $(\beta)$ polarisabilities. Intuitively, $\alpha$ and $\beta$ characterise the rigidity of the nucleon and encode information regarding the electromagnetic charges and interactions of the internal constituents [161]. For structureless objects the values of $\alpha$ and $\beta$ vanish. The spin-dependent polarisabilities also play an important role in understanding the electromagnetic properties of the proton.

The polarisabilities $\alpha$ and $\beta$ are defined in the context of the expansion of the Compton amplitude at low energies, with the sum being the part of the electromagnetic polarisability which does not have its helicity flipped [162]. The static polarisabilities are related to the unpolarised photoabsorption cross sections by the Baldin sum rule - the unpolarised analogue of the Gerisamov-

\footnotetext{
${ }^{1}$ Difficulties arising from the (relative) instability of the neutron mean that experiments use deuterium instead. This results in much larger uncertainties on the neutron's electromagnetic polarisabilities.
} 
Drell-Hearn sum rule $[163,164]$ — which is given as $[165,166]$,

$$
\alpha+\beta=\frac{1}{4 \pi^{2}} \int_{\nu_{\pi}}^{\infty} \frac{\sigma_{1 / 2}+\sigma_{3 / 2}}{\nu^{2}} d \nu
$$

where $\sigma_{\lambda}$ is the cross section for the production of $\lambda=1 / 2$ and $3 / 2$ helicity states, $\nu$ is the photon energy and $\nu_{\pi}$ the pion production threshold. The sum rule establishes a relation between the static and dynamic properties of the nucleon and allows for the extraction of $\alpha+\beta$ from precision measurements of real Compton scattering [162].

Additional insight into the polarisation density is gained by studying the $Q^{2}$-dependence of $\alpha$ and $\beta$. Drechsel et al. used dispersion relations to extend Eq. (7.1) to the case of virtual Compton scattering [167]. This generalised Baldin sum rule [167] provides a way of determining the polarisabilities' dependence on the momentum transfer via radiative electron scattering. Explicitly, it is written [167],

$$
\begin{aligned}
\alpha\left(Q^{2}\right)+\beta\left(Q^{2}\right) & =\frac{8 \alpha_{\mathrm{em}} M}{Q^{4}} \int_{0}^{x_{\pi}} x F_{1}^{\gamma \gamma}\left(x, Q^{2}\right) d x, \\
& =8 \alpha_{\mathrm{em}} M \int_{W_{\pi}^{2}}^{\infty} \frac{F_{1}^{\gamma \gamma}\left(W^{2}, Q^{2}\right)}{\left(W^{2}-M^{2}+Q^{2}\right)^{3}} d W^{2},
\end{aligned}
$$

where $M$ is the mass of the proton, $\alpha_{\mathrm{em}}$ is the fine structure constant, $x=$ $Q^{2} /\left(W^{2}-M^{2}+Q^{2}\right)$ is the Bjorken scaling variable and $W$ the invariant mass. $F_{1}^{\gamma \gamma}$ is the proton's electromagnetic structure function and we also note that, in a similar manner to Eq. (7.1), $x_{\pi}$ and $W_{\pi}$ refer to the pion production threshold.

There has recently been considerable interest in precisely determining the $Q^{2}$-dependence of $\alpha$ and $\beta$ both experimentally [162, 168, 169], and theoretically $[170,171]$. Liang et al. used $e-p$ scattering data from the JLab E94-110 experiment [113] to measure the second moments of the $F_{1}^{\gamma \gamma}$ structure function. From these measurements they extracted values for the generalised Baldin sum rule using Eq. (7.2). The sum $\alpha\left(Q^{2}\right)+\beta\left(Q^{2}\right)$ was obtained for a number of kinematic points over the range $0.3<Q^{2}<4 \mathrm{GeV}^{2}$. An additional feature of their analysis was the separation of the structure function integral into a resonance $\left(W^{2}<4 \mathrm{GeV}^{2}\right)$ and a DIS $\left(W^{2}>4 \mathrm{GeV}^{2}\right)$ section. (For the DIS region the authors used the SLAC parametrisation of $R=F_{L} / 2 x F_{1}$ and $F_{2}$ from Ref. [172]).

Theoretically, Sibirtsev and Blunden made use of updated data [112] from the same E94-110 experiment to construct a parametrisation of the electromagnetic $F_{1}^{\gamma \gamma}$ and $F_{2}^{\gamma \gamma}$ structure functions [108, 170]. As discussed in earlier chapters, this parametrisation was used as the basis of the interference structure function inputs in the work on the $\gamma Z$ box contribution [108]. In Ref. [170], however, the electromagnetic $F_{1}^{\gamma \gamma}$ parametrisation was used in the Baldin integral to determine the sum of the polarisabilities. 

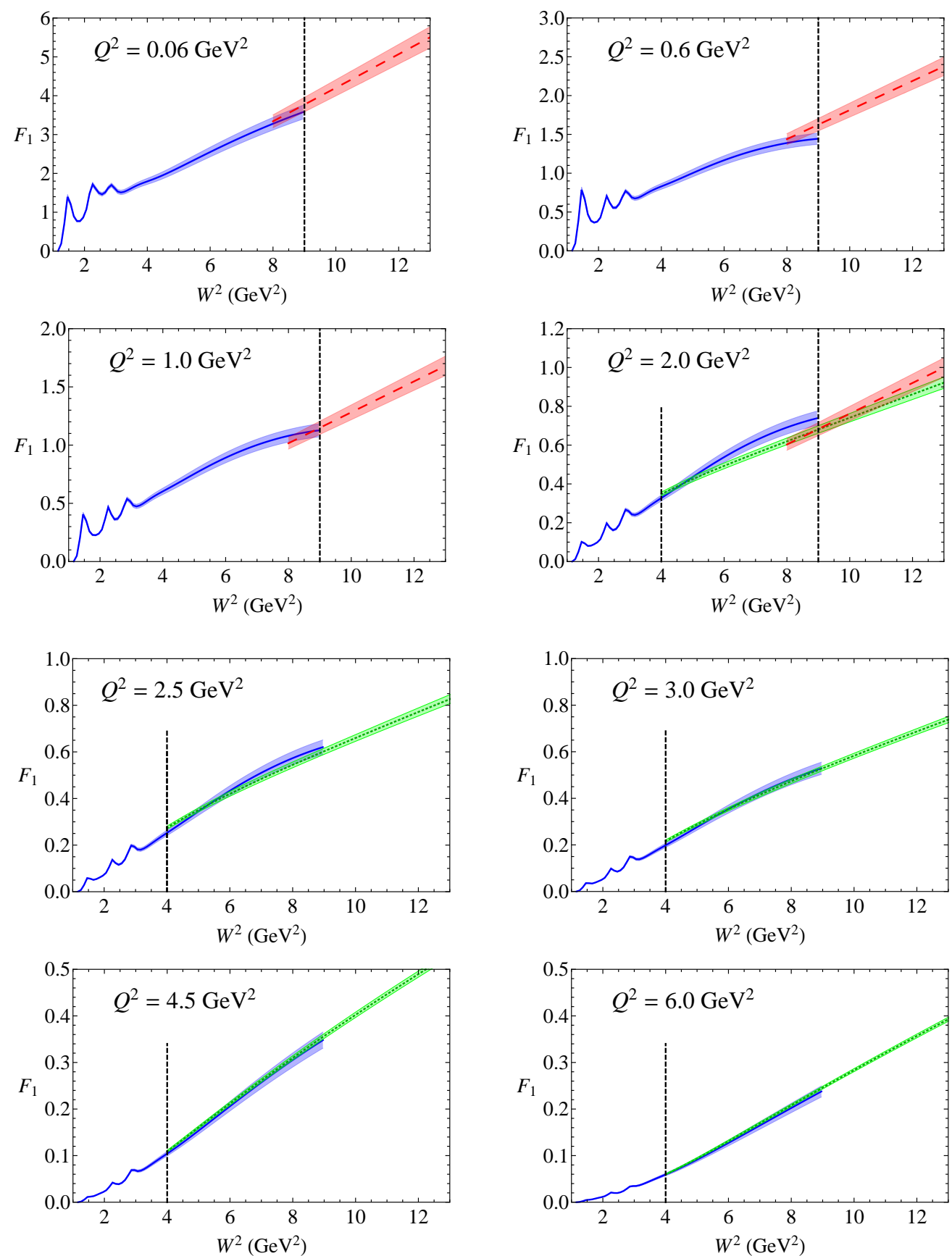

Figure 7.1: Proton $F_{1}$ structure function versus $W^{2}$ at fixed $Q^{2}=0.06,0.6$, 1.0 and $1.5 \mathrm{GeV}^{2}$ for the $\mathrm{CB}$ fit [121] at low $W^{2}$ (blue solid), VMD+Regge parametrisation [134] at high $W^{2}$ (red dashed) and ABM11 [141] (green dotted) parametrisations. The boundaries between these regions are indicated by the vertical dashed lines at $W^{2}=4$ and $9 \mathrm{GeV}^{2}$. 
The results of Sibirtsev and Blunden provide valuable information on the low- $Q^{2}$ dependence of $\alpha\left(Q^{2}\right)+\beta\left(Q^{2}\right)$, with values determined as low as $Q^{2}=$ $0.225 \mathrm{GeV}^{2}$ (cf. Fig. 4 of Ref. [170]). Additionally, they were able to examine the purely resonance contribution to the generalised Baldin integral, finding that for $Q^{2}<1 \mathrm{GeV}^{2}$ the resonances dominate [170]. Since the parameters of their model were fit only to $Q^{2}$ data above $0.225 \mathrm{GeV}^{2}$, however, Sibirtsev and Blunden were unable to provide an estimate of the static polarisablities. In fact, an extrapolation of their results would see them significantly overestimate $\alpha+\beta$-see Fig. 7.2.

In the next section we improve upon the work of Sibirtsev and Blunden, exploiting the AJM parametrisation of the $F_{1}^{\gamma \gamma}$ electromagnetic structure function to evaluate the generalised Baldin sum rule. Since this parametrisation is consistent down to $Q^{2}=0.06 \mathrm{GeV}^{2}$ we will be able to show the momentum transfer dependence of the electric and magnetic polarisabilities to much lower $Q^{2}$ than that in Ref. [170]. Furthermore, extrapolating our results to the photoproduction point results in a good estimate of $\alpha+\beta$. Before proceeding to the evaluation of the sum rule, however, the consistency of the structure functions at the boundaries between the different regions must first be checked. To do so we plot $F_{1}^{\gamma \gamma}\left(W^{2}, Q^{2}\right)$ as a function of $W^{2}$ for multiple values of $Q^{2}$ ranging from $Q^{2}=0.06$ to $6.0 \mathrm{GeV}^{2}$. Illustrated in Fig. 7.1, these plots make it clear that as for $F_{2}^{\gamma \gamma}$, the descriptions of the structure functions are in good agreement at the borders. As discussed in Chapter 5, for the (blue) ChristyBosted structure functions we assign a $5 \%$ error. This is based on the fact that more than half the data points differed from their fit by $3 \%$ or less, and almost all by less than 5\% [121]. For the (green) PDF structure functions the errors are those given by Alekin et al. in Ref. [141], while for the Region II structure functions, comparing the VMD+Regge model with data shows that assigning a $5 \%$ error on this model should suffice to account for any variation with experimental values.

\section{2 $Q^{2}$ dependence of $\alpha$ and $\beta$}

Since we would like to determine the $Q^{2}$-evolution of the electromagnetic polarisabilities all the way to the real photon point, the behaviour of the $F_{1}^{\gamma \gamma}$ structure function as $Q^{2} \rightarrow 0$ is particularly important. Christy and Bosted's parametrisation includes data down to $Q^{2}=0.06 \mathrm{GeV}^{2}$ as well at $Q^{2}=0$ and shows excellent agreement with the empirical data in the regions where data exists. However, no data exists for $Q^{2}$ between $0-0.06 \mathrm{GeV}^{2}$ and the fit fails to smoothly match onto the real photon point. Difficulties arise in the second resonance region where, although the overall magnitude of the transverse resonance is consistent with data, the individual transition form factors of the fit are not (cf. Table III of Ref. [121]) and result in a discontinuity in the cross sections between the lowest finite momentum transfer and the photoproduction point. In order to surmount this is- 


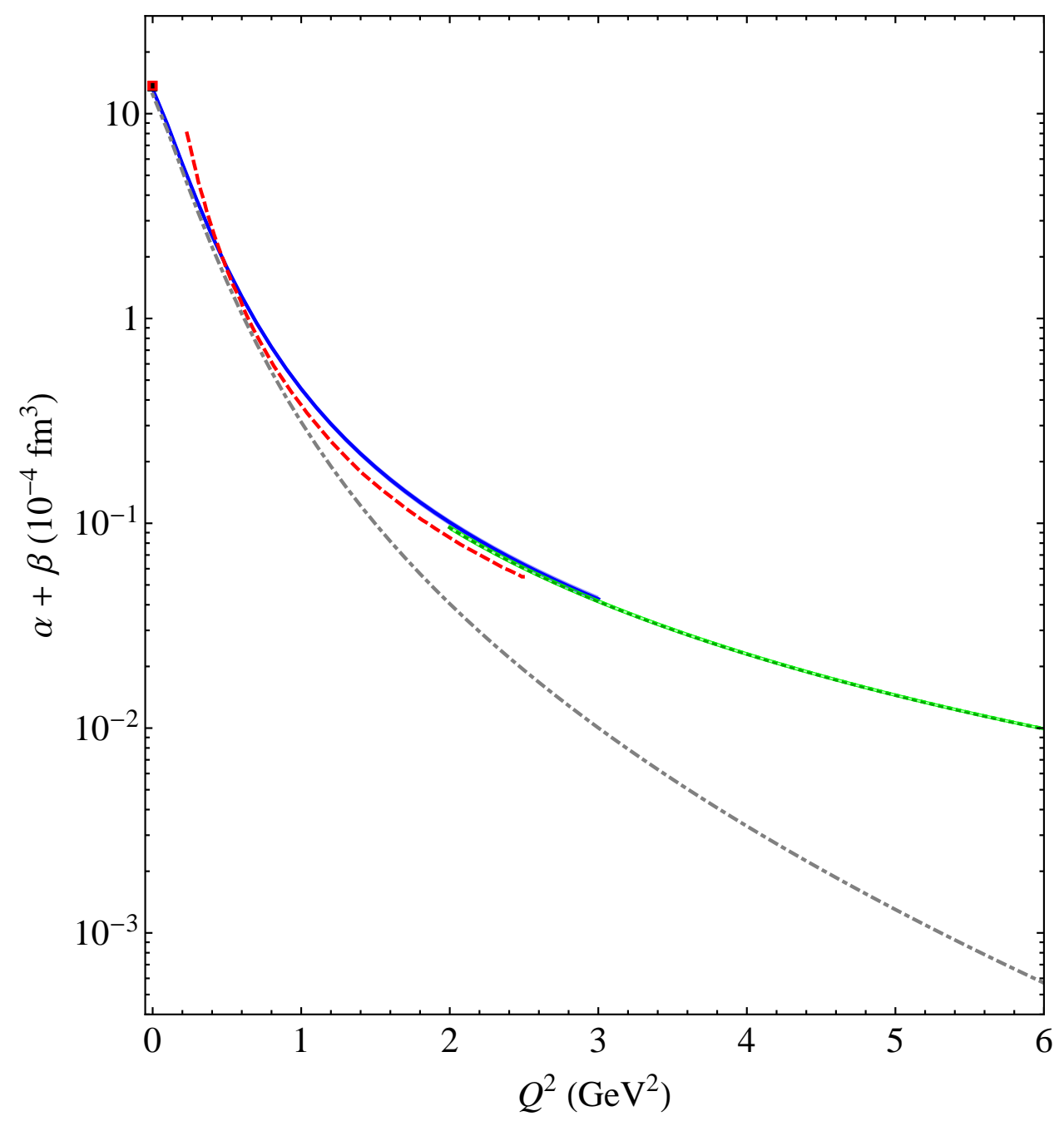

Figure 7.2: The sum of the electric and magnetic polarisabilities as a function of $Q^{2}$. The blue line (with errors) represents our calculation for $Q^{2} \leq 3.0 \mathrm{GeV}^{2}$, the green dotted line for $Q^{2}>2.0 \mathrm{GeV}^{2}$, whilst the red dashed line is the earlier calculation of Ref. [170]. The grey, dot-dashed line denotes the contributions to the polarisabilities from the $W^{2}<4.0 \mathrm{GeV}^{2}$ region. On this scale the values of $\alpha+\beta$ at the real photon point overlay each other and are given by the black triangle [173] and red square [174]. 


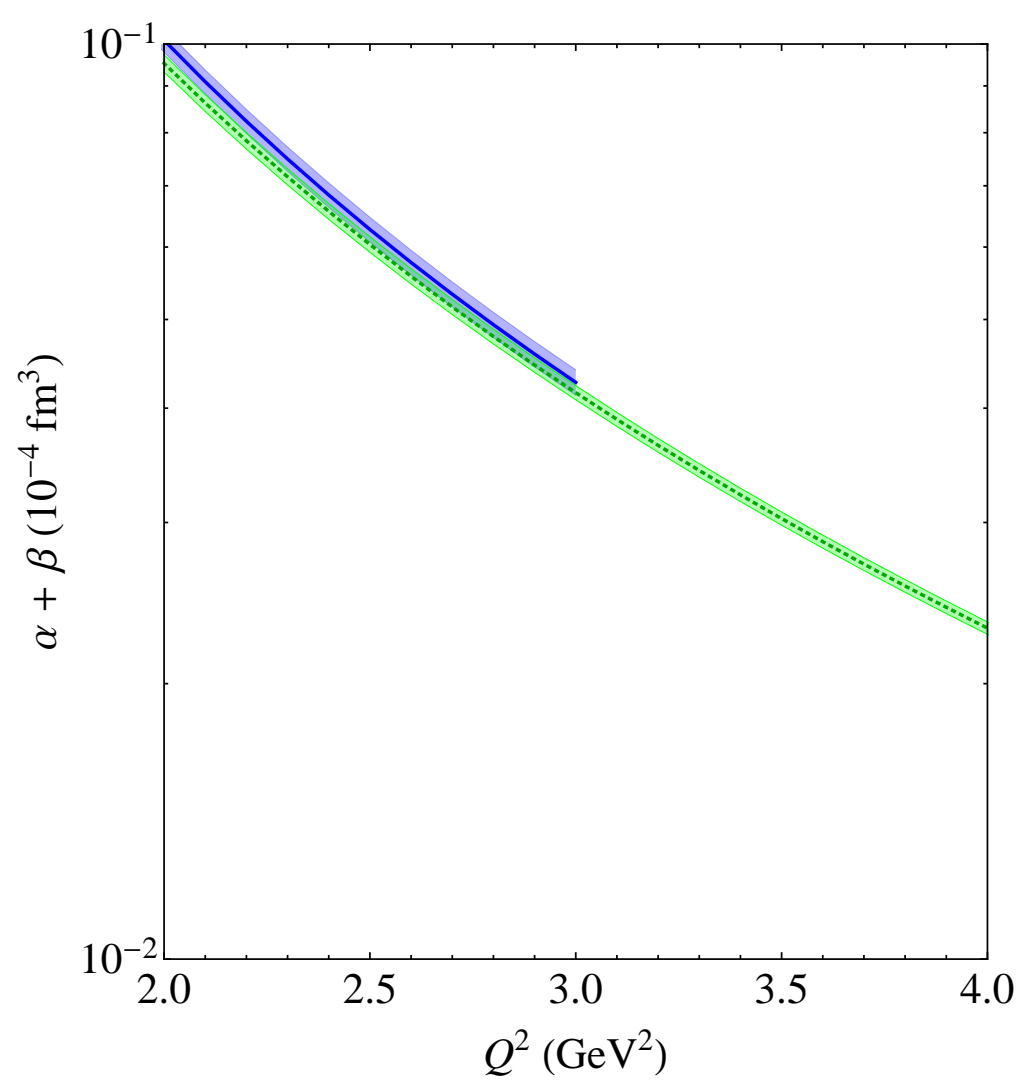

Figure 7.3: As in Fig. 7.2 but with the boundary region $2 \leq Q^{2} \leq 4 \mathrm{GeV}^{2}$ scaled up. The red dashed line of Sibirtsev and Blunden has been dropped since here we are solely interested in the overlap of the (blue) $Q^{2} \leq 3.0 \mathrm{GeV}^{2}$ and (green) $Q^{2}>2.0 \mathrm{GeV}^{2}$ regions.

sue, we use CB's $F_{1}^{\gamma \gamma}$ structure functions to evaluate $\alpha\left(Q^{2}\right)+\beta\left(Q^{2}\right)$ down to $Q^{2}=0.06 \mathrm{GeV}^{2}$, before extrapolating to $Q^{2}=0 \mathrm{GeV}^{2}$. We estimate the uncertainty arising from this extrapolation by using several different ranges to constrain the extension to zero momentum transfer.

The results of our analysis are illustrated in Fig. 7.2. We evaluate the Baldin integral over the range $0.06 \leq Q^{2} \leq 6 \mathrm{GeV}^{2}$ and include in the plot the calculation of Ref. [170] for comparison. In Fig. 7.3 we show a magnified version of the boundary region, displaying good overlap between the two. Note that although Fig. 7.2 shows the $Q^{2}$-dependence up to $6 \mathrm{GeV}^{2}$, the only limitation on the range is the validity of the parton distributions. One could in principle show results up to LHC energies.

From Fig. 7.2 we see that the $Q^{2}$-evolution of the AJM parametrisation differs significantly from that of Ref. [170]. Most importantly, as $Q^{2}$ approaches zero, $\alpha\left(Q^{2}\right)+\beta\left(Q^{2}\right)$ converges towards the static polarisabilities, where as an extrapolation of Sibirtsev and Blunden's results would considerably over estimate this value. The difference between these two results may be understood by the fact that CB's fit of the cross sections includes data at much lower values of $Q^{2}$. From the grey dot-dashed curve in Fig. 7.2 and the blue dashed 


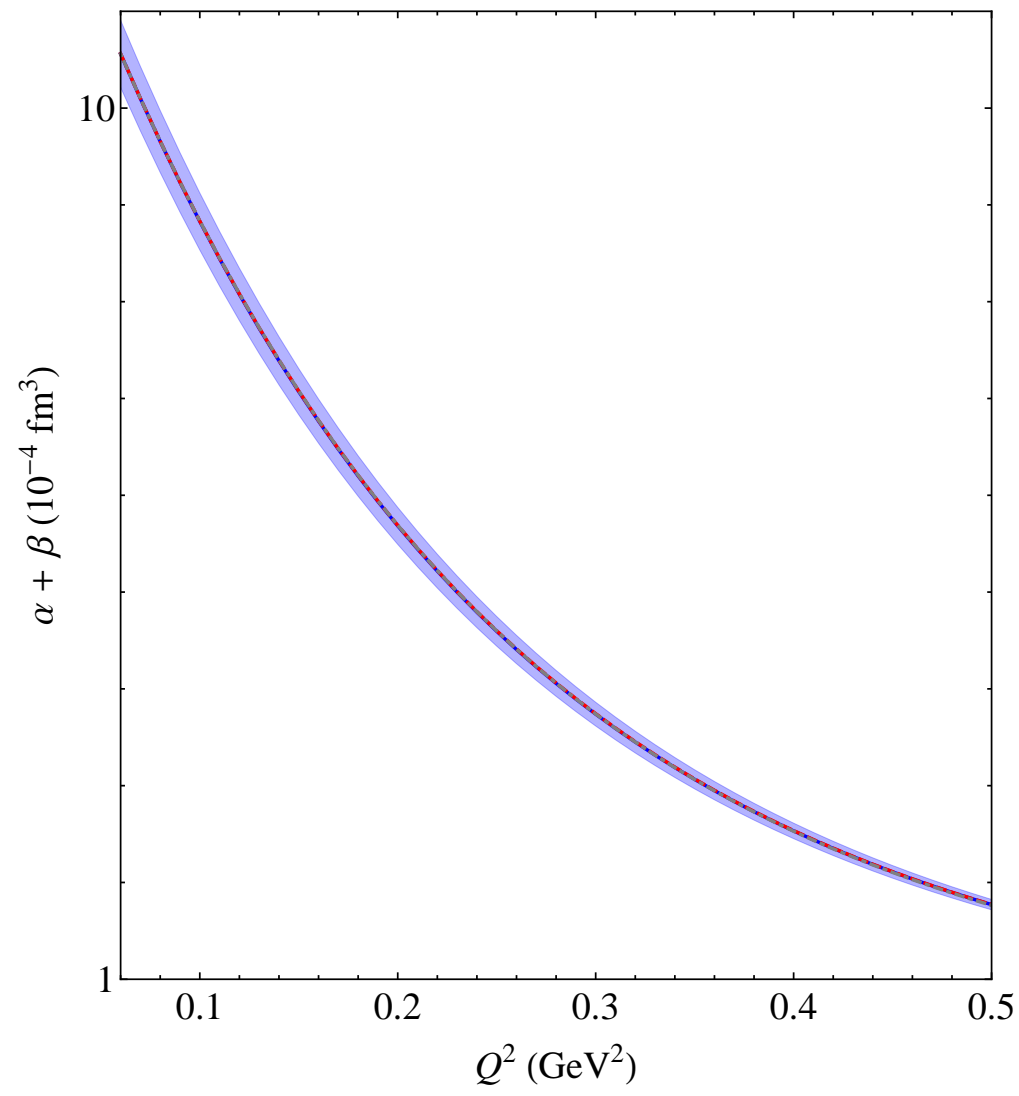

Figure 7.4: The results for the electromagnetic polarisabilities using $W^{2}=4$ (gray dot-dashed), 6 (red dashed) and $9 \mathrm{GeV}^{2}$ (blue solid) as the boundary between Regions I and II. All three curves sit directly above each other.

line in Fig. 4 of Ref. [170], it is, however, seen that both models agree that the resonance contribution dominates the Baldin integral. We also note that setting the boundary between Regions I and II at different $W^{2}$ has negligible effect on the final value. This is seen diagrammatically in Fig. 7.4.

For $Q^{2}$ below $0.06 \mathrm{GeV}^{2}$, we fitted the results of the generalised Baldin sum rule to the inverse of a polynomial over four different ranges of $Q^{2}$ :

$$
\begin{aligned}
& \text { I: } 0.04<Q^{2}<0.10 \mathrm{GeV}^{2} ; \\
& \text { II: } 0.04<Q^{2}<0.12 \mathrm{GeV}^{2} ; \\
& \text { III: } 0.06<Q^{2}<0.10 \mathrm{GeV}^{2} ; \\
& \text { IV: } 0.06<Q^{2}<0.12 \mathrm{GeV}^{2} \text {. }
\end{aligned}
$$

In order to obtain $\alpha+\beta$, each of these fits were extrapolated to $Q^{2}=0 \mathrm{GeV}^{2}$. The extrapolations are illustrated in Fig. 7.5 and show minimal variation over the various $Q^{2}$ ranges.

At the photoproduction point,

$$
\alpha+\beta=(13.7 \pm 0.7) \times 10^{-4} \mathrm{fm}^{3},
$$




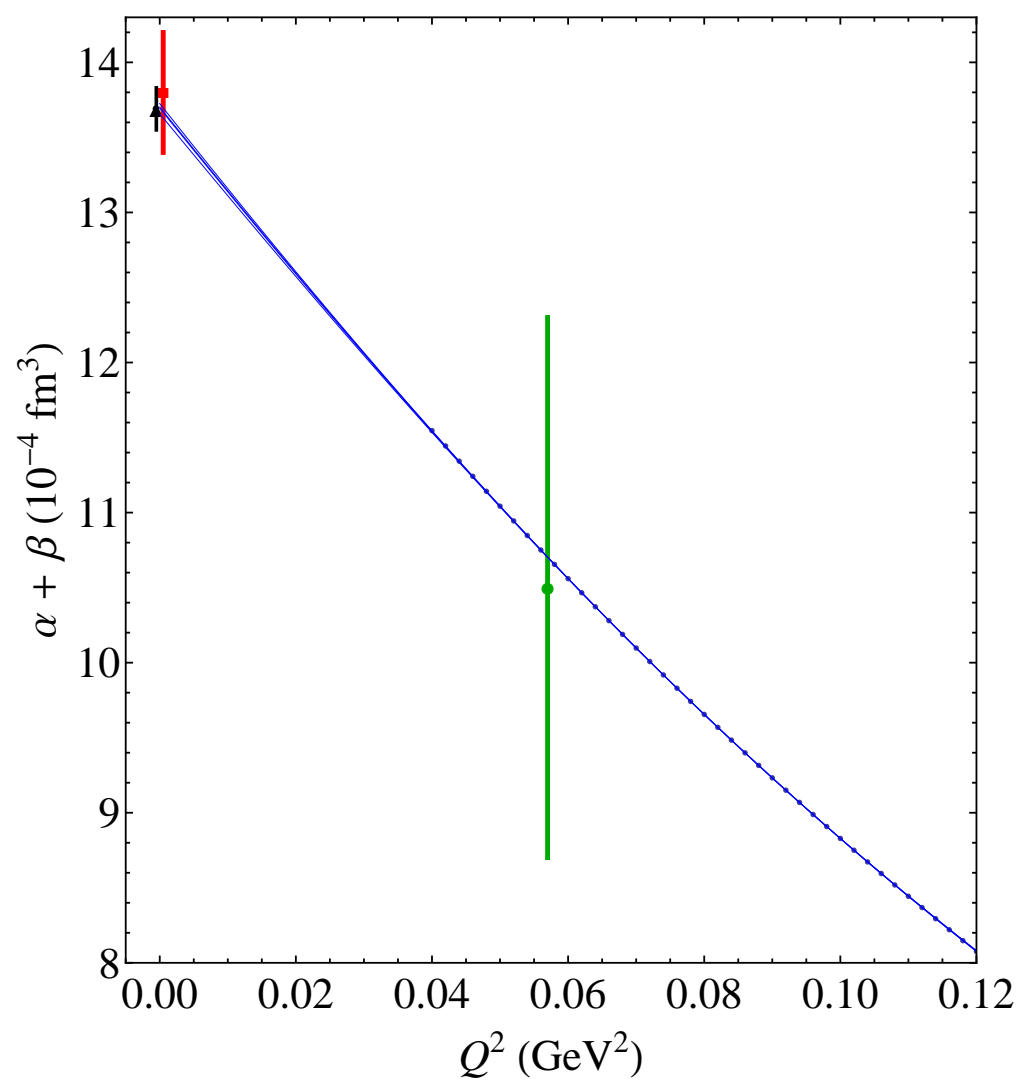

Figure 7.5: In this plot, the individual points are values extracted using the AJM parametrisation, whilst the blue lines are fits to points over four distinct ranges in $Q^{2}$. The values of $\alpha+\beta$ at $Q^{2}=0 \mathrm{GeV}^{2}$ have been offset slightly and are given by the black triangle [173] and red square [174].

where we use the fit over the $Q^{2}$ ranges given in III for the final results. The uncertainty \pm 0.7 on $\alpha+\beta$ comes from the $5 \%$ error assigned to the $\mathrm{CB} F_{1}^{\gamma \gamma}$ structure function. Our determination is in excellent agreement with the earlier evaluation of Babusci et al. [173], who give,

$$
\alpha+\beta=(13.69 \pm 0.14) \times 10^{-4} \mathrm{fm}^{3},
$$

and the more recent analysis,

$$
\alpha+\beta=(13.8 \pm 0.4) \times 10^{-4} \mathrm{fm}^{3},
$$

of Ref. [174]. Although both data points have been included in Figs. 7.5 and 7.2 , the latter is not only more recent, but stems from more conservative uncertainty estimates [161].

Finally, we may determine the 'radius' of the sum of the electric and magnetic polarisabilities, i.e.,

$$
\left\langle r^{2}\right\rangle=\left.\frac{-6}{H(0)} \frac{d H\left(Q^{2}\right)}{d Q^{2}}\right|_{Q^{2}=0},
$$


where, $H\left(Q^{2}\right)=\alpha\left(Q^{2}\right)+\beta\left(Q^{2}\right)$. Using the same fit which gave the $Q^{2}$ extrapolation, we found,

$$
\left\langle r^{2}\right\rangle_{\alpha+\beta}^{1 / 2}=0.98 \pm 0.05 \mathrm{fm},
$$

which agrees well with the heavy baryon chiral perturbation theory $(\mathrm{HB} \chi \mathrm{PT})$ value of $\sim 1.1 \mathrm{fm}$ given in Ref. [175].

The agreement of the AJM parametrisation with the earlier determinations of the static electromagnetic polarisabilities, the good convergence to $Q^{2}=0$ point and the compatibility with the $\mathrm{HB} \chi \mathrm{PT}$ value of the radius gives further evidence of the robustness of these structure functions.

Utilising a parametrisation of the electromagnetic $F_{1}^{\gamma \gamma}$ structure function consistent down to $Q^{2}=0.06 \mathrm{GeV}^{2}$, we have shown that the $Q^{2}$-dependence of the electromagnetic polarisabilities converges to the photoproduction point. While our results at the real photon point agree well with the work of Refs. [173, $174,175]$ the evolution of $\alpha\left(Q^{2}\right)+\beta\left(Q^{2}\right)$ differs substantially from Sibirtsev and Blunden. The variation we observe is accounted for by the fact that our parametrisation is fitted to much lower $Q^{2}$. On the other hand, the deviation at higher momentum transfer comes from the difference between their parametrisation and Christy-Bosted's.

Although the uncertainties on the sum of $\alpha$ and $\beta$ are similar to other determinations, it would be useful to reduce them still further. Additional data in the very low- $Q^{2}$ and low- $W^{2}$ region would remove the need for extrapolation and increase the precision. Given the importance of understanding the nature of the electromagnetic structure of the nucleon, there is a well motivated need for further experimental efforts. 



\section{Quark-hadron duality}

In spite of the apparent differences between the partonic and hadronic realisations of QCD, at low energies there are instances where the averaged cross sections coincide with those determined using the parton model. This behaviour is known as 'quark-hadron duality', and in this chapter we make use of the AJM model to study this phenomenon.

\subsection{Duality in the SM}

Quark-hadron duality in the Standard Model is the physical manifestation of the relationship between confinement and asymptotic freedom, and marks the transition between perturbative and nonperturbative QCD. Duality is observed in many different areas such as $e^{+} e^{-}$annihilation, semi-leptonic decays and electron-nucleon scattering. This latter field is what we are particularly interested in since it links the physics surrounding resonance production with scaling. Given that structure functions cover both of these regions, it is no surprise they are one of the tools used to study this subject.

Historically, duality was observed even before the formulation of QCD and its acceptance as the correct description of the strong interaction. Bloom and Gilman found that resonance structure functions at low invariant mass averaged to the scaling curve which accounted for the high- $W$ values [176, 177]. After QCD was invented, duality was expressed in terms of the operator product expansion [178, 179], and its violation was seen as a consequence of higher-twist operators which described long range physics [180]. The OPE, however, still failed to explain the physics of how the resonances transitioned into the scaling regime and it was not until the advent of recent low-energy precision measurements of structure functions that duality became an active area of research once more. An example of modern evidence for duality in the proton structure functions is illustrated in Fig. 8.1.

At large energies, duality between the partonic and hadronic descriptions may understandably be considered exact. What is perhaps not so expected, is the 'local' duality seen in the JLab precision data [181], where the averaged resonance and scaling structure functions appear to match even in the indi- 


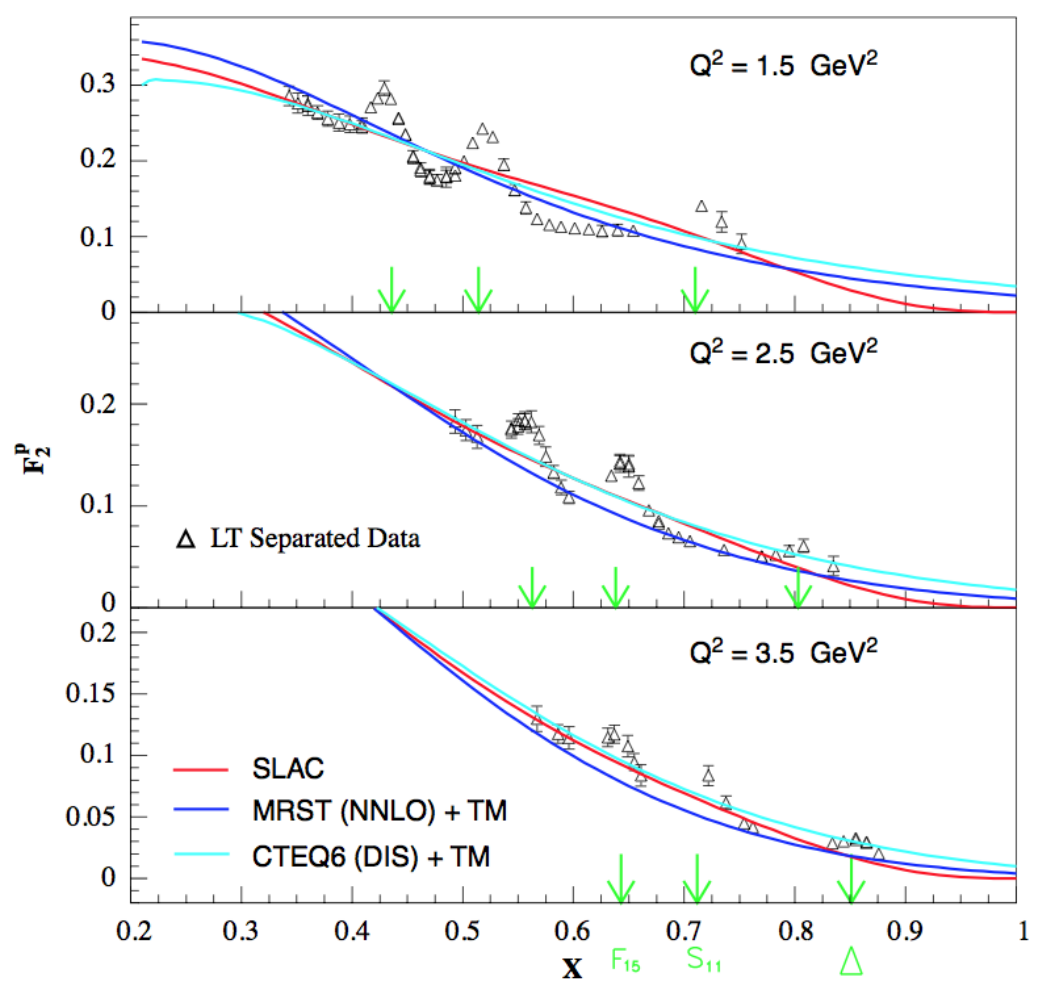

Figure 8.1: Example of duality in the $F_{2}^{p}$ structure function [180]. Target mass (TM) corrections are included in the MRST and CTEQ6 parametrisations of the PDFs and the data points come from the JLab E94-110 experiment $[113,182]$.

vidual resonance regions. This is particularly puzzling since at these low $Q^{2}$, the strong coupling constant is relatively large and therefore perturbation theory is no longer valid. Additionally, the degree to which duality holds and the kinematic range to which it applies is difficult to quantify. All these questions have played a large role in motivating the renewed efforts - both theoretically and experimentally - to study duality.

Recent work has looked at a number of aspects of duality including, its flavour, spin and nuclear dependence $[181,183,184,185,186]$. It has also been investigated in neutrino scattering experiments [151]. Our own interest in the topic is twofold: firstly, since in the AJM model we have a reliable description of both the $\gamma \gamma$ and $\gamma Z$ structure functions, we have the necessary tools to study and compare duality in both the electromagnetic and interference cases; secondly, duality arguments may also be used to further constrain the $\gamma Z$ structure functions. In electron-nucleon scattering, duality has been observed as low as $Q^{2}=1 \mathrm{GeV}^{2}[180]$. Should the $\gamma Z$ resonance structure functions in this region behave similarly, their averaged curves will be restricted to values close to the LT structure functions.

The rest of the chapter is arranged as follows: in the next section we begin by briefly describing the formalism surrounding the twist expansion of 
the moments of structure functions. The relationship between the Nachtmann moments which include target mass corrections (TMCs), and the CornwallNorton moments are also included in this section. After this we present results using the AJM model, before finally in Section 8.3, we discuss the implications for the $\square_{\gamma Z}^{V}$ corrections.

\subsection{Moments of structure functions}

The OPE $[187,188,189]$ is used to theoretically describe Bloom-Gilman duality in QCD. Intuitively the OPE provides a way of separating out the nonperturbative physics of parton correlation functions from the perturbative part of hard scattering. More precisely, it involves expanding the product of two operators with small light-cone separation into a sum of local operators [190]. Quantities most suited for study within the framework of the OPE analysis are the structure function moments.

The Cornwall-Norton moments, are defined as [191],

$$
\begin{aligned}
& M_{1}^{n}\left(Q^{2}\right)=\int_{0}^{1} d x x^{n-1} F_{1}\left(x, Q^{2}\right), \\
& M_{2}^{n}\left(Q^{2}\right)=\int_{0}^{1} d x x^{n-2} F_{2}\left(x, Q^{2}\right) .
\end{aligned}
$$

At $Q^{2}$ much greater than the QCD energy scale $\left(\Lambda_{Q C D}^{2}\right)$ these moments may be expanded in powers of $1 / Q^{2}[180]$. For example, the above $M_{2}^{n}$ moment becomes,

$$
M_{2}^{n}\left(Q^{2}\right)=\sum_{\tau=2,4 \ldots}^{\infty} \frac{A_{\tau}^{n}\left(\alpha_{s}\left(Q^{2}\right)\right)}{Q^{\tau-2}}, \quad n=2,4,6 \ldots
$$

where the twist $\tau$ is defined as the difference between the mass dimension and the spin of the operator. The coefficients $A_{\tau}^{n}$ are matrix elements of quark and gluon operators [180]. The higher-twist (HT) terms $(\tau>2)$ contribute at smaller $Q^{2}$, whilst at larger $Q^{2}$ the leading-twist terms dominate. In Fig 8.2 we give examples of LT and HT diagrams which contribute to the structure functions.

Incorporating target mass corrections, the Nachtmann $\mu_{2}^{n}$ moment may be written $[188,192]$,

$$
\mu_{2}^{n}\left(Q^{2}\right)=\int_{0}^{1} d x \frac{\xi^{n+1}}{x^{3}}\left\{\frac{3+3(n+1) r+n(n+2) r^{2}}{(n+2)(n+3)}\right\} F_{2}\left(x, Q^{2}\right)
$$

where,

$$
\xi=\frac{2 x}{1+\sqrt{1+4 M^{2} x^{2} / Q^{2}}}
$$



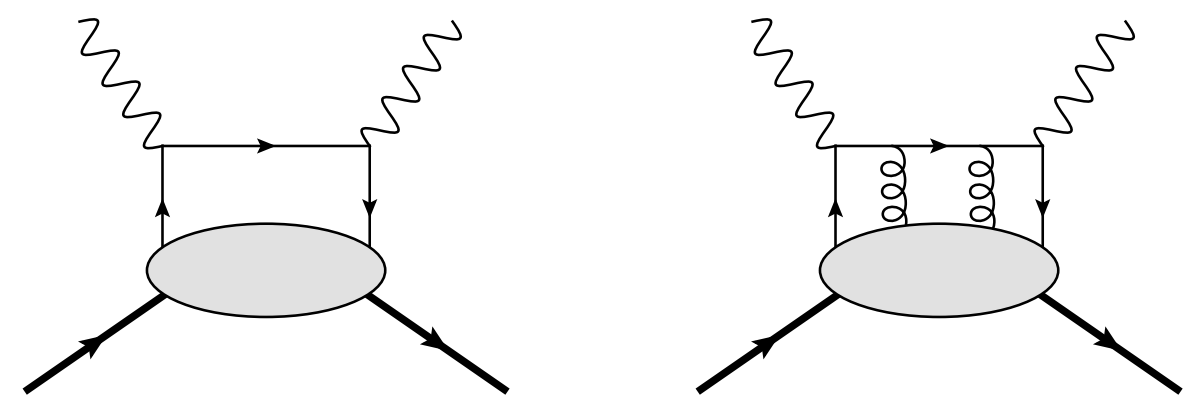

Figure 8.2: Leading- (left) and higher- (right) twist contributions to structure functions

is the new scaling variable, $r=\sqrt{1+4 M^{2} x^{2} / Q^{2}}$ and $M$ (with no numerical subscript) is the mass of the nucleon. The two descriptions are related via [193],

$$
\begin{aligned}
\mu_{2}^{n}\left(Q^{2}\right)= & M_{2}^{n}\left(Q^{2}\right)-\frac{n(n-1)}{n+2} \frac{M^{2}}{Q^{2}} M_{2}^{n+2}\left(Q^{2}\right) \\
& +\frac{n\left(n^{2}-1\right)}{2(n+3)} \frac{M^{4}}{Q^{4}} M_{2}^{n+4}\left(Q^{2}\right)-\frac{n\left(n^{2}-1\right)}{6} \frac{M^{6}}{Q^{6}} M_{2}^{n+6} \ldots
\end{aligned}
$$

or inversely [193],

$$
\begin{aligned}
M_{2}^{n}\left(Q^{2}\right)=\mu_{2}^{n}\left(Q^{2}\right) & +\frac{n(n-1)}{n+2} \frac{M^{2}}{Q^{2}} \mu_{2}^{n+2}\left(Q^{2}\right) \\
+ & \frac{n\left(n^{2}-1\right)(n+2)}{2(n+3)(n+4)} \frac{M^{4}}{Q^{4}} \mu_{2}^{n+4}\left(Q^{2}\right) \\
& +\frac{n\left(n^{2}-1\right)(n+2)(n+3)}{6(n+5)(n+6)} \frac{M^{6}}{Q^{6}} \mu_{2}^{n+6} \ldots
\end{aligned}
$$

Furthermore, as $Q^{2} \rightarrow \infty$, the two definitions of the moments coincide.

\section{Electromagnetic moments}

Using the AJM electromagnetic parametrisations we determine the moments of the nucleon's $F_{2}^{\gamma \gamma}$ structure function. Since the integral over $x\left(W^{2}\right)$ contains the elastic piece, the elastic structure functions must also be included [194],

$$
\begin{aligned}
& W_{1}\left(\nu, Q^{2}\right)=\frac{Q^{2}}{4 M^{2}}\left[G_{M}^{\gamma p}\left(Q^{2}\right)\right]^{2} \delta\left(\nu-\frac{Q^{2}}{2 M}\right) \\
& W_{2}\left(\nu, Q^{2}\right)=\frac{\left[G_{E}^{\gamma p}\left(Q^{2}\right)\right]^{2}+\frac{Q^{2}}{4 M^{2}}\left[G_{M}^{\gamma p}\left(Q^{2}\right)\right]^{2}}{1+\frac{Q^{2}}{4 M^{2}}} \delta\left(\nu-\frac{Q^{2}}{2 M}\right)
\end{aligned}
$$

where $G_{E}^{\gamma p}\left(Q^{2}\right)$ and $G_{M}^{\gamma p}\left(Q^{2}\right)$ are the electric and magnetic form factors of the nucleon, and

$$
\begin{aligned}
& F_{1}\left(x, Q^{2}\right)=M W_{1}\left(\nu, Q^{2}\right) \\
& F_{2}\left(x, Q^{2}\right)=\nu W_{2}\left(\nu, Q^{2}\right) .
\end{aligned}
$$


For the form factors, we use the standard Kelly parametrisation [195] and assign a conservative 5\% uncertainty to these elastic structure functions similar to previous chapters.

The proton and neutron second moments are shown over the range $1 \leq$ $Q^{2} \leq 9 \mathrm{GeV}^{2}$ in Fig. 8.3. ${ }^{1}$ Since QCD radiative corrections result in the LT structure functions evolving logarithmically, we plot the moments against $\log Q^{2}$. Also note, that as mentioned in Chapter 7 the larger uncertainty in the neutron plots are a result of the difficulty in extracting the neutron structure functions from deuteron data.

In determining the $\mu_{2}^{n}$ Nachtmann moments, the CB parametrisation is utilised in the range $\left(M_{N}+m_{\pi}\right)^{2} \leq W^{2} \leq 6 \mathrm{GeV}^{2}$, where $M_{N}$ is the mass of the proton $(N=p)$ or neutron $(N=n)$. For $W^{2}>6 \mathrm{GeV}^{2}$, we use Alekhin's full PDF structure functions. ${ }^{2}$ The term 'full' signifies that the structure functions include leading twists, TMCs, and higher-twist contributions in its parametrisation. (Note that here we use the ABM11 structure functions all the way to $1 \mathrm{GeV}^{2}$ as opposed to $2.5 \mathrm{GeV}^{2}$ for the $\square_{\gamma Z}^{V}$ analysis.) Combining these two parametrisations effectively gives the total structure functions and is denoted in Fig. 8.3 by the solid black line. The LT curve (red dashed line) also uses Alekhin's PDF structure functions, however only the LT contribution is included. For the LT structure functions, the Cornwall-Norton moments must be used instead of the Nachtmann moments. Furthermore, the PDFs are used over the entire kinematic region of the integral; although, since the elastic piece is purely HT, the LT moment does not include contributions from the form factors.

The results for the proton $\mu_{2}^{2}(p)$ moment - for which we know the structure functions most accurately - show remarkable agreement between the total structure function moment and the LT contribution. Although there is larger variation between the total and LT moments for the neutron, the LT curve remains within the uncertainties of the total moment and there is still a very strong correspondence between the two. (We assigned a $10 \%$ uncertainty to the neutron structure functions following Ref. [122].) The HT contributions can be found by taking the difference between the $\mu_{2}^{2}(N)$ moment given by the total structure function and that given by the LT expressions. In Fig. 8.4 we plot the magnitude of the higher-twist contributions relative to the total structure functions for the proton and neutron moments. Although the central curves for the proton's HT terms are predominantly below zero, taking into account uncertainties, the HT contributions are consistent with zero over the entire range of $Q^{2}$. While the neutron's higher twists are always positive, the large errors mean that these contributions are also consistent with zero.

Given that there is nothing in the physics of the interference cross sections to suggest that the $\gamma Z$ moments will be any different, we also expect the

\footnotetext{
${ }^{1} \mathrm{As}$ it is difficult to ascertain the reliability of the LT structure functions below $1 \mathrm{GeV}^{2}$ we limit the comparison to the range given.

${ }^{2} \mathrm{~A}$ comparison of the moments using different values for the $W^{2}$ boundary showed negligible variation over the range of $Q^{2}$ considered.
} 

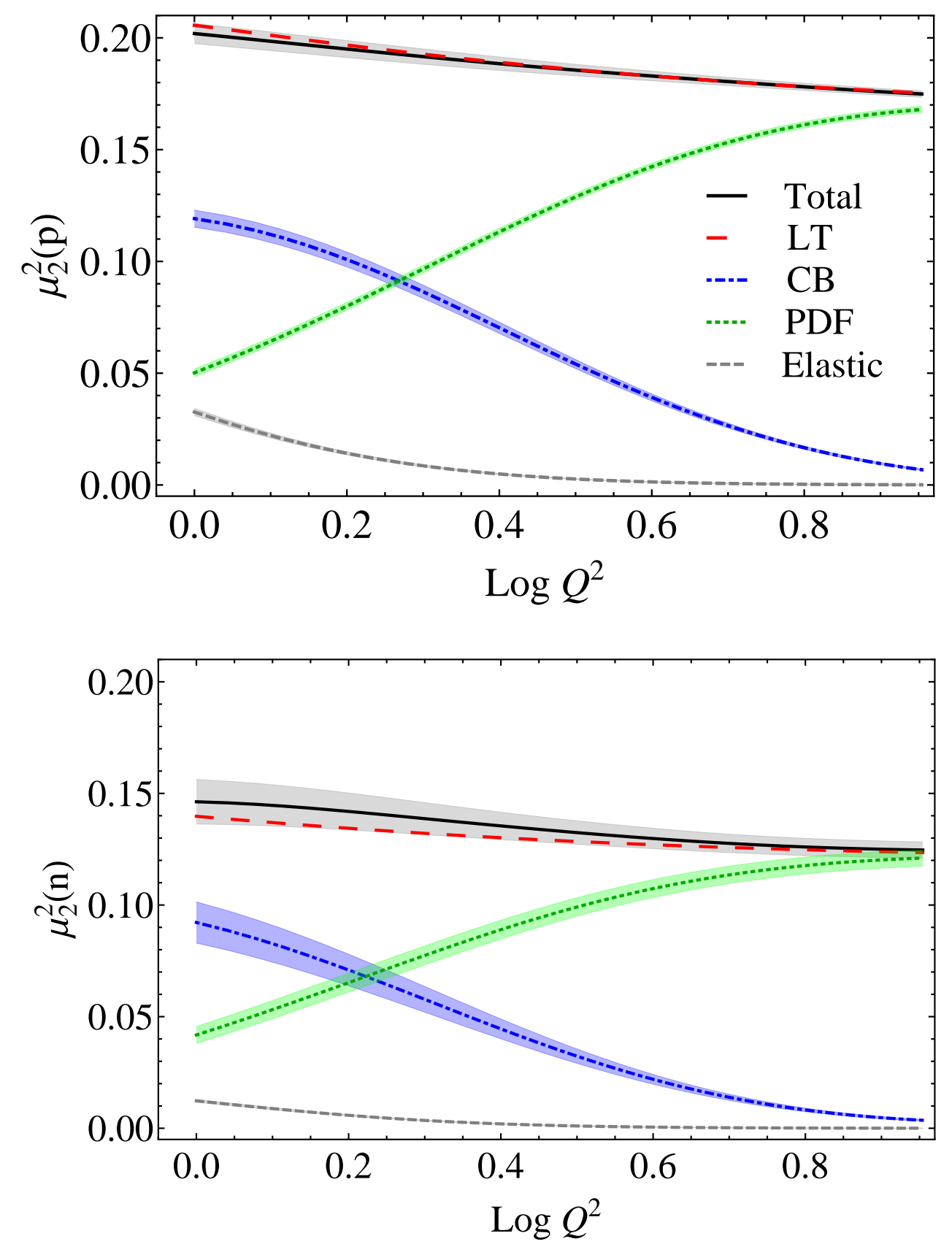

Figure 8.3: $\mu_{2}^{2}(N)$ moments using the electromagnetic structure functions. The upper figure represents the proton Nachtmann moment, and the lower figure the neutron Nachtmann moment. 


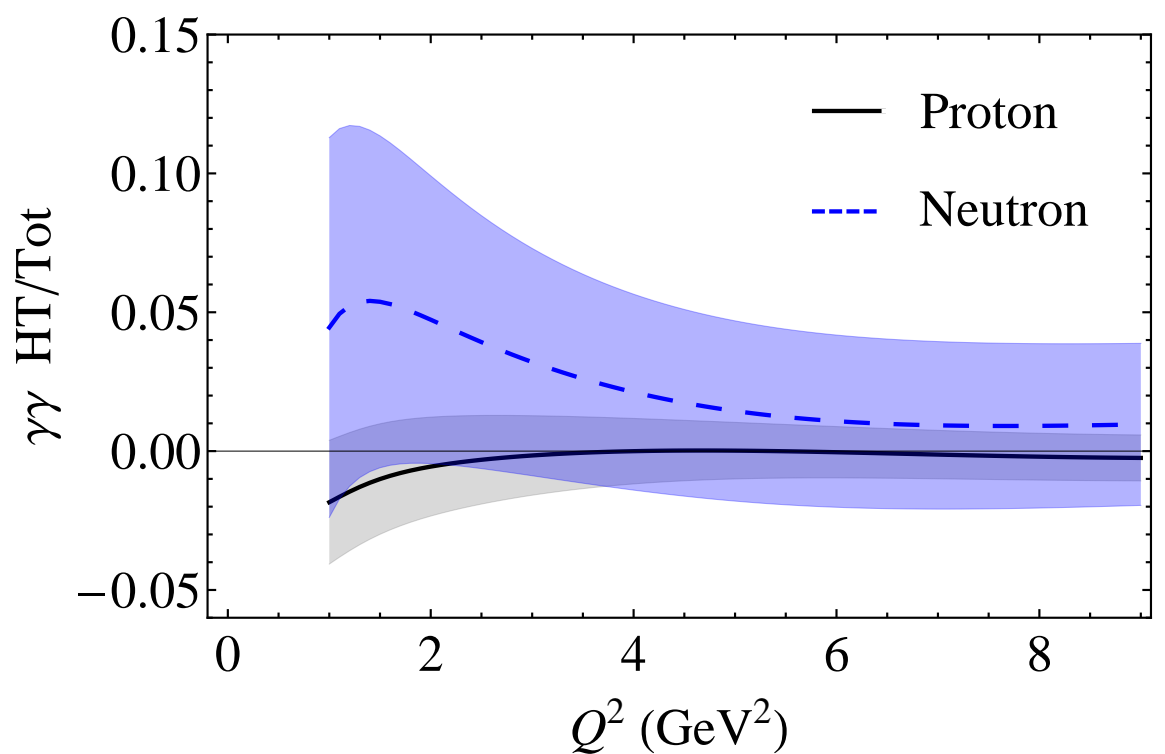

Figure 8.4: Relative HT contributions to the electromagnetic $\mu_{2}^{2}(N)$ moments.

HT contributions there to be small. This means that the averaged total $\gamma Z$ structure functions should be very similar to the averaged leading-twist structure functions - at least to $Q^{2}=1 \mathrm{GeV}^{2}$. In the next section we test this claim by calculating the moments determined using interference structure functions.

\section{$\gamma Z$ moments}

Using the AJM interference structure functions we may determine the proton and neutron's second moments. As before, we need the elastic forms of the (in this case $\gamma Z$ ) structure functions,

$$
\begin{aligned}
& F_{1}^{\gamma Z}\left(W^{2}, Q^{2}\right)=\frac{Q^{2}}{2} G_{M}^{\gamma p} G_{M}^{Z p} \delta\left(W^{2}-M^{2}\right) \\
& F_{2}^{\gamma Z}\left(W^{2}, Q^{2}\right)=\frac{Q^{2}}{1+\frac{Q^{2}}{4 M^{2}}}\left(G_{E}^{\gamma p} G_{E}^{Z p}+G_{M}^{\gamma p} G_{M}^{Z p}\right) \delta\left(W^{2}-M^{2}\right)
\end{aligned}
$$

where

$$
G_{E, M}^{Z p}=\left(1-4 \sin ^{2} \theta_{W}\right) G_{E, M}^{\gamma p}-G_{E, M}^{\gamma n}-G_{E, M}^{s},
$$

are the interference electric and magnetic form factors. In practice, the strangeness contribution, $G_{E, M}^{s}$, is neglected and charge symmetry is assumed exact. We assign $5 \%$ uncertainties to these elastic structure functions.

The calculation for the $\gamma Z$ moments follows in much the same manner as the electromagnetic ones. For the uncertainties, the proton's $\gamma Z$ structure functions are the same as those used in the $\square_{\gamma Z}^{V}$ calculation. The neutron's, however, were given a $15 \%$ error since this takes into account what is known about the deuteron $F_{i}^{\gamma Z}$ uncertainties, while still incorporating the difficulties 

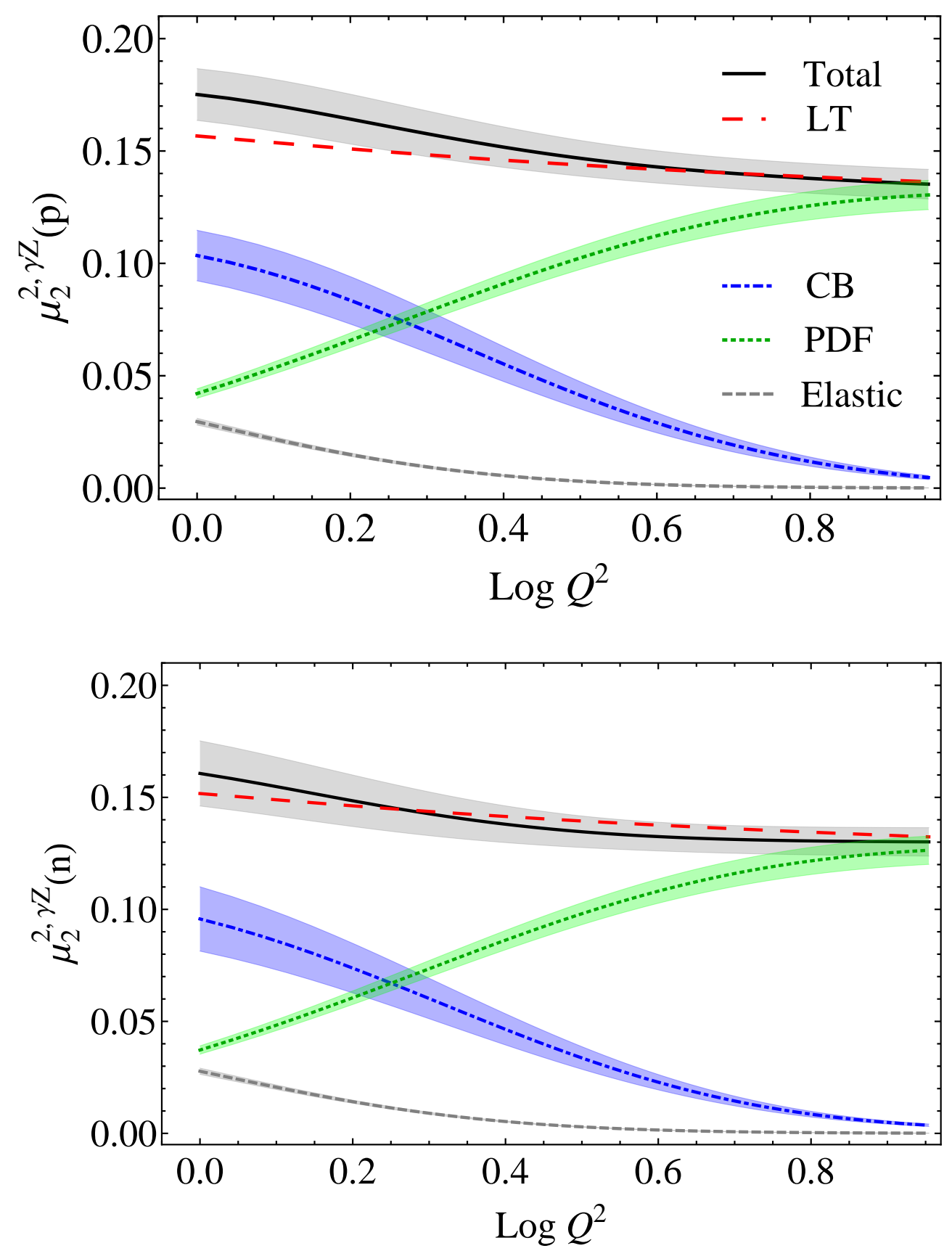

Figure 8.5: $\mu_{2}^{2}(N)$ moment using the $\gamma Z$ structure functions. The upper figure represents the proton Nachtmann moment, and the lower figure the neutron Nachtmann moment. 


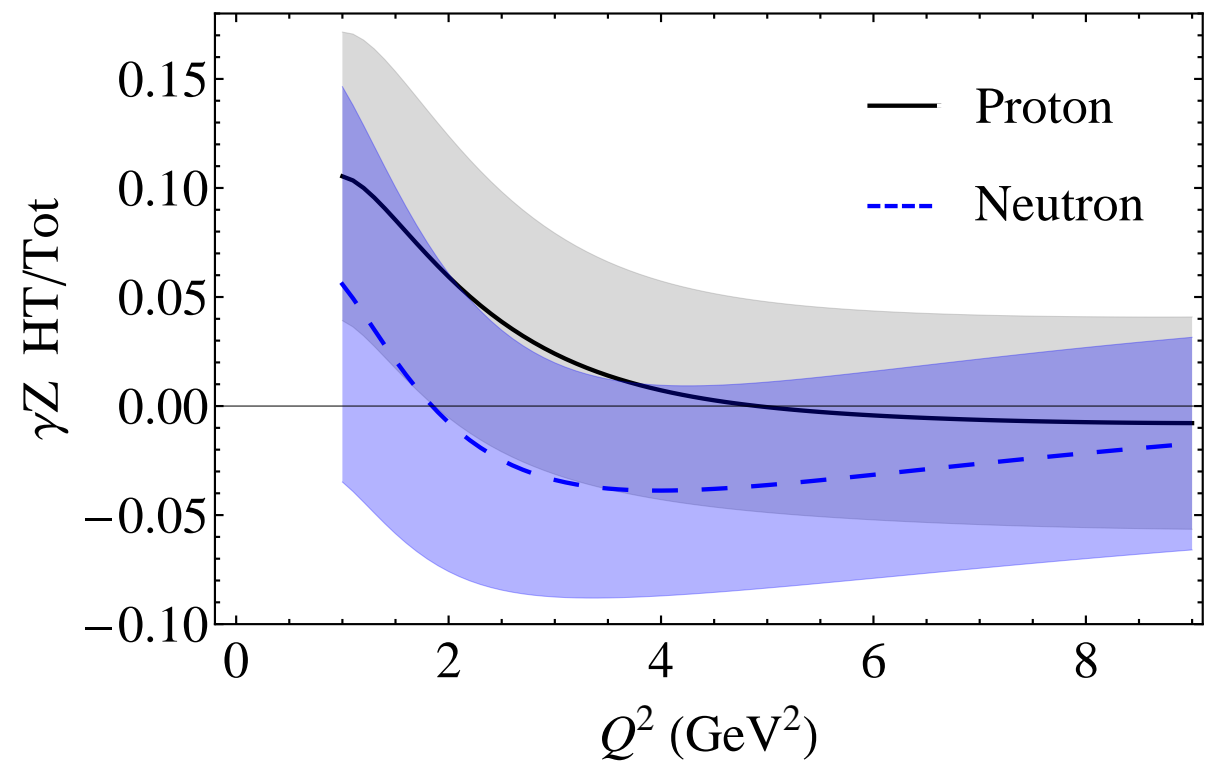

Figure 8.6: Relative HT contributions to the interference $\mu_{2}^{2}(N)$ moments.

in determining the neutron cross sections from them. In Fig. 8.5 we show the resulting $\gamma Z$ moments, while Fig. 8.6 gives the relative HT contributions.

As in the $\gamma \gamma$ structure function moments, the results for $\mu_{2}^{2}(N)$ using the interference structure functions show that HTs contribute only a small amount to the total structure functions. This confirms what was concluded from the electromagnetic moments. While the relative contributions from the higher twists are larger in the $\gamma Z$ case, the uncertainties are also larger and the results are consistent with those in Fig. 8.4.

\subsection{Implications for the $\square_{\gamma Z}^{V}$ correction}

In order to determine the effects of the moment results on the $\square_{\gamma Z}$ calculation, the hadronic vector (and for completeness the axial-vector) correction from the DIS region may be expanded in terms of moments [101],

$$
\begin{gathered}
\square_{\gamma Z}^{V}=\frac{2 M E}{\pi} \int_{Q_{0}^{2}}^{\infty} d Q^{2} \frac{\alpha\left(Q^{2}\right)}{Q^{4}\left(1+Q^{2} / M_{Z}^{2}\right)}\left[M_{2}^{2}\left(Q^{2}\right)\right. \\
+\frac{2}{3} M_{1}^{2}\left(Q^{2}\right)+\frac{2 M^{2}}{3 Q^{4}}\left(E^{2}-Q^{2}\right) M_{2}^{4}\left(Q^{2}\right) \\
\left.\quad+\frac{2 M^{2}}{5 Q^{4}}\left(4 E^{2}-5 Q^{2}\right) M_{1}^{4}\left(Q^{2}\right)+\cdots\right], \\
\square_{\gamma Z}^{A}=\frac{3}{2 \pi} \int_{Q_{0}^{2}}^{\infty} d Q^{2} \frac{v_{e}\left(Q^{2}\right) \alpha\left(Q^{2}\right)}{Q^{2}\left(1+Q^{2} / M_{Z}^{2}\right)}\left[M_{3}^{1}\left(Q^{2}\right)\right. \\
\left.+\frac{2 M^{2}}{9 Q^{4}}\left(5 E^{2}-3 Q^{2}\right) M_{3}^{3}\left(Q^{2}\right)+\cdots\right] .
\end{gathered}
$$


There are two conclusions that we may draw. The first, is that duality between the scaling region and the resonance structure functions to a large degree holds to $Q^{2}$ at least as low as $1 \mathrm{GeV}^{2}$. This means that the DIS region structure functions may be used to a lower $Q^{2}$ than was done in the AJM model (where Region III only began at $Q^{2}=2.5 \mathrm{GeV}^{2}$ ) and therefore the $M_{2}^{2}$ moment, which already dominates, will contribute an even larger amount. This would decrease the uncertainty in the final box correction since the PDFs are much better understood. (Even though the parton distributions do not have any resonance structure in them, the fact that the $\square_{\gamma Z}^{V}$ correction involves an integral meansbecause duality holds - that the PDFs will still give a good approximation to the final result.)

The second conclusion that may be drawn relates to the continuum piece in the transformation of the $\gamma \gamma$ cross section background to the $\gamma Z$ case. Again, looking at Figs. 8.3 and 8.5, since the electromagnetic LT and total structure function moments agree so well, it means that there is only a minimal amount of contribution from HTs. Since the total structure function is then constrained to agree with the LT within certain limits, and since the LT structure functions are precisely known, the total structure functions are constrained to values within a certain range from the LT structure functions. This means that the background contribution - and thus the continuum parameter which modifies the background - is also constrained to within a certain limit of the LT structure functions. As the results for the $\gamma Z$ moments themselves show good agreement with the LT, this confirms the conclusions drawn from the electromagnetic moment.

It is thus clear from the results in this chapter that the uncertainties on the $\square_{\gamma Z}^{V}$ correction given by the AJM, PDF constrained model are well soundly based. The results for the moments confirm our earlier argument, based on PDF constraints and deuteron PV asymmetry results, that the uncertainties for the $\square_{\gamma Z}^{V}$ correction are severely overestimated by GHRM. 


\section{Summary and conclusion}

In the preceding work we have investigated a number of aspects concerning Standard Model calculations of low-energy observables of which, using current phenomenological knowledge of nucleon structure functions, we have been able to determine to new levels of precision. The study of these structure functions both experimentally and theoretically will continue to enhance our understanding of the nucleon's internal structure.

Motivating much of the discussion in this work is the $\square_{\gamma Z}$ radiative correction to the weak charge of the proton. As the $Q_{\text {weak }}$ experiment is now in its analysis stage, it is essential to have a good understanding of all radiative corrections to $Q_{W}^{p}$. Utilising the Adelaide-Jefferson Lab-Manitoba model, we have calculated the energy dependence of $\square_{\gamma Z}^{V}$ up to energies relevant to the proposed MOLLER experiment. The proton and deuteron inelastic asymmetries were also determined.

After reviewing previous efforts in Chapter 4, most of Chapter 5 was devoted to the construction of the AJM model's interference structure functions. Starting with the division of the dispersion integral into different kinematic regions depending on the physics involved, we transformed the electromagnetic structure functions into their interference analogues. By matching the structure functions at the boundaries between the regions, we were able to constrain the interference cross sections using parton distribution functions. Furthermore, in using the quadrature method used to combine errors, we followed the standard conventions in the literature and had a consistent way of dealing with any uncertainties which arose in the analysis. Using the same method to construct deuteron structure functions, we tested the AJM model predictions of the parity-violating inelastic asymmetries by comparing with empirical data from electron-deuteron scattering. Within our quoted errors, those results were in good agreement with the experimental data, confirming the reliability of the model.

Having constructed a robust model of the interference structure functions, we proceeded to calculate the $\square_{\gamma Z}^{V}$ correction in Chapter 6. Since this correction is important in both the $Q_{\text {weak }}$ measurement of the proton's weak charge and the MOLLER experiment, the initial low-energy calculation was extended upto $12 \mathrm{GeV}$. In both cases, the AJM model's determination of 
$\square_{\gamma Z}^{V}$ gave uncertainties well within the error budget. In fact, at $Q_{\text {weak }}$ energies, the $\gamma Z$ box was calculated to a precision more than twice that of previous best estimates. For the MOLLER experiment, the relative contributions to the $\square_{\gamma Z}^{V}$ correction from the various kinematic regions differed significantly from the low-energy evaluation. In particular, the Regge region became the dominant part of the total correction. In order to ensure that any model dependence from this region was properly accounted for, we used a number of physically different models to compare with the AJM determination. Although the majority of these models were in good agreement, an additional error needed to be included to account for this model dependence. Nevertheless the final uncertainty remained within the experimental budget. The more recent E08-011 results for the parity-violating deep inelastic scattering asymmetry were also studied in this chapter, with the theory values again in matching well with the data.

Although we have examined the energy dependence of the $\gamma Z$ box correction, both the $Q_{\text {weak }}$ and the MOLLER experiment operate at finite $Q^{2}$. Since the framework used to calculate $\square_{\gamma Z}^{V}$ is the dispersion formalism, which has been used in the form derived at zero momentum transfer, the additional $Q^{2}$ dependence needs to be taken into account. Future efforts in this area would prove valuable. Additional experiments to measure the $\gamma Z$ structure functions would also help to further lower the current uncertainty on the $\square_{\gamma Z}$ correction.

While this work has given considerable attention to the $\gamma Z$ box correction, the structure functions developed in the AJM model may also be used to study other low energy phenomena. In Chapter 7, we used the electromagnetic parametrisations of the cross sections as inputs in the generalised Baldin sum rule, to determined the momentum transfer dependence of the electric and magnetic polarisabilities. Our results significantly improved upon the earlier findings of Sirbitsev and Bluden, showing good convergence towards the real photon point. An extrapolation of our results yielded a value for the static electromagnetic polarisabilities which was in excellent agreement with previous determinations. We were also able to determine the 'radius' of these polarisabilities, making contact with estimates from heavy baryon chiral perturbation theory.

In the future, this analysis would benefit from increased electron-nucleon cross section data in the very low $Q^{2}$ region $\left(Q^{2}<0.06 \mathrm{GeV}^{2}\right)$, since this would remove the need to extrapolate to the photoproduction point. Additionally, such data would reduce the uncertainties currently present in the resonance region parametrisation, resulting in smaller errors on the final estimates of both the $Q^{2}$-evolution of the polarisablities and their static values. To further enhance our understanding of the internal structure of the nucleon, such efforts are invaluable.

Finally, by studying the moments of the electromagnetic and interference structure functions we showed that duality holds at an unexpected level of precision to reasonably low momentum transfer, $Q^{2} \sim 1 \mathrm{GeV}^{2}$. Comparing the (proton and neutron) second moments of the total electromagnetic $F_{2}$ struc- 
ture function with the leading-twist moments, the close agreement between the two provided evidence of small higher-twist contributions. While there was marginally greater variation between the leading-twist and total structure function moments in the $\gamma Z$ moments, the uncertainties in the interference parametrisations were also larger and the results remained consistent with the $\gamma \gamma$ moments. The confirmation of duality further highlights the reliability of the AJM model: since the higher-twist contributions are small, the averaged $\gamma Z$ structure functions are constrained to resemble the leading-twist structure functions, preventing the uncertainty from becoming overly large.

An obvious extension to this analysis involves extracting the nucleon matrix elements from the higher-twist contributions. With the accuracy of the AJM model structure functions, we are in a position to calculate these matrix elements to a better precision than previous estimates [196, 197]. It would also be interesting to compare the higher-twist results found using the AJM model with those determined using lattice QCD [198] and other phenomenological models $[199,200]$. 



\section{Further determinations of} $\Re e \square_{\gamma Z}^{V}$

While the $\gamma Z$ box correction has been calculated at $Q_{\text {weak }}$ and MOLLER energies in the main body of this thesis, in order to fit to the $E=0$ point, data from other PVES experiments is also needed [89]. However, the $\square_{\gamma Z}$ correction must be included in each of these data points. In the following table we show the results for $\Re e \square_{\gamma Z}^{V}$ at the kinematics of these additional measurements, most of which were originally tabulated in Ref. [201].

Table A.1: The $\Re e \square_{\gamma Z}^{V}$ correction evaluated for additional parity-violating elastic scattering experiments.

\begin{tabular}{llcccc}
\hline \hline Collaboration & $Q^{2}\left(\mathrm{GeV}^{2}\right)$ & $\theta$ & $E(\mathrm{GeV})$ & $\Re e \square_{\gamma Z}^{V}\left(\times 10^{-3}\right)$ & Ref. \\
\hline PVA4 & 0.23 & 35.3 & 0.85 & $4.61 \pm 0.35$ & {$[88]$} \\
PVA4 & 0.108 & 35.4 & 0.57 & $3.75 \pm 0.29$ & {$[202]$} \\
HAPPEX & 0.477 & 12.3 & 3.35 & $7.81 \pm 0.80$ & {$[203]$} \\
HAPPEX & 0.099 & 6.0 & 3.03 & $7.55 \pm 0.76$ & {$[204]$} \\
G0 & 0.122 & 6.68 & 3.03 & $7.55 \pm 0.76$ & {$[205]$} \\
G0 & 0.128 & 6.84 & 3.03 & $7.55 \pm 0.76$ & {$[205]$} \\
G0 & 0.136 & 7.06 & 3.03 & $7.55 \pm 0.76$ & {$[205]$} \\
G0 & 0.144 & 7.27 & 3.03 & $7.55 \pm 0.76$ & {$[205]$} \\
G0 & 0.153 & 7.5 & 3.03 & $7.55 \pm 0.76$ & {$[205]$} \\
G0 & 0.164 & 7.77 & 3.03 & $7.55 \pm 0.76$ & {$[205]$} \\
G0 & 0.177 & 8.09 & 3.03 & $7.55 \pm 0.76$ & {$[205]$} \\
G0 & 0.192 & 8.43 & 3.03 & $7.55 \pm 0.76$ & {$[205]$} \\
G0 & 0.21 & 8.84 & 3.03 & $7.55 \pm 0.76$ & {$[205]$} \\
G0 & 0.232 & 9.31 & 3.03 & $7.55 \pm 0.76$ & {$[205]$} \\
G0 & 0.262 & 9.92 & 3.03 & $7.55 \pm 0.76$ & {$[205]$} \\
G0 & 0.299 & 10.63 & 3.03 & $7.55 \pm 0.76$ & {$[205]$} \\
G0 & 0.344 & 11.45 & 3.03 & $7.55 \pm 0.76$ & {$[205]$} \\
G0 & 0.41 & 12.59 & 3.03 & $7.55 \pm 0.76$ & {$[205]$} \\
\hline \hline
\end{tabular}


A. Further determinations of $\Re e \square_{\gamma Z}^{V}$

Table A.1 (continued): The $\Re e \square_{\gamma Z}^{V}$ correction evaluated for additional parityviolating elastic scattering experiments.

\begin{tabular}{llcccc}
\hline \hline Collaboration & $Q^{2}\left(\mathrm{GeV}^{2}\right)$ & $\theta$ & $E(\mathrm{GeV})$ & $\Re e \square_{\gamma Z}^{V}\left(\times 10^{-3}\right)$ & Ref. \\
\hline G0 & 0.511 & 14.2 & 3.03 & $7.55 \pm 0.76$ & {$[205]$} \\
G0 & 0.631 & 15.98 & 3.03 & $7.55 \pm 0.76$ & {$[205]$} \\
G0 & 0.788 & 18.16 & 3.03 & $7.55 \pm 0.76$ & {$[205]$} \\
G0 & 0.997 & 20.9 & 3.03 & $7.55 \pm 0.76$ & {$[205]$} \\
HAPPEX & 0.109 & 6.0 & 3.18 & $7.68 \pm 0.78$ & {$[206]$} \\
HAPPEX & 0.624 & 13.7 & 3.48 & $7.91 \pm 0.82$ & {$[207]$} \\
\hline \hline
\end{tabular}




\section{List of publications}

"Momentum transfer dependence of the proton's electric and magnetic polarizabilities"

N. L. Hall, A. W. Thomas and R. D. Young

Phys. Rev. D 89, 117502 (2014) [arXiv:nucl-th/1401.8062].

"Hadronic gamma-Z box corrections in Møller scattering"

N. L. Hall, P. G. Blunden, W. Melnitchouk, A. W. Thomas and R. D. Young Phys. Lett. B 731, 287 (2014) [arXiv:nucl-th/1311.3389].

"Constrained gamma-Z interference corrections to parity-violating electron scattering"

N. L. Hall, P. G. Blunden, W. Melnitchouk, A. W. Thomas and R. D. Young Phys. Rev. D 88, 013011 (2013) [arXiv:nucl-th/1304.7877].

"Constrained gamma-Z interference corrections to parity-violating electron scattering"

N. L. Hall, P. G. Blunden, W. Melnitchouk, A. W. Thomas and R. D. Young contributed to Proceedings of Workshop on Polarized Electron Beams, MIT. Boston, MA, March 2013. 



\section{Bibliography}

[1] G. Aad et al. (ATLAS Collaboration), Phys. Lett. B716, 1 (2012).

[2] S. Chatrchyan et al. (CMS Collaboration), Phys. Lett. B716, 30 (2012).

[3] J. Beringer et al. (Particle Data Group), Phys. Rev. D86, 010001 (2012).

[4] S. Glashow, Nucl. Phys. 22, 579 (1961).

[5] P. Langacker, The standard model and beyond (CRC Press, 2010).

[6] A. Salam, Conf. Proc. C680519, 367 (1968).

[7] S. Weinberg, Phys. Rev. Lett. 19, 1264 (1967).

[8] G. Guralnik, C. Hagen, and T. Kibble, Phys. Rev. Lett. 13, 585 (1964).

[9] F. Englert and R. Brout, Phys. Rev. Lett. 13, 321 (1964).

[10] P. W. Higgs, Phys. Rev. Lett. 13, 508 (1964).

[11] P. W. Higgs, Phys. Lett. 12, 132 (1964).

[12] G. 't Hooft, Nucl. Phys. B35, 167 (1971).

[13] G. 't Hooft and M. Veltman, Nucl.Phys. B44, 189 (1972).

[14] G. 't Hooft and M. Veltman, Nucl.Phys. B50, 318 (1972).

[15] T. Lee and C.-N. Yang, Phys. Rev. 104, 254 (1956).

[16] C. Wu, E. Ambler, R. Hayward, D. Hoppes, and R. Hudson, Phys. Rev. 105, 1413 (1957).

[17] R. Garwin, L. Lederman, and M. Weinrich, Phys. Rev. 105, 1415 (1957).

[18] P. Baird, S. Brimicombe, R. Hunt, G. Roberts, P. Sandars, et al., Phys. Rev. Lett. 39, 798 (1977).

[19] L. Barkov and M. Zolotorev, JETP Lett. 27, 357 (1978).

[20] L. Lewis, J. Hollister, D. Soreide, E. Lindahl, and E. Fortson, Phys. Rev. Lett. 39, 795 (1977). 
[21] C. Prescott, W. Atwood, R. L. Cottrell, H. DeStaebler, E. L. Garwin, et al., Phys. Lett. B77, 347 (1978).

[22] C. Prescott, W. Atwood, R. L. Cottrell, H. DeStaebler, E. L. Garwin, et al., Phys. Lett. B84, 524 (1979).

[23] F. Hasert et al. (Gargamelle Neutrino Collaboration), Phys.Lett. B46, 138 (1973).

[24] F. Hasert, H. Faissner, W. Krenz, J. Von Krogh, D. Lanske, et al., Phys.Lett. B46, 121 (1973).

[25] F. Hasert et al. (Gargamelle Neutrino Collaboration), Nucl.Phys. B73, 1 (1974).

[26] G. Arnison et al. (UA1 Collaboration), Phys. Lett. B126, 398 (1983).

[27] P. Bagnaia et al. (UA2 Collaboration), Phys. Lett. B129, 130 (1983).

[28] K. Kumar, S. Mantry, W. Marciano, and P. Souder, Ann. Rev. Nucl. Part. Sci. 63, 237 (2013).

[29] G. Zeller et al. (NuTeV Collaboration), Phys. Rev. Lett. 88, 091802 (2002).

[30] W. Bentz, I. Cloet, J. Londergan, and A. Thomas, Phys. Lett. B693, $462(2010)$.

[31] S. J. Brodsky, I. Schmidt, and J.-J. Yang, Phys. Rev. D70, 116003 (2004).

[32] K.-P. Diener, S. Dittmaier, and W. Hollik, Phys. Rev. D72, 093002 (2005).

[33] M. Hirai, S. Kumano, and T.-H. Nagai, Phys. Rev. D71, 113007 (2005).

[34] J. Erler and M. J. Ramsey-Musolf, Phys. Rev. D72, 073003 (2005).

[35] T. Aoyama, M. Hayakawa, T. Kinoshita, and M. Nio, Phys. Rev. Lett. 109, 111808 (2012).

[36] G. Jungman, M. Kamionkowski, and K. Griest, Phys. Rept. 267, 195 (1996).

[37] F. Quevedo, S. Krippendorf, and O. Schlotterer (2010).

[38] K. Hirata et al. (Kamiokande-II Collaboration), Phys. Lett. B280, 146 (1992).

[39] Q. Ahmad et al. (SNO Collaboration), Phys. Rev. Lett. 89, 011301 (2002). 
[40] J. Davis, Raymond, D. S. Harmer, and K. C. Hoffman, Phys. Rev. Lett. 20, 1205 (1968).

[41] T. Yanagida, Conf. Proc. C7902131, 95 (1979).

[42] M. Gell-Mann, P. Ramond, and R. Slansky, Conf. Proc. C790927, 315 (1979).

[43] P. Minkowski, Phys. Lett. B67, 421 (1977).

[44] W. de Boer and C. Sander, Phys. Lett. B585, 276 (2004).

[45] J. Wess and B. Zumino, Nucl. Phys. B70, 39 (1974).

[46] R. Haag, J. T. Lopuszanski, and M. Sohnius, Nucl. Phys. B88, 257 (1975).

[47] S. R. Coleman and J. Mandula, Phys. Rev. 159, 1251 (1967).

[48] T. Kaluza, Sitzungsber.Preuss.Akad.Wiss.Berlin (Math.Phys.) 1921, 966 (1921).

[49] O. Klein, Z.Phys. 37, 895 (1926).

[50] M. Stoye (CMS Collaboration), Nuovo Cim. C035, 333 (2012).

[51] D. Teyssier (ATLAS and CMS Collaborations) (2014).

[52] G. Azuelos, P. Depommier, R. Mazini, and K. Strahl (1999).

[53] G. Aad et al. (ATLAS Collaboration) (2014).

[54] D. Armstrong, A. Asaturyan, T. Averett, J. Benesch, J. Birchall, et al. (2012).

[55] J. Mammei (MOLLER Collaboration), Nuovo Cim. C035N04, 203 (2012).

[56] D. Wang et al. (Jefferson Lab Hall A Collaboration), Phys. Rev. Lett. 111, 082501 (2013).

[57] P. Souder (H1 and ZEUS Collaborations), AIP Conf. Proc. 1441, 123 (2012).

[58] R. Pohl, A. Antognini, F. Nez, F. D. Amaro, F. Biraben, et al., Nature 466, 213 (2010).

[59] F. An et al. (Daya Bay Collaboration) (2014).

[60] M. Kobayashi and T. Maskawa, Prog. Theor. Phys. 49, 652 (1973).

[61] N. Cabibbo, Phys. Rev. Lett. 10, 531 (1963). 
[62] K. Ikado et al. (Belle Collaboration), Phys.Rev.Lett. 97, 251802 (2006).

[63] B. Aubert et al. (BaBar Collaboration), Phys.Rev.Lett. 87, 091801 (2001).

[64] R. Aaij et al. (LHCb collaboration), Phys.Lett. B726, 151 (2013).

[65] D. Clowe, M. Bradac, A. H. Gonzalez, M. Markevitch, S. W. Randall, et al., Astrophys.J. 648, L109 (2006).

[66] A. Aab et al. (Pierre Auger Collaboration), Astrophys.J. 789, L34 (2014).

[67] A. Aab et al. (Pierre Auger Collaboration), Astrophys.J. 789, 160 (2014).

[68] J. Erler, J. Phys. Conf. Ser. 485, 012010 (2014).

[69] T. E. W. Group (CDF Collaboration, D0 Collaboration) (2009).

[70] T. E. W. Group (CDF Collaboration, D0 Collaboration) (2009).

[71] G. Bennett et al. (Muon G-2 Collaboration), Phys.Rev. D73, 072003 (2006).

[72] D. Webber et al. (MuLan Collaboration), Phys.Rev.Lett. 106, 041803 (2011).

[73] M. Musolf, T. Donnelly, J. Dubach, S. Pollock, S. Kowalski, et al., Phys. Rept. 239, 1 (1994).

[74] J. Erler and S. Su, Prog. Part. Nucl. Phys. 71, 119 (2013).

[75] A. Sirlin and A. Ferroglia, Rev. Mod. Phys. 85 (2013).

[76] M. Bouchiat, J. Guena, L. Hunter, and L. Pottier, Phys. Lett. B117, 358 (1982).

[77] P. Bucksbaum, E. Commins, and L. Hunter, Phys. Rev. Lett. 46, 640 (1981).

[78] R. Conti, P. Bucksbaum, S. Chu, E. Commins, and L. Hunter, Phys. Rev. Lett. 42, 343 (1979).

[79] C. Wood, S. Bennett, D. Cho, B. Masterson, J. Roberts, et al., Science 275, 1759 (1997).

[80] S. Bennett and C. E. Wieman, Phys. Rev. Lett. 82, 2484 (1999).

[81] S. Bennett and C. Wieman, Phys. Rev. Lett. 82, 4153 (1999).

[82] V. Dzuba, J. Berengut, V. Flambaum, and B. Roberts, Phys.Rev.Lett. 109, 203003 (2012). 
[83] S. Porsev, K. Beloy, and A. Derevianko, Phys.Rev. D82, 036008 (2010).

[84] P. Anthony et al. (SLAC E158 Collaboration), Phys. Rev. Lett. 95, 081601 (2005).

[85] K. Aniol et al. (HAPPEX Collaboration), Phys.Rev.Lett. 82, 1096 (1999).

[86] T. Ito et al. (SAMPLE Collaboration), Phys.Rev.Lett. 92, 102003 (2004).

[87] D. Spayde et al. (SAMPLE Collaboration), Phys.Lett. B583, 79 (2004).

[88] F. Maas et al. (A4 Collaboration), Phys.Rev.Lett. 93, 022002 (2004).

[89] D. Androic et al. (Qweak Collaboration), Phys. Rev. Lett. 111, 141803 (2013).

[90] N. L. Hall, P. G. Blunden, W. Melnitchouk, A. W. Thomas, and R. Young, Phys. Rev. D88, 013011 (2013).

[91] D. Androic et al. (G0 Collaboration) (2012).

[92] R. D. Young, R. D. Carlini, A. W. Thomas, and J. Roche, Phys. Rev. Lett. 99, 122003 (2007).

[93] D. Wang et al. (PVDIS Collaboration), Nature 506, 67 (2014).

[94] K. Kumar, private communication (2013).

[95] J. Benesch et al., The MOLLER Experiment: Measurement Of a Lepton Lepton Electroweak Reaction (2011).

[96] W. Marciano and A. Sirlin, Phys. Rev. D27, 552 (1983).

[97] W. Marciano and A. Sirlin, Phys. Rev. D29, 75 (1984).

[98] J. Erler, A. Kurylov, and M. J. Ramsey-Musolf, Phys. Rev. D68, 016006 (2003).

[99] M. Musolf and B. R. Holstein, Phys. Lett. B242, 461 (1990).

[100] J. Arrington, P. Blunden, and W. Melnitchouk, Prog. Part. Nucl. Phys. 66, 782 (2011).

[101] P. Blunden, W. Melnitchouk, and A. Thomas, Phys. Rev. Lett. 107, 081801 (2011).

[102] P. Blunden, W. Melnitchouk, and A. Thomas, Phys. Rev. Lett. 109, 262301 (2012).

[103] P. Blunden, private communication (2013). 
[104] N. L. Hall, P. G. Blunden, W. Melnitchouk, A. W. Thomas, and R. Young, Phys. Lett. B731, 287 (2014).

[105] H. Q. Zhou, C. W. Kao, and S. N. Yang, Phys. Rev. Lett. 99, 262001 (2007).

[106] J. Tjon, P. Blunden, and W. Melnitchouk, Phys. Rev. C79, 055201 (2009).

[107] M. Gorchtein and C. Horowitz, Phys. Rev. Lett. 102, 091806 (2009).

[108] A. Sibirtsev, P. Blunden, W. Melnitchouk, and A. Thomas, Phys. Rev. D82, 013011 (2010).

[109] B. C. Rislow and C. E. Carlson, Phys. Rev. D83, 113007 (2011).

[110] M. Gorchtein, C. Horowitz, and M. J. Ramsey-Musolf, Phys. Rev. C84, 015502 (2011).

[111] M. E. Peskin and D. V. Schroeder, An introduction to quantum field theory (Westview Press, 1995).

[112] Y. Liang et al. (2013), arXiv:nucl-ex/0410027v2.

[113] Y. Liang et al. (2004), arXiv:nucl-ex/0410027v1.

[114] F. Aaron et al. (H1 Collaboration), JHEP 1209, 061 (2012).

[115] J. I. Friedman and H. W. Kendall, Ann. Rev. Nucl. Part. Sci. 22, 203 (1972).

[116] E. D. Bloom, D. Coward, H. DeStaebler, J. Drees, G. Miller, et al., Phys. Rev. Lett. 23, 930 (1969).

[117] S. Bethke, Nucl. Phys. Proc. Suppl. 121, 74 (2003).

[118] R. P. Feynman, Phys. Rev. Lett. 23, 1415 (1969).

[119] J. Callan, Curtis G. and D. J. Gross, Phys.Rev.Lett. 22, 156 (1969).

[120] A. Martin, W. Stirling, R. Thorne, and G. Watt, Eur. Phys. J. C63, 189 (2009).

[121] M. Christy and P. E. Bosted, Phys. Rev. C81, 055213 (2010).

[122] P. Bosted and M. Christy, Phys. Rev. C77, 065206 (2008).

[123] C. E. Carlson and B. C. Rislow, Phys. Rev. D85, 073002 (2012).

[124] N. Nikolaev and B. G. Zakharov, Z. Phys. C53, 331 (1992).

[125] N. N. Nikolaev and B. Zakharov, Z. Phys. C49, 607 (1991). 
[126] A. H. Mueller, Nucl. Phys. B415, 373 (1994).

[127] G. Cvetic, D. Schildknecht, B. Surrow, and M. Tentyukov, Eur. Phys. J. C20, 77 (2001).

[128] J. R. Forshaw, R. Sandapen, and G. Shaw, JHEP 0611, 025 (2006).

[129] G. Cvetic, D. Schildknecht, and A. Shoshi, Eur. Phys. J. C13, 301 (2000).

[130] L. Lipatov, Phys. Rept. 286, 131 (1997).

[131] P. Collins, An introduction to Regge theory and high-energy Physics (Cambridge University Press, 1977).

[132] A. Donnachie and P. Landshoff, Phys. Lett. B296, 227 (1992).

[133] A. Capella, A. Kaidalov, C. Merino, and J. Tran Thanh Van, Phys. Lett. B337, 358 (1994).

[134] J. Alwall and G. Ingelman, Phys. Lett. B596, 77 (2004).

[135] J. Sakurai and D. Schildknecht, Phys. Lett. B40, 121 (1972).

[136] K. Nakamura et al. (Particle Data Group), J. Phys. G37, 075021 (2010).

[137] A. Martin, R. Roberts, W. Stirling, and R. Thorne, Eur. Phys. J. C28, 455 (2003).

[138] L. Tiator, D. Drechsel, S. Kamalov, and M. Vanderhaeghen, Eur. Phys. J. ST 198, 141 (2011).

[139] H.-L. Lai, M. Guzzi, J. Huston, Z. Li, P. M. Nadolsky, et al., Phys. Rev. D82, 074024 (2010).

[140] B. C. Rislow and C. E. Carlson, Phys.Rev. D88, 013018 (2013).

[141] S. Alekhin, J. Blumlein, and S. Moch, Phys. Rev. D86, 054009 (2012).

[142] A. Accardi, W. Melnitchouk, J. Owens, M. Christy, C. Keppel, et al., Phys. Rev. D84, 014008 (2011).

[143] J. Owens, A. Accardi, and W. Melnitchouk, Phys. Rev. D87, 094012 (2013).

[144] R. D. Ball, L. Del Debbio, S. Forte, A. Guffanti, J. I. Latorre, et al., Nucl. Phys. B838, 136 (2010).

[145] P. Jimenez-Delgado and E. Reya, Phys. Rev. D80, 114011 (2009).

[146] M. Gorchtein, private communication (2012). 
[147] S. Alekhin, private communication (2012).

[148] J. Breitweg et al. (ZEUS Collaboration), Phys. Lett. B487, 273 (2000).

[149] O. Lalakulich and E. A. Paschos, Phys. Rev. D71, 074003 (2005).

[150] O. Lalakulich, E. A. Paschos, and G. Piranishvili, Phys. Rev. D74, 014009 (2006).

[151] O. Lalakulich, W. Melnitchouk, and E. Paschos, Phys. Rev. C75, 015202 (2007).

[152] N. C. Mukhopadhyay, M. Ramsey-Musolf, S. J. Pollock, J. Liu, and H. Hammer, Nucl. Phys. A633, 481 (1998).

[153] R. Carlini, private communication (2012).

[154] X. Zheng, private communication (2013).

[155] E. Derman and W. J. Marciano, Annals Phys. 121, 147 (1979).

[156] A. Czarnecki and W. J. Marciano, Phys. Rev. D53, 1066 (1996).

[157] A. Denner and S. Pozzorini, Eur. Phys. J. C7, 185 (1999).

[158] F. J. Petriello, Phys. Rev. D67, 033006 (2003).

[159] M. Kuroda and D. Schildknecht, Phys. Rev. D85, 094001 (2012).

[160] L. Hall, N. W. Thomas, A. and D. Young, R. Phys. Rev. D 89, 117502 (2014).

[161] H. Griesshammer, J. McGovern, D. Phillips, and G. Feldman, Prog. Part. Nucl. Phys. 67, 841 (2012).

[162] Y. Liang, M. E. Christy, R. Ent, and C. E. Keppel, Phys. Rev. C73, 065201 (2006).

[163] S. Drell and A. C. Hearn, Phys. Rev. Lett. 16, 908 (1966).

[164] S. Gerasimov, Sov. J. Nucl. Phys. 2, 430 (1966).

[165] A. M. Baldin, Nucl. Phys. 18, 310 (1960).

[166] L. I. Lapidus, Sov. Phys. JETP 16, 964 (1963).

[167] D. Drechsel, B. Pasquini, and M. Vanderhaeghen, Phys. Rept. 378, 99 (2003).

[168] J. Roche et al. (VCS and A1 Collaborations), Phys. Rev. Lett. 85, 708 (2000). 
[169] P. Bourgeois, Y. Sato, J. Shaw, R. Alarcon, A. Bernstein, et al., Phys. Rev. Lett. 97, 212001 (2006).

[170] A. Sibirtsev and P. Blunden, Phys. Rev. C88, 065202 (2013).

[171] V. Lensky and J. A. McGovern (2014).

[172] L. Whitlow, S. Rock, A. Bodek, E. Riordan, and S. Dasu, Phys. Lett. B250, 193 (1990).

[173] D. Babusci, G. Giordano, and G. Matone, Phys. Rev. C57, 291 (1998).

[174] V. Olmos de Leon, F. Wissmann, P. Achenbach, J. Ahrens, H. Arends, et al., Eur. Phys. J. A10, 207 (2001).

[175] T. R. Hemmert, B. R. Holstein, G. Knochlein, and D. Drechsel, Phys. Rev. D62, 014013 (2000).

[176] E. D. Bloom and F. J. Gilman, Phys. Rev. D4, 2901 (1971).

[177] E. D. Bloom and F. J. Gilman, Phys. Rev. Lett. 25, 1140 (1970).

[178] A. De Rujula, H. Georgi, and H. D. Politzer, Annals Phys. 103, 315 (1977).

[179] A. De Rujula, H. Georgi, and H. D. Politzer, Phys.Lett. B64, 428 (1977).

[180] W. Melnitchouk, R. Ent, and C. Keppel, Phys. Rept. 406, 127 (2005).

[181] I. Niculescu, C. Armstrong, J. Arrington, K. Assamagan, O. Baker, et al., Phys. Rev. Lett. 85, 1186 (2000).

[182] Y. Liang. Ph.D. Thesis. PhD thesis, The American University (2003).

[183] I. Niculescu, C. Armstrong, J. Arrington, K. Assamagan, O. Baker, et al., Phys. Rev. Lett. 85, 1182 (2000).

[184] A. Airapetian et al. (HERMES Collaboration), Phys.Rev.Lett. 90, 092002 (2003).

[185] F. R. Wesselmann et al. (RSS Collaboration), Phys.Rev.Lett. 98, 132003 (2007).

[186] J. Arrington, R. Ent, C. Keppel, J. Mammei, and I. Niculescu, Phys. Rev. C73, 035205 (2006).

[187] R. A. Brandt and G. Preparata, Nucl. Phys. B27, 541 (1972).

[188] O. Nachtmann, Nucl. Phys. B63, 237 (1973).

[189] K. G. Wilson, Phys. Rev. 179, 1499 (1969). 
[190] E. V. Shuryak and A. Vainshtein, Nucl. Phys. B199, 451 (1982).

[191] J. M. Cornwall and R. E. Norton, Phys. Rev. 177, 2584 (1969).

[192] S. Simula, Phys. Lett. B574, 189 (2003).

[193] F. Steffens and W. Melnitchouk, Phys. Rev. C73, 055202 (2006).

[194] A. W. Thomas and W. Weise, The structure of the nucleon (Wiley-VCH, 2001).

[195] J. Kelly, Phys. Rev. C70, 068202 (2004).

[196] R. Jaffe and M. Soldate, Phys.Lett. B105, 467 (1981).

[197] S. H. Lee, Phys.Rev. D49, 2242 (1994).

[198] M. Gockeler, R. Horsley, B. Klaus, D. Pleiter, P. E. Rakow, et al., Nucl.Phys. B623, 287 (2002).

[199] A. Signal, Nucl.Phys. B497, 415 (1997).

[200] A. Signal, Austral.J.Phys. 50, 61 (1997).

[201] R. D. Young, J. Roche, R. D. Carlini, and A. W. Thomas, Phys.Rev.Lett. 97, $102002(2006)$.

[202] F. Maas, K. Aulenbacher, S. Baunack, L. Capozza, J. Diefenbach, et al., Phys.Rev.Lett. 94, 152001 (2005).

[203] K. Aniol et al. (HAPPEX Collaboration), Phys.Rev. C69, 065501 (2004).

[204] K. Aniol et al. (HAPPEX Collaboration), Phys.Lett. B635, 275 (2006).

[205] D. Armstrong et al. (G0 Collaboration), Phys.Rev.Lett. 95, 092001 (2005).

[206] A. Acha et al. (HAPPEX collaboration), Phys.Rev.Lett. 98, 032301 (2007).

[207] Z. Ahmed et al. (HAPPEX collaboration), Phys.Rev.Lett. 108, 102001 (2012). 